\title{
Uniqueness and pseudolocality theorems of the mean curvature flow
}

\author{
Bing-Long Chen And Le Yin
}

\begin{abstract}
Mean curvature flow evolves isometrically immersed base manifolds $M$ in the direction of their mean curvatures in an ambient manifold $\bar{M}$. If the base manifold $M$ is compact, the short-time existence and uniqueness of the mean curvature flow are well known. For complete isometrically immersed submanifolds of arbitrary codimensions, the existence and uniqueness are still unsettled even in the Euclidean space. In this paper, we solve the uniqueness problem affirmatively for the mean curvature flow of general codimensions and general ambient manifolds. In the second part of the paper, inspired by the Ricci flow, we prove a pseudolocality theorem of mean curvature flow. As a consequence, we obtain a strong uniqueness theorem, which removes the assumption on the boundedness of the second fundamental form of the solution.
\end{abstract}

\section{Introduction}

Let $\left(\bar{M}^{\bar{n}}, \bar{g}\right)$ be a complete Riemannian (compact or noncompact) manifold, and $X_{0}:\left(M^{n}, g\right) \rightarrow \bar{M}^{\bar{n}}$ be an isometrically immersed Riemannian manifold. For any fixed point $x_{0} \in M^{n}, X, Y \in T_{x_{0}} M^{n}$, the second fundamental form II at $x_{0}$ is defined by $\operatorname{II}(X, Y)=\bar{\nabla}_{\tilde{X}} \tilde{Y}-\nabla_{\tilde{X}} \tilde{Y}=\left(\bar{\nabla}_{\tilde{X}} \tilde{Y}\right)^{\perp}$, where $M^{n}$ is regarded as a submanifold of $\bar{M}$ locally by the isometry $X_{0}, \bar{\nabla}$ and $\nabla$ are the covariant derivatives of $\bar{g}$ and $g$, respectively, $\tilde{X}, \tilde{Y}$ are any smooth extensions of $X$ and $Y$ on $\bar{M}^{\bar{n}}$. In a local coordinate system $\left\{x^{1}, x^{2}, \ldots, x^{n}\right\}$ on $M^{n}$, denote the second fundamental form by $h_{i j}=I I\left(\left(\partial / \partial x^{i}\right),\left(\partial / \partial x^{j}\right)\right)$ and the mean curvature by $H=g^{i j} h_{i j}$. The mean curvature flow (MCF) is a deformation $X_{t}: M^{n} \rightarrow \bar{M}^{\bar{n}}$ of $X_{0}$ in the direction of the mean curvature $H$

$$
\frac{\partial}{\partial t} X(x, t)=H(x, t), \text { for } x \in M^{n} \text { and } t \geq 0,
$$

with $X(x, 0)=X_{0}(x)$, where $M^{n}$ is equipped with the induced metric from $X(\cdot, t): M^{n} \rightarrow \bar{M}^{\bar{n}}$ and $H(x, t)$ is the corresponding mean curvature. 
We can write (1.1) in another form

$$
\frac{\partial}{\partial t} X(x, t)=\triangle X(x, t), \text { for } x \in M^{n} \text { and } t \geq 0,
$$

where $\triangle X^{\alpha}(x, t)=g^{i j}(x, t)\left(\left(\partial^{2} X^{\alpha} / \partial x^{i} \partial x^{j}\right)-\Gamma_{i j}^{k}\left(\partial X^{\alpha} / \partial x^{k}\right)+\bar{\Gamma}_{\beta \gamma}^{\alpha}\left(\partial X^{\beta} /\right.\right.$ $\left.\left.\partial x^{i}\right)\left(\partial X^{\gamma} / \partial x^{j}\right)\right)$ is the harmonic map Laplacian from the manifold $\left(M^{n}, g_{i j}\right.$ $(\cdot, t))$ to $\left(\bar{M}^{\bar{n}}, \bar{g}\right)$, and $g_{i j}(\cdot, t)$ is the induced metric from the inclusion $\operatorname{map} X(\cdot, t)$.

Various weak solutions to the MCF have been studied in the past 30 years by many mathematicians with different approaches, e.g., Brakke solutions, the level set solutions, etc. The existence, uniqueness and nonuniqueness of weak solutions for Euclidean (non)smooth hypersurface have been extensively studied. In this paper, motivated by geometric applications, we consider the classical solutions in general ambient Riemannian manifolds.

When $M^{n}$ is compact, the MCF (1.1) has a unique short-time solution, since (1.2) is a (degenerate) quasi-linear parabolic equation. For codimensional one complete immersed local Lipschitz hypersurfaces in the Euclidean space, we refer the readers to see $[11,12]$. For submanifolds of arbitrary codimensions in a general ambient Riemannian manifold, the short-time existence and the uniqueness of (1.1) have not been established in the literature. In this paper, we deal with the uniqueness problem of the mean curvature flow and derive the pseudolocality estimate.

The first main theorem of this paper is the following.

Theorem 1.1. Let $\left(\bar{M}^{\bar{n}}, \bar{g}\right)$ be a complete Riemannian manifold of dimension $\bar{n}$ such that the curvature and its covariant derivatives up to order 2 are bounded and the injectivity radius is bounded from below by a positive constant, i.e., there are constants $\bar{C}$ and $\bar{\delta}$ such that

$$
|\overline{\mathrm{R}} \mathrm{m}|+|\bar{\nabla} \overline{\mathrm{R}} \mathrm{m}|+\left|\bar{\nabla}^{2} \overline{\mathrm{R}} \mathrm{m}\right|(x) \leq \bar{C}, \quad \operatorname{inj}\left(\bar{M}^{\bar{n}}, x\right)>\bar{\delta}>0,
$$

for all $x \in \bar{M}^{\bar{n}}$. Let $X_{0}: M^{n} \rightarrow \bar{M}^{\bar{n}}$ be an isometrically immersed Riemannian manifold with bounded second fundamental form in $\bar{M}^{\bar{n}}$. Suppose $X_{1}(x, t)$ and $X_{2}(x, t)$ are two solutions to the mean curvature flow (1.1) on $M^{n} \times[0, T]$ with the same $X_{0}$ as initial data and with bounded second fundamental forms on $[0, T]$. Then $X_{1}(x, t)=X_{2}(x, t)$ for all $(x, t) \in M^{n} \times[0, T]$.

We remark that the uniqueness of the Ricci flow has been established by Zhu and the first author in [5]. More precisely, it was proved in [5] that the solutions of the Ricci flow in the class of bounded curvature with the same 
initial data are unique. We refer the readers to see an interesting application of this uniqueness theorem to the theory of the Ricci flow with surgery in dimension 3 and $4[2,4,21]$. We hope this MCF uniqueness theorem will also play roles in the theory of the mean curvature flow with surgery.

Since the MCF is degenerate in tangent directions, it is not a strictly parabolic system. In order to apply the standard theory of strict parabolic equations, we use the De Turck trick [7]. The idea is to pull back the MCF through a family of diffeomorphisms of the base manifold $M^{n}$ generated by solving a harmonic map flow coupled with the MCF, this gives us the MCF in harmonic map flow gauge, which is a strict parabolic system. Then we apply the uniqueness of the strict parabolic system. The issue is not quite straight forward as it seems. Because before applying the uniqueness theorem of a strict parabolic system on a noncompact manifold, we encounter two analytic difficulties. The first one is that we need to establish a short-time existence for the harmonic map flow between complete manifolds. The second one is to get a priori estimates for the harmonic map flow so that after pulling back, the solutions to the strictly parabolic system still satisfy suitable smooth or growth conditions.

In the classical theory of the harmonic map flow, people usually would like to impose certain convexity conditions to ensure the existence (e.g., the negative curvature condition [13] or convex condition [8]). We observed that in [5] the condition of injectivity radius bounded from below by a positive constant ensures certain uniform (local) convexity and this is sufficient to give the short-time existence and a priori estimates for the harmonic map flow. Note that the MCF is a kind of harmonic map flow with varying base metrics. In order to deal with the a priori estimates for MCF and harmonic map flow coupled with MCF, we have to consider the general harmonic map flow. These estimates have been dealt with systematically in this paper (Sections 2, 3 and 4).

Note that the injectivity radius of a Riemannian manifold with bounded curvature may decay exponentially. In the Ricci flow case [5], since we only have the curvature bound, we need make more effort to overcome this difficulty.

The difference of Theorem 1.1 with [5] is between the extrinsic and intrinsic geometries. In the present case, instead of the metrics as in the Ricci flow, we consider the equation of the position function.

As a direct consequence of Theorem 1.1, we have

Corollary 1.2. Let $\left(\bar{M}^{\bar{n}}, \bar{g}\right)$ be assumed as in Theorem 1.1 and $X_{t}: M^{n} \rightarrow$ $\bar{M}^{\bar{n}}$ be a solution to the mean curvature flow (1.1) on $M^{n} \times[0, T]$ with 
bounded second fundamental forms on $[0, T]$, and with complete isometric immersed $X_{0}: M \rightarrow M$ initial data. Let $\bar{\sigma}$ be an isometry of $\left(\bar{M}^{\bar{n}}, \bar{g}\right)$ such that there is an isometry $\sigma$ of $\left(M^{n}, g\right)$ to itself satisfying

$$
\left(\bar{\sigma} \circ X_{0}\right)(x)=\left(X_{0} \circ \sigma\right)(x)
$$

for all $x \in M^{n}$. Then we have

$$
\left(\bar{\sigma} \circ X_{t}\right)(x)=\left(X_{t} \circ \sigma\right)(x)
$$

for all $(x, t) \in M^{n} \times[0, T]$. In particular, the isometry subgroup of $\left(M^{n}, g\right)$ induced by an isometry subgroup of $\left(\bar{M}^{\bar{n}}, \bar{g}\right)$ at initial time remains to be an isometry subgroup of $\left(M^{n}, g_{t}\right)$ for any $t \in[0, T]$.

From the PDE point of view, it is a natural condition in Theorem 1.1 to assume that the second fundamental form of the solution is bounded. In the last part of the paper, we try to remove this condition. We remark that in [6], Chou and Zhu had obtained the strong uniqueness of the curve shortening flow for the locally Lipschitz continuous properly embedded curve whose two ends are presentable as graphs over semi-infinite line. Our strong uniqueness theorem is the following.

Theorem 1.3. Let $\bar{M}$ be an $\bar{n}$-dimensional complete Riemannian manifold satisfying $\sum_{i=0}^{3}\left|\bar{\nabla}^{i} \overline{\mathrm{R}} \mathrm{m}\right| \leq c_{0}^{2}$ and $\operatorname{inj}(\bar{M}) \geq i_{0}>0$. Let $X_{0}: M \rightarrow \bar{M}$ be an $n$-dimensional isometrically properly embedded submanifold with bounded second fundamental form in $\bar{M}$. We assume $X_{0}(M)$ is uniform graphic with some radius $r>0$. Suppose $X_{1}(x, t)$ and $X_{2}(x, t)$ are two smooth solutions to the mean curvature flow (1.1) on $M \times\left[0, T_{0}\right]$ properly embedded in $\bar{M}$ with the same $X_{0}$ as initial data. Then there is $0<T_{1} \leq T_{0}$ such that $X_{1}(x, t)=X_{2}(x, t)$ for all $(x, t) \in M \times\left[0, T_{1}\right]$.

Here roughly speaking, uniform graphic with radius $r(>0)$ means that for any $x_{0} \in X_{0}(M), X_{0}(M) \cap B_{\bar{M}}\left(x_{0}, r\right)$ is a graph. We say a submanifold $M \subset \bar{M}$ is properly embedded in a ball $B_{\bar{M}}\left(x_{0}, r_{0}\right)$ if either $M$ is closed or $\partial M$ has distance $\geq r_{0}$ from $x_{0}$. A submanifold $M \subset \bar{M}$ is said to be properly embedded in (complete manifold) $\bar{M}$ if either $M$ is closed or there is an $x_{0} \in \bar{M}$ such that $M$ is properly embedded in $B_{\bar{M}}\left(x_{0}, r_{0}\right)$ for any $r_{0}>0$.

The strong uniqueness theorem was proved as a consequence of Theorem 1.1 and pseudolocality theorem.

The pseudolocality theorem says that the behavior of the solution at a point can be controlled by the initial data of nearby points, no matter the 
solution or initial data outside the neighborhood behaviors like. Precisely the following theorem is proved in this paper.

Theorem 1.4. Let $\bar{M}$ be an $\bar{n}$-dimensional manifold satisfying $\sum_{i=0}^{3}$ $\left|\bar{\nabla}^{i} \overline{\mathrm{R}} \mathrm{m}\right| \leq c_{0}^{2}$ and $\operatorname{inj}(\bar{M}) \geq i_{0}>0$. Then for every $\alpha>0$ there exist $\varepsilon>0$, $\delta>0$ depending only on the constants $\bar{n}, c_{0}$ and $i_{0}$ with the following property. Suppose we have a smooth solution to the mean curvature flow $M_{t} \subset \bar{M}$ properly embedded in $B_{\bar{M}}\left(x_{0}, r_{0}\right)$ for $t \in[0, T]$, where $0<T \leq \varepsilon^{2} r_{0}^{2}$, and assume that at time zero, $M_{0}$ is a local $\delta$-Lipschitz graph of radius $r_{0}$ at $x_{0} \in M$ with $r_{0} \leq\left(i_{0} / 2\right)$. Then we have an estimate of the second fundamental form

$$
|A|(x, t)^{2} \leq \frac{\alpha}{t}+\left(\varepsilon r_{0}\right)^{-2}
$$

on $B_{\bar{M}}\left(x_{0}, \varepsilon r_{0}\right) \cap M_{t}$, for any $t \in[0, T]$.

We refer the reader to see the precise definition of $\delta$-Lipschitz graph in Section 7. The third covariant derivative of the curvature is a technical assumption which could be improved, we assume it only for simplicity. For most of interesting cases, we have all covariant derivative bounds.

We remark that for codimension one uniformly local Lipschitz hypersurface in Euclidean space, the estimate was firstly derived by Ecker and Huisken [11,12]. For higher-codimension case, under an additional condition which assumes that the submanifold is compact, the estimate was proved by Wang [24]. In codimension, one case, the constant $\delta$ in Theorem 1.4 does not need to be small; however, in higher-codimension case, as noted by [24], the smallness assumption is necessary in view of the example of Lawson and Osserman [18]. The strategy of the proofs of $[11,12,24]$ is to find a suitable gradient function. The philosophy is that this gradient function will serve as the lower order quantity as in the Bernstein trick, and the second fundamental form is the higher order quantity, then apply the maximum principle. This method has some difficulties in higher-codimensional case in current stage, treating pure local estimates for noncompact submanifolds (see $[24])$.

Our approach is completely different. This approach can be regarded as an integral version of Bernstein trick. It is a mean curvature flow analog of the corresponding estimate in Ricci flow given by Perelman [20]. The idea of localizing monotonicity formulas (see [9], or formula (7.8) in Section 7 of the present paper) plays a crucial role in our argument. We refer the readers to see $[1,9,10,16]$ (Euclidean case) for relevant details and references. 
As a nontrivial corollary of Theorem 1.4, we have

Corollary 1.5. Let $\bar{M}$ be an $\bar{n}$-dimensional complete manifold satisfying $\sum_{i=0}^{3}\left|\bar{\nabla}^{i} \overline{\mathrm{R}} \mathrm{m}\right| \leq c_{0}^{2}$ and $\operatorname{inj}(\bar{M}) \geq i_{0}>0$. Let $X_{0}: M \rightarrow \bar{M}$ be an $n$ dimensional isometrically properly embedded submanifold with bounded second fundamental form $|A| \leq c_{0}$ in $\bar{M}$. We assume $M_{0}=X_{0}(M)$ is uniform graphic with some radius $r>0$. Suppose $X(x, t)$ is a smooth solution to the mean curvature flow (1.1) on $M \times\left[0, T_{0}\right]$ properly embedded in $\bar{M}$ with $X_{0}$ as initial data. Then there is $T_{1}>0$ depending upon $c_{0}, i_{0}, r$ and the dimension $\bar{n}$ such that

$$
|A|(x, t) \leq 2 c_{0}
$$

for all $x \in M, 0 \leq t \leq \min \left\{T_{0}, T_{1}\right\}$.

This paper is organized as follows. In Section 2, we derive the injectivity radius estimate of an immersed manifold and some preliminary estimates for a general harmonic map flow. In Section 3, the higher-derivative estimates for the MCF are derived. In Section 4, we study the harmonic map flow coupled with the MCF. In Section 5, we deal with the uniqueness theorem of the mean curvature De Turck flow (or MCF in harmonic map flow gauge). In Section 6, we prove the uniqueness Theorem 1.1 and Corollary 1.2. In Section 7, we establish the pseudolocality Theorems 1.4 and 1.5 and prove the strong uniqueness Theorem 1.3.

\section{Preliminary estimates}

In the first part of this section, we will derive the injectivity radius estimate for isometrically immersed manifold $M^{n}$.

Theorem 2.1. Let $\left(\bar{M}^{\bar{n}}, \bar{g}\right)$ be a complete Riemannian manifold of dimension $\bar{n}$ with bounded curvature and the injectivity radius is bounded from below by a positive constant, i.e., there are constants $\bar{C}$ and $\bar{\delta}$ such that

$$
|\overline{\mathrm{R}} \mathrm{m}|(x) \leq \bar{C} \quad \text { and } \quad \operatorname{inj}\left(\bar{M}^{\bar{n}}, x\right) \geq \bar{\delta}>0, \text { for all } x \in \bar{M}^{\bar{n}}
$$

Let $X: M^{n} \rightarrow \bar{M}^{\bar{n}}$ be a complete isometrically immersed manifold with bounded second fundamental form $\left|h_{i j}^{\alpha}\right| \leq C$ in $\bar{M}^{\bar{n}}$, then there is a positive constant $\delta=\delta(\bar{C}, \bar{\delta}, C, \bar{n})$ such that the injectivity radius of $M^{n}$ satisfies

$$
\operatorname{inj}\left(M^{n}, x\right) \geq \delta>0, \text { for all } x \in M^{n}
$$


Uniqueness and pseudolocality theorems of the mean curvature flow 441

Proof. Fix $x_{0} \in M^{n}$, let $\left\{y^{1}, y^{2}, \ldots, y^{\bar{n}}\right\}$ and $\left\{x^{1}, x^{2}, \ldots, x^{n}\right\}$ be any two local coordinates of $\bar{M}^{\bar{n}}$ and $M^{n}$ at $y_{0}\left(=X\left(x_{0}\right)\right)$ and $x_{0}$, respectively, recall that the second fundamental form can be written in these local coordinates in the following form

$$
\begin{aligned}
h_{i j}^{\alpha} & =\frac{\partial^{2} y^{\alpha}}{\partial x^{i} \partial x^{j}}-\Gamma_{i j}^{k} \frac{\partial y^{\alpha}}{\partial x^{k}}+\bar{\Gamma}_{\beta \gamma}^{\alpha} \frac{\partial y^{\beta}}{\partial x^{i}} \frac{\partial y^{\gamma}}{\partial x^{j}} \\
& =\nabla_{i} \nabla_{j}\left(y^{\alpha}\right)+\bar{\Gamma}_{\beta \gamma}^{\alpha} \frac{\partial y^{\beta}}{\partial x^{i}} \frac{\partial y^{\gamma}}{\partial x^{j}}, \text { for } \alpha=1,2, \ldots, \bar{n},
\end{aligned}
$$

where $\nabla_{i} \nabla_{j}\left(y^{\alpha}\right)$ is the Hessian of $y^{\alpha}$, which is viewed as a function of $M^{n}$ near $x_{0}$. In the following argument, we denote by $\bar{C}_{1}$ various constants depending only on $\bar{C}, C$ and $\bar{\delta}$.

Define $f(x)=\bar{d}^{2}\left(y_{0}, X(x)\right)$ on $M^{n} \cap X^{-1}\left(\bar{B}\left(y_{0}, \bar{C}_{1}\right)\right)$ for some $\bar{C}_{1} \leq \bar{\delta}$, then $\nabla_{j} f=\left(\partial f / \partial y^{\alpha}\right)\left(\partial y^{\alpha} / \partial x^{j}\right)$ and the Hessian of $f$ with respect to the metric $g$ on $M^{n} \cap X^{-1}\left(\bar{B}\left(y_{0}, \bar{C}_{1}\right)\right)$ can be computed as follows

$$
\begin{aligned}
\nabla_{i} \nabla_{j} f= & \frac{\partial}{\partial x^{i}} \nabla_{j} f-\Gamma_{i j}^{k} \nabla_{k} f \\
= & \left(\frac{\partial^{2} f}{\partial y^{\alpha} \partial y^{\beta}}-\bar{\Gamma}_{\alpha \beta}^{\gamma} \frac{\partial f}{\partial y^{\gamma}}\right) \frac{\partial y^{\alpha}}{\partial x^{j}} \frac{\partial y^{\beta}}{\partial x^{i}} \\
& +\frac{\partial f}{\partial y^{\alpha}}\left(\frac{\partial^{2} y^{\alpha}}{\partial x^{i} \partial x^{j}}-\Gamma_{i j}^{k} \frac{\partial y^{\alpha}}{\partial x^{k}}+\bar{\Gamma}_{\beta \gamma}^{\alpha} \frac{\partial y^{\beta}}{\partial x^{i}} \frac{\partial y^{\gamma}}{\partial x^{j}}\right) \\
= & \bar{\nabla}_{\alpha} \bar{\nabla}_{\beta} \bar{d}^{2} \frac{\partial y^{\alpha}}{\partial x^{j}} \frac{\partial y^{\beta}}{\partial x^{i}}+2 \bar{d} \bar{\nabla}_{\alpha} \bar{d} \cdot h_{i j}^{\alpha} .
\end{aligned}
$$

Using Hessian comparison theorem on $\bar{M}^{\bar{n}}$ and choosing $\bar{C}_{1}$ suitable small so that $\bar{d}$ is suitable small, we get

$$
\nabla_{i} \nabla_{j} f \geq \frac{1}{2} g_{i j}
$$

on $M^{n} \cap X^{-1}\left(\bar{B}\left(y_{0}, \bar{C}_{1}\right)\right)$. Now we claim that any closed geodesic starting and ending at $x_{0}$ on $\left(M^{n}, g\right)$ must have length $\geq 2 \bar{C}_{1}$.

We argue by contradiction. Indeed, suppose we have a closed geodesic $\gamma:[0, L] \rightarrow M^{n}$ of length $L<2 \bar{C}_{1}, X \circ \gamma$ must be contained in $\bar{B}\left(y_{0}, \bar{C}_{1}\right)$, then by $(2.5)$, we have

$$
\frac{d^{2}}{d s^{2}} f \circ \gamma(s)=\nabla^{2} f(\dot{\gamma}, \dot{\gamma}) \geq \frac{1}{2}, \quad s \in[0, L] .
$$


By the maximum principle, we have

$$
\sup _{s \in[0, L]} f \circ \gamma(s) \leq f \circ \gamma(0)
$$

this implies that $\gamma$ is just a point $\gamma(0)$. The contradiction proves the claim.

On the other hand, by the Gauss equation,

$$
R_{i j k l}=\bar{R}_{i j k l}+\left(h_{i k}^{\alpha} h_{j l}^{\beta}-h_{i l}^{\alpha} h_{k j}^{\beta}\right) \bar{g}_{\alpha \beta}(\cdot, 0),
$$

we see that

$$
|\mathrm{Rm}| \leq \bar{C}+2 C^{2}
$$

Finally, by Klingenberg lemma [3], the injectivity radius of $\left(M^{n}, g\right)$ at $x_{0}$ is given by

$$
\begin{aligned}
\operatorname{inj}\left(M^{n}, g, x_{0}\right)= & \min \left\{\text { the conjugate radius at } x_{0},\right. \\
& \left.\frac{1}{2} \text { the length of the shortest closed geodesic at } x_{0}\right\} \\
\geq & \min \left\{\frac{\pi}{\sqrt{\bar{C}+2 C^{2}}}, \bar{C}_{1}\right\} .
\end{aligned}
$$

The proof of the theorem is completed.

Let $N$ be a Riemannian manifold, the distance function $d\left(y_{1}, y_{2}\right)$ can be regarded as a function on $N \times N$. In the next theorem, we will estimate the Hessian of the distance function, which is viewed as the function of two variables. The crucial computation of the Hessian was carried out in [23].

Theorem 2.2. Let $N^{n}$ be a complete Riemannian manifold of dimension $n$ satisfying

$$
|\operatorname{Rm}| \leq K_{0}, \quad \operatorname{inj}\left(N^{n}\right) \geq i_{0}>0
$$

Let $d\left(y_{1}, y_{2}\right)$ be the distance function regarded as a function on $N \times N$, then there is a positive constant $C=C\left(K_{0}, i_{0}\right)$ such that when $d\left(y_{1}, y_{2}\right) \leq$ $\min \left\{\left(i_{0} / 2\right),\left(1 / 4 \sqrt{K_{0}}\right)\right\}$, we have

(i) $\left|\nabla^{2} d^{2}\right|\left(y_{1}, y_{2}\right) \leq C$,

(ii) $\left(\nabla^{2} d^{2}\right)(X, X) \geq 2\left|X_{1}-P_{\gamma}^{-1} X_{2}\right|^{2}-C|X|^{2} d^{2}$ for all $X \in T_{\left(y_{1}, y_{2}\right)} N^{n} \times N^{n}$, 
Uniqueness and pseudolocality theorems of the mean curvature flow 443

where $X=X_{1}+X_{2}, X_{1} \in T_{y_{1}} N^{n}, X_{2} \in T_{y_{2}} N^{n}, \nabla$ is the covariant derivative of $N \times N, \gamma$ is the unique geodesic connecting $y_{1}$ and $y_{2}$ in $N^{n}$, and $P_{\gamma}$ is the parallel translation of $N^{n}$ along $\gamma$.

Proof. Set $\psi\left(y_{1}, y_{2}\right)=d_{N^{n}}^{2}\left(y_{1}, y_{2}\right)$. Then $\psi$ is a smooth function of $\left(y_{1}, y_{2}\right)$ when $d\left(y_{1}, y_{2}\right) \leq \min \left\{\left(i_{0} / 2\right),\left(1 / 4 \sqrt{K_{0}}\right)\right\}$. Now we recall the computation of $\operatorname{Hess}(\psi)$ in [23]. For any $(u, v) \in D=\left\{(u, v):(u, v) \in N^{n} \times N^{n}, d_{N^{n}}(u, v) \leq\right.$ $\left.\min \left\{\left(i_{0} / 2\right),\left(1 / 4 \sqrt{K_{0}}\right)\right\}\right\} \backslash\left\{(u, u): u \in N^{n}\right\}$, let $\gamma_{u v}$ be the minimal geodesic from $u$ to $v$ and $e_{1} \in T_{u} N^{n}$ be the tangent vector to $\gamma_{u v}$ at $u$. Then $e_{1}(u, v)$ defines a smooth vector field on $D$. Let $\left\{e_{i}\right\}$ be an orthonormal basis for $T_{u} N^{n}$ which depends on $u$ smoothly. By parallel translation of $\left\{e_{i}\right\}$ along $\gamma$, we define $\left\{\bar{e}_{i}\right\}$ an orthonormal basis for $T_{v} N^{n}$. Thus $\left\{e_{1}, \ldots e_{n}, \bar{e}_{1}, \ldots \bar{e}_{n}\right\}$ is a local frame on $D$. Then for any $X=X_{1}+X_{2} \in T_{(u, v)} D$ with

$$
X_{1}=\sum_{i=1}^{n} \xi_{i} e_{i} \quad \text { and } \quad X_{2}=\sum_{i=1}^{n} \eta_{i} \bar{e}_{i}
$$

by the formula (16) in [23],

$$
\begin{aligned}
\frac{1}{2} \operatorname{Hess}(\psi)(X, X)= & \sum_{i=1}^{n}\left(\xi_{i}-\eta_{i}\right)^{2}+\int_{0}^{r} t\left\langle\nabla_{e_{1}} V, \nabla_{e_{1}} V\right\rangle \\
& +\int_{0}^{r} t\left\langle\nabla_{\bar{e}_{1}} V, \nabla_{\bar{e}_{1}} V\right\rangle-\int_{0}^{r} t\left\langle R\left(e_{1}, V\right) V, e_{1}\right\rangle \\
& -\int_{0}^{r} t\left\langle R\left(\bar{e}_{1}, V\right) V, \bar{e}_{1}\right\rangle
\end{aligned}
$$

where $V$ is a Jacobi field on geodesic $\sigma$ (connecting $(v, v)$ to $(u, v)$ ) and $\bar{\sigma}$ (connecting $(u, u)$ to $(u, v)$ of length $r=\sqrt{\psi}$ ) with $X$ as the boundary values, where $X$ is extended to be a local vector field by letting its coefficients with respect to $\left\{e_{1}, \ldots e_{n}, \bar{e}_{1}, \ldots \bar{e}_{n}\right\}$ be constant (see [23]). By the Jacobi equation, we have the estimates

$$
|V| \leq C\left(K_{0}, i_{0}\right)|X|, \quad r\left|\nabla_{e_{1}} V\right| \leq C\left(K_{0}, i_{0}\right)|X|, \quad r\left|\nabla_{\bar{e}_{1}} V\right| \leq C\left(K_{0}, i_{0}\right)|X|
$$

under the assumption $d\left(y_{1}, y_{2}\right) \leq \min \left\{\left(i_{0} / 2\right),\left(1 / 4 \sqrt{K_{0}}\right)\right\}$. Thus by $(2.10)$ we have

$$
|\operatorname{Hess}(\psi)| \leq C\left(K_{0}, i_{0}\right)
$$


this proves (i). Similarly, when $d\left(y_{1}, y_{2}\right) \leq \min \left\{\left(i_{0} / 2\right),\left(1 / 4 \sqrt{K_{0}}\right)\right\}$, by $(2.10)$, we have

$$
\begin{aligned}
\frac{1}{2} \operatorname{Hess}(\psi)(X, X) \geq & \sum_{i=1}^{n}\left(\xi_{i}-\eta_{i}\right)^{2}-\int_{0}^{r} t\left\langle R\left(e_{1}, V\right) V, e_{1}\right\rangle \\
& -\int_{0}^{r} t\left\langle R\left(\bar{e}_{1}, V\right) V, \bar{e}_{1}\right\rangle \\
\geq & \sum_{i=1}^{n}\left(\xi_{i}-\eta_{i}\right)^{2}-C\left(K_{0}, i_{0}\right)|X|^{2} r^{2} .
\end{aligned}
$$

This proves (ii). The Theorem is proved.

For future applications, in the next part of this section, we will calculate the equations of derivatives of general harmonic map flow. Since the MCF is a kind of harmonic map flow with varying base metrics evolved by MCF, the formulas computed here are very useful in deriving the higher derivativeestimates in Section 3 and 4. The formulas are of interest in their own rights. First we fix some notations.

Let $F$ be a map from a Riemannian manifold $\left(M, g_{i j}\right)$ to another Riemannian manifold $\left(N, \bar{g}_{\alpha \beta}\right)$, let $F^{-1} T N$ be the pull back of the tangent bundle of $N$, we equip the bundle $\left(T^{*} M\right)^{\otimes p} \otimes F^{-1} T N$ the connection and metric induced from the connections and metrics of $M$ and $N$. Let $u$ be a section of $\left(T^{*} M\right)^{\otimes(p-1)} \otimes F^{-1} T N$. In local coordinates $\left\{x^{i}\right\}$ and $\left\{y^{\alpha}\right\}$ of $M$ and $N$ with $y=F(x)$, we have $|u|^{2}=u_{i_{1} i_{2} \ldots i_{p-1}}^{\alpha} u_{j_{1} j_{2} \ldots j_{p-1}}^{\beta} g^{i_{1} j_{1}} \ldots g^{i_{p-1} j_{p-1}} \bar{g}_{\alpha \beta}$. The coefficients of the covariant derivative $\nabla u$ can be computed by the formula

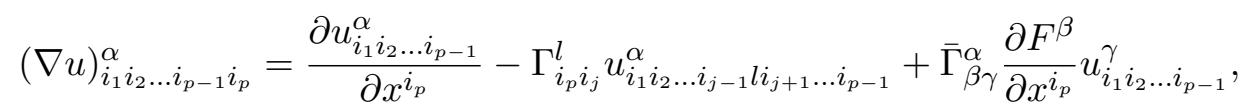

where $\Gamma$ and $\bar{\Gamma}$ are connection coefficients of $M$ and $N$, respectively. We can define the Laplacian of $u$ by $\Delta u=\operatorname{tr}_{g} \nabla^{2} u=g^{i j}\left(\nabla^{2} u\right)_{\ldots i j}$. Recall the Ricci identity

$$
\begin{aligned}
\left(\nabla^{2} u\right)_{\cdots i j}^{\alpha}-\left(\nabla^{2} u\right)_{\cdots j i}^{\alpha}= & -R_{i j i_{m} l} u_{\cdots i_{m-1} k i_{m+1} \ldots}^{\alpha} g^{k l} \\
& +\bar{R}_{\beta \gamma \delta \zeta} \frac{\partial F^{\beta}}{\partial x^{j}} \frac{\partial F^{\gamma}}{\partial x^{i}} \bar{g}^{\alpha \delta} u_{\ldots}^{\zeta}
\end{aligned}
$$


Uniqueness and pseudolocality theorems of the mean curvature flow 445

Note that the derivative $\nabla F\left(\nabla_{i} F^{\alpha}=\left(\partial F^{\alpha} / \partial x^{i}\right)\right)$ is a section of the bundle $T^{*} M \otimes F^{-1} T N$, the higher-derivative $\nabla^{p} F$ is a section of $\left(T^{*} M\right)^{\otimes p} \otimes$ $F^{-1} T N$.

If we have a family of metrics $g_{i j}(\cdot, t)$ on $M$ and a family of maps $F(\cdot, t)$ from $M$ to $N$, then for each time $t$, we can still define the bundle $\left(T^{*} M\right)^{\otimes p} \otimes$ $F^{-1} T N$ and define the covariant derivative $\nabla$. It is a useful observation that the natural time derivative $(\partial / \partial t)$ is not covariant with the metrics. We define a covariant time derivative $D_{t}$ as follows. For any section $u_{i_{1} \ldots i_{p}}^{\alpha}$ of $\left(T^{*} M\right)^{\otimes p} \otimes F^{-1} T N$, we define

$$
D_{t} u_{i_{1} \ldots i_{p}}^{\alpha}=\frac{\partial}{\partial t} u_{i_{1} \ldots i_{p}}^{\alpha}+\bar{\Gamma}_{\beta \gamma}^{\alpha} \frac{\partial F^{\beta}}{\partial t} u_{i_{1} \ldots i_{p}}^{\gamma} .
$$

It is a routine computation which shows that the operator $D_{t}$ is covariant.

Proposition 2.3. Let $M$ be a manifold with a family of metrics $g_{i j}(x, t)$, $(N, \bar{g})$ a Riemannian manifold. Let $F(\cdot, t)$ be a solution to the harmonic map flow with respect to the evolving metrics $g_{t}$ and $\bar{g}$

$$
\frac{\partial}{\partial t} F(x, t)=\triangle F(x, t), \text { for } x \in M^{n} \text { and } t \geq 0,
$$

where $\triangle F(x, t)$ is the harmonic map Laplacian of $F$ defined by metrics $g_{i j}(x, t)$ and $\bar{g}$. Then we have

$$
\begin{aligned}
\left(D_{t}-\triangle\right) \nabla^{k} F= & \sum_{l=0}^{k-1} \nabla^{l}\left[\left(R_{M} * g^{-2}+\bar{R}_{N} *(\nabla F)^{2} * g^{-1} * \bar{g}^{-1}\right)\right] * \nabla^{k-l} F \\
& +\sum_{l=1}^{k-1} g^{-1} * \nabla^{l} \frac{\partial g}{\partial t} * \nabla^{k-l} F
\end{aligned}
$$

where $\nabla^{l}(A * B)$ represents the linear combinations of $\nabla^{l} A * B, \nabla^{l-1} A *$ $\nabla B, \ldots, A * \nabla^{l} B$ with universal coefficients.

Proof. For $k=1$, by direct computation and Ricci identity, we have

$$
\begin{aligned}
\frac{\partial}{\partial t} \nabla_{i} F^{\alpha}+\bar{\Gamma}_{\beta \gamma}^{\alpha} F_{i}^{\beta}(\triangle F)^{\gamma}= & \nabla_{i} \triangle F^{\alpha} \\
= & \triangle \nabla_{i} F^{\alpha}-R_{i}^{l} \nabla_{l} F^{\alpha} \\
& +\bar{R}_{\beta \delta \gamma}^{\alpha} \nabla_{i} F^{\beta} \nabla_{k} F^{\delta} \nabla_{l} F^{\gamma} g^{k l}
\end{aligned}
$$


For $k \geq 2$, we prove by induction. Since

$$
\begin{aligned}
\frac{\partial}{\partial t}\left(\nabla^{k} F\right)_{i_{1} \ldots i_{k}}^{\alpha}= & \frac{\partial}{\partial x^{i_{k}}} \frac{\partial}{\partial t}\left(\nabla^{k-1} F\right)_{i_{1} \ldots i_{k-1}}^{\alpha}-\Gamma_{i_{k} i_{l}}^{p} \frac{\partial}{\partial t}\left(\nabla^{k-1} F\right)_{i_{1} \ldots p \ldots i_{k-1}}^{\alpha} \\
& +\bar{\Gamma}_{\beta \gamma}^{\alpha} F_{i_{k}}^{\beta} \frac{\partial}{\partial t}\left(\nabla^{k-1} F\right)_{i_{1} \ldots i_{k-1}}^{\gamma} \\
& +\left(g^{-1} * \nabla \frac{\partial g}{\partial t} * \nabla^{k-1} F\right)_{i_{1} \ldots i_{k}}^{\alpha} \\
& +\frac{\partial}{\partial y^{\delta}} \bar{\Gamma}_{\beta \gamma}^{\alpha}(\triangle F)^{\delta} F_{i_{k}}^{\beta}\left(\nabla^{k-1} F\right)_{i_{1} \ldots i_{k-1}}^{\gamma} \\
& +\bar{\Gamma}_{\beta \gamma}^{\alpha} \frac{\partial}{\partial t} F_{i_{k}}^{\beta}\left(\nabla^{k-1} F\right)_{i_{1} \ldots i_{k-1}}^{\gamma},
\end{aligned}
$$

we have

$$
\begin{aligned}
D_{t}\left(\nabla^{k} F\right)_{i_{1} \ldots i_{k}}^{\alpha}= & \frac{\partial}{\partial x^{i_{k}}} D_{t}\left(\nabla^{k-1} F\right)_{i_{1} \ldots i_{k-1}}^{\alpha}-\Gamma_{i_{k} i_{l}}^{p} D_{t}\left(\nabla^{k-1} F\right)_{i_{1} \ldots p \ldots i_{k-1}}^{\alpha} \\
& +\bar{\Gamma}_{\beta \gamma}^{\alpha} F_{i_{k}}^{\beta} D_{t}\left(\nabla^{k-1} F\right)_{i_{1} \ldots i_{k-1}}^{\gamma} \\
& +\left(g^{-1} * \nabla \frac{\partial g}{\partial t} * \nabla^{k-1} F\right)_{i_{1} \ldots i_{k}}^{\alpha} \\
& +\frac{\partial}{\partial y^{\delta}} \bar{\Gamma}_{\beta \gamma}^{\alpha}(\triangle F)^{\delta} F_{i_{k}}^{\beta}\left(\nabla^{k-1} F\right)_{i_{1} \ldots i_{k-1}}^{\gamma} \\
& +\bar{\Gamma}_{\beta \gamma}^{\alpha} \frac{\partial}{\partial t} F_{i_{k}}^{\beta}\left(\nabla^{k-1} F\right)_{i_{1} \ldots i_{k-1}}^{\gamma} \\
& -\frac{\partial}{\partial x^{i_{k}}}\left[\bar{\Gamma}_{\beta \gamma}^{\alpha} \frac{\partial F^{\beta}}{\partial t}\left(\nabla^{k-1} F\right)_{i_{1} \ldots i_{k-1}}^{\gamma}\right] \\
& +\Gamma_{i_{k} i_{l}}^{p} \bar{\Gamma}_{\beta \gamma}^{\alpha} \frac{\partial F^{\beta}}{\partial t}\left(\nabla^{k-1} F\right)_{i_{1} \ldots p \ldots i_{k-1}}^{\gamma} \\
& -\bar{\Gamma}_{\beta \gamma}^{\alpha} \bar{\Gamma}_{\delta \xi}^{\gamma} F_{i_{k}}^{\beta} \frac{\partial F^{\delta}}{\partial t}\left(\nabla^{k-1} F\right)_{i_{1} \ldots i_{k-1}}^{\xi}+\bar{\Gamma}_{\beta \gamma}^{\alpha} \frac{\partial F^{\beta}}{\partial t}\left(\nabla^{k} F\right)_{i_{1} \ldots i_{k}}^{\gamma}
\end{aligned}
$$

Since

$$
\begin{aligned}
\frac{\partial}{\partial x^{i_{k}}} & {\left[\bar{\Gamma}_{\beta \gamma}^{\alpha} \frac{\partial F^{\beta}}{\partial t}\left(\nabla^{k-1} F\right)_{i_{1} \ldots i_{k-1}}^{\gamma}\right]=\frac{\partial}{\partial y^{\beta}} \bar{\Gamma}_{\delta \gamma}^{\alpha} F_{i_{k}}^{\beta} \frac{\partial F^{\delta}}{\partial t}\left(\nabla^{k-1} F\right)_{i_{1} \ldots i_{k-1}}^{\gamma} } \\
& +\bar{\Gamma}_{\beta \gamma}^{\alpha} \frac{\partial}{\partial x^{i_{k}}} \frac{\partial F^{\beta}}{\partial t}\left(\nabla^{k-1} F\right)_{i_{1} \ldots i_{k-1}}^{\gamma}+\bar{\Gamma}_{\beta \gamma}^{\alpha} \frac{\partial F^{\beta}}{\partial t}\left(\nabla^{k} F\right)_{i_{1} \ldots i_{k}}^{\gamma} \\
& +\Gamma_{i_{k} i_{l}}^{p} \bar{\Gamma}_{\beta \gamma}^{\alpha} \frac{\partial F^{\beta}}{\partial t}\left(\nabla^{k-1} F\right)_{i_{1} \ldots p \ldots i_{k-1}}^{\gamma}-\bar{\Gamma}_{\delta \gamma}^{\alpha} \bar{\Gamma}_{\beta \xi}^{\gamma} F_{i_{k}}^{\beta} \frac{\partial F^{\delta}}{\partial t}\left(\nabla^{k-1} F\right)_{i_{1} \ldots i_{k-1}}^{\xi}
\end{aligned}
$$


Uniqueness and pseudolocality theorems of the mean curvature flow 447 we have

$$
\begin{aligned}
D_{t}\left(\nabla^{k} F\right)_{i_{1} \ldots i_{k}}^{\alpha}= & {\left[\nabla D_{t}\left(\nabla^{k-1} F\right)\right]_{i_{1} \ldots i_{k}}^{\alpha}+\left(g^{-1} * \nabla \frac{\partial g}{\partial t} * \nabla^{k-1} F\right)_{i_{1} \ldots i_{k}}^{\alpha} } \\
& +\bar{R}_{\delta \beta \gamma}^{\alpha}(\triangle F)^{\delta} F_{i_{k}}^{\beta}\left(\nabla^{k-1} F\right)_{i_{1} \ldots i_{k-1}}^{\gamma} .
\end{aligned}
$$

Combining with Ricci identity

$$
\nabla \triangle \nabla^{k-1} F=\triangle \nabla^{k} F+\nabla\left[\left(R_{M} * g^{-2}+\bar{R}_{N} *(\nabla F)^{2} * g^{-1} * \bar{g}^{-1}\right) * \nabla^{k-1} F\right]
$$

and induction on $k$, we have

$$
\begin{aligned}
\left(D_{t}-\triangle\right)\left(\nabla^{k} F\right)= & g^{-1} * \nabla \frac{\partial g}{\partial t} * \nabla^{k-1} F \\
& +\bar{R}_{N} * \nabla F * \nabla^{2} F * \nabla^{k-1} F * g^{-1} * \bar{g}^{-1} \\
& +\nabla\left[\left(D_{t}-\triangle\right) \nabla^{k-1} F\right] \\
& +\nabla\left[\left(R_{M} * g^{-2}+\bar{R}_{N} *(\nabla F)^{2} * g^{-1} * \bar{g}^{-1}\right) * \nabla^{k-1} F\right] \\
= & \nabla\left[\left(D_{t}-\triangle\right) \nabla^{k-1} F\right] \\
& +\nabla\left[\left(R_{M} * g^{-2}+\bar{R}_{N} *(\nabla F)^{2} * g^{-1} * \bar{g}^{-1}\right) * \nabla^{k-1} F\right] \\
& +g^{-1} * \nabla \frac{\partial g}{\partial t} * \nabla^{k-1} F \\
= & \sum_{l=0}^{k-1} \nabla^{l}\left[\left(R_{M} * g^{-2}+\bar{R}_{N} *(\nabla F)^{2} * g^{-1} * \bar{g}^{-1}\right)\right] * \nabla^{k-l} F \\
& +\sum_{l=1}^{k-1} g^{-1} * \nabla^{l} \frac{\partial g}{\partial t} * \nabla^{k-l} F .
\end{aligned}
$$

We finish the proof of the proposition.

Corollary 2.4. Let $F(\cdot, t)$ be assumed as in Proposition 2.3. Then we have

$$
\begin{aligned}
\left(\frac{\partial}{\partial t}-\triangle\right)\left|\nabla^{k} F\right|^{2} \leq & -2\left|\nabla^{k+1} F\right|^{2}+\left\langle\sum _ { l = 0 } ^ { k - 1 } \left\{\nabla ^ { l } \left[\left( R_{M} * g^{-2}\right.\right.\right.\right. \\
& \left.\left.+\bar{R}_{N} *(\nabla F)^{2} * g^{-1} * \bar{g}^{-1}\right)\right] \\
& \left.\left.+g^{-1} * \nabla^{l} \frac{\partial g}{\partial t}\right\} * \nabla^{k-l} F, \nabla^{k} F\right\rangle \\
& +g^{-(k+1)} \frac{\partial g}{\partial t} *\left(\nabla^{k} F\right)^{2} * \bar{g} .
\end{aligned}
$$


Proof. Since $\left|\nabla^{k} F\right|^{2}=\left(\nabla^{k} F\right)_{i_{1} \ldots i_{k}}^{\alpha}\left(\nabla^{k} F\right)_{j_{1} \ldots j_{k}}^{\beta} g^{i_{1} j_{1}} \ldots g^{i_{k} j_{k}} \bar{g}_{\alpha \beta}$, and

$$
\begin{aligned}
\frac{\partial}{\partial t}\left|\nabla^{k} F\right|^{2}= & 2 \frac{\partial}{\partial t}\left(\nabla^{k} F\right)_{i_{1} \ldots i_{k}}^{\alpha}\left(\nabla^{k} F\right)_{j_{1} \ldots j_{k}}^{\beta} g^{i_{1} j_{1}} \ldots g^{i_{k} j_{k}} \bar{g}_{\alpha \beta} \\
& +\frac{\partial \bar{g}_{\alpha \beta}}{\partial y^{\delta}} \frac{\partial F^{\delta}}{\partial t}\left(\nabla^{k} F\right)_{i_{1} \ldots i_{k}}^{\alpha}\left(\nabla^{k} F\right)_{j_{1} \ldots j_{k}}^{\beta} g^{i_{1} j_{1}} \ldots g^{i_{k} j_{k}} \\
& +g^{-(k+1)} * \frac{\partial g}{\partial t} *\left(\nabla^{k} F\right)^{2} * \bar{g} \\
= & 2 D_{t}\left(\nabla^{k} F\right)_{i_{1} \ldots i_{k}}^{\alpha}\left(\nabla^{k} F\right)_{j_{1} \ldots j_{k}}^{\beta} g^{i_{1} j_{1}} \ldots g^{i_{k} j_{k}} \bar{g}_{\alpha \beta} \\
& +g^{-(k+1)} * \frac{\partial g}{\partial t} *\left(\nabla^{k} F\right)^{2} * \bar{g}
\end{aligned}
$$

then (2.14) follows from Proposition 2.3.

\section{Higher-derivative estimates for the mean curvature flow}

Now we come back to MCF, suppose $X(\cdot, t)$ is a solution to MCF equation $(1.2), g(\cdot, t)$ is the family of the induced metrics on $M^{n}$ from $\left(\bar{M}^{\bar{n}}, \bar{g}\right)$ by $X(\cdot, t)$, then

$$
\frac{\partial}{\partial t} g_{i j}=-2 H^{\alpha} h_{i j}^{\beta} \bar{g}_{\alpha \beta}
$$

Note that $(\partial g / \partial t)=\left(\nabla^{2} X\right)^{2} * \bar{g} * g^{-1}$ and $R_{M}=\bar{R}_{\bar{M}} *(\nabla X)^{4}+\left(\nabla^{2} X\right)^{2} *$ $\bar{g}$. Combining with corollary 2.4 , we have

Proposition 3.1. Let $\left(\bar{M}^{\bar{n}}, \bar{g}\right)$ be a Riemannian manifold of dimension $\bar{n}$. Let $X_{0}: M^{n} \rightarrow \bar{M}^{\bar{n}}$ be an isometrically immersed manifold in $\bar{M}^{\bar{n}}$. Suppose $X(x, t)$ is a solution of MCF on $M^{n} \times[0, T]$ with $X_{0}$ as initial data. Then

$$
\begin{aligned}
\left(\frac{\partial}{\partial t}-\triangle\right)\left|\nabla^{k} X\right|^{2} \leq & -2\left|\nabla^{k+1} X\right|^{2}+\left\langle\sum _ { l = 0 } ^ { k - 1 } \nabla ^ { l } \left[\left(\nabla^{2} X\right)^{2} * \bar{g} * g^{-2}\right.\right. \\
& +\bar{R}_{\bar{M}} *(\nabla X)^{4} * g^{-2} \\
& \left.\left.* \bar{g} * \bar{g}^{-1}\right] * \nabla^{k-l} X, \nabla^{k} X\right\rangle \\
& +g^{-(k+2)} * \bar{g}^{2} *\left(\nabla^{2} X\right)^{2} *\left(\nabla^{k} X\right)^{2}
\end{aligned}
$$


Uniqueness and pseudolocality theorems of the mean curvature flow 449

Now we are ready to derive the higher-derivative estimates of the second fundamental form of MCF provided that we have bounded the second fundamental form. Before deriving the higher-derivative estimates, we need to construct a family of cut-off functions $\xi_{k}$, which are used also in the next section. For each integer $k>0$, let $\xi_{k}$ be a smooth nonincreasing function from $(-\infty,+\infty)$ to $[0,1]$ so that $\xi_{k}(s)=1$ for $s \in\left(-\infty,(1 / 2)+\left(1 / 2^{k+1}\right)\right]$, and $\xi_{k}(s)=0$ for $s \in\left[(1 / 2)+\left(1 / 2^{k}\right),+\infty\right)$; moreover for any $\epsilon>0$ there exists a universal $C_{k, \epsilon}>0$ such that

$$
\left|\xi_{k}^{\prime}(s)\right|+\left|\xi_{k}^{\prime \prime}(s)\right| \leq C_{k, \epsilon} \xi_{k}(s)^{1-\epsilon} .
$$

Theorem 3.2. (local estimates) Let $\left(\bar{M}^{\bar{n}}, \bar{g}\right)$ be a complete Riemannian manifold of dimension $\bar{n}$. Let $X_{0}: M^{n} \rightarrow \bar{M}^{\bar{n}}$ be an isometrically immersed complete manifold in $\bar{M}^{\bar{n}}$. Suppose $X(x, t)$ is a solution to the mean curvature flow (1.1) on $M^{n} \times[0, T]$ with $X_{0}$ as initial data and with bounded second fundamental forms $\left|h_{i j}^{\alpha}\right| \leq \bar{C}$ on $[0, T]$. Then for any fixed $x_{0} \in M^{n}$ and any geodesic ball $B_{0}\left(x_{0}, a\right)$ of radius $a>0$ of initial metric $g_{i j}$, for any $k \geq 3$, we have

$$
\left|\nabla^{k} X\right|(x, t) \leq \frac{C_{k}}{t^{(k-2 / 2)}}, \quad \text { for all }(x, t) \in B_{0}\left(x_{0}, \frac{a}{2}\right) \times[0, T],
$$

where the constant $C_{k}$ depends on $\bar{C}, T, \bar{n}$, a and the bounds of the curvature and its covariant derivatives up to order $k-1$ of the ambient manifold $\bar{M}$ on its geodesic ball $B_{\bar{M}}\left(X_{0}\left(x_{0}\right), a+1+\sqrt{n} \bar{C} T\right)$.

Proof. Since $|(\partial / \partial t) X|=|H| \leq \sqrt{n} \bar{C}$, it is not hard to see that under the evolution of MCF, at any time $t \in[0, T], X_{t}\left(B_{0}\left(x_{0}, a\right)\right)$ is contained in $B_{\bar{M}}\left(X_{0}\left(x_{0}\right), a+1+\sqrt{n} \bar{C} T\right)$. For any fixed $a>0, k>0$, we denote by $C_{k}$ various constants depending only on $a, \bar{C}, T, \bar{n}$ and the bounds of the curvature and its covariant derivatives up to order $k-1$ of the ambient manifold $\bar{M}$ on its ball $B_{\bar{M}}\left(X_{0}\left(x_{0}\right), a+1+\sqrt{n} \bar{C} T\right)$.

By Proposition 3.1, we have

$$
\begin{aligned}
\left(\frac{\partial}{\partial t}-\triangle\right)\left|\nabla^{2} X\right|^{2} & \leq-2\left|\nabla^{3} X\right|^{2}+C_{2}+C_{2}\left|\nabla^{3} X\right| \\
& \leq-\left|\nabla^{3} X\right|^{2}+C_{2}
\end{aligned}
$$


and

$$
\begin{aligned}
\left(\frac{\partial}{\partial t}-\triangle\right)\left|\nabla^{3} X\right|^{2} \leq & -2\left|\nabla^{4} X\right|^{2}+C_{3}\left(\left|\nabla^{3} X\right|^{3}+\left|\nabla^{3} X\right|^{2}+\left|\nabla^{3} X\right|\right. \\
& \left.+\left|\nabla^{4} X\right|\left|\nabla^{3} X\right|\right) \\
\leq & -\left|\nabla^{4} X\right|^{2}+C_{3}\left|\nabla^{3} X\right|^{3}+C_{3}
\end{aligned}
$$

Combining (3.5) and (3.6), for any constant $A>0$ we have

$$
\begin{aligned}
\left(\frac{\partial}{\partial t}\right. & -\triangle)\left(\left(A+\left|\nabla^{2} X\right|^{2}\right)\left|\nabla^{3} X\right|^{2}\right) \leq\left(-\left|\nabla^{3} X\right|^{2}+C_{3}\right)\left|\nabla^{3} X\right|^{2} \\
& +8\left|\nabla^{3} X\right|^{2}\left|\nabla^{4} X\right|\left|\nabla^{2} X\right|+\left[-\left|\nabla^{4} X\right|^{2}\right. \\
& \left.+C_{3}\left|\nabla^{3} X\right|^{3}+C_{3}\right]\left(A+\left|\nabla^{2} X\right|^{2}\right) .
\end{aligned}
$$

Since $\left|\nabla^{2} X\right|^{2}$ is bounded by assumption, by choosing $A$ suitable large, let $u=\left(A+\left|\nabla^{2} X\right|^{2}\right)\left|\nabla^{3} X\right|^{2}$ and $v=t u$, we have

$$
\left(\frac{\partial}{\partial t}-\triangle\right) u \leq-\frac{1}{C_{3}} u^{2}+C_{3}
$$

and

$$
\left(\frac{\partial}{\partial t}-\triangle\right) v \leq \frac{1}{t}\left(-\frac{1}{C_{3}} v^{2}+C_{3}\right)
$$

Now we need a cut-off function technique as in [5]. Let $\xi(x)=\xi_{3}\left(d_{0}\left(x, x_{0}\right) / a\right)$, where $\xi_{3}$ is the cut-off function satisfying (3.3) for $k=3$. Then the function $\xi(x)$ satisfies

$$
\begin{gathered}
\xi(x)=1, \quad \text { for } x \in B_{0}\left(x_{0},\left(\frac{1}{2}+\frac{1}{2^{4}}\right) a\right), \\
\xi(x)=0, \quad \text { for } x \in M \backslash B_{0}\left(x_{0}, a\right), \\
\left|\nabla_{0} \xi\right|^{2} \leq C_{3} \xi \\
\left(\nabla_{0}^{2} \xi\right)_{i j} \geq-C_{3} \xi^{1 / 2} g_{i j}(\cdot, 0),
\end{gathered}
$$

where we used the Hessian comparison theorem. Since by Gauss equation, the curvature of the initial metric is bounded from below by a constant, which depends on $\bar{C}$ and the curvature bound on the ball $B_{\bar{M}}\left(X_{0}\left(x_{0}\right)\right.$, 
Uniqueness and pseudolocality theorems of the mean curvature flow 451 $a+1+\sqrt{n} \bar{C} T)$ of the ambient manifold. The last formula holds in the sense of support functions. Define $\phi(x, t)=\xi(x) v(x, t)$. Then we have

$$
\left(\frac{\partial}{\partial t}-\triangle\right) \phi \leq \frac{1}{t}\left(-\frac{1}{C_{3}} \xi v^{2}-t v \triangle \xi-2 t \nabla \xi \cdot \nabla v+C_{3} \xi\right)
$$

Suppose $\phi(x, t)$ achieves its maximum value over $M^{n} \times[0, T]$ at some point $\left(x_{1}, t_{1}\right) \in B\left(x_{0}, a\right) \times(0, T]$, i.e.,

$$
\phi\left(x_{1}, t_{1}\right)=\max _{M \times[0, T]} \phi(x, t) .
$$

Suppose the point $x_{1}$ does not lie in the cut locus of $x_{0}$, then

$$
\frac{\partial \phi}{\partial t}\left(x_{1}, t_{1}\right) \geq 0, \quad \nabla v\left(x_{1}, t_{1}\right)=-\frac{\nabla \xi}{\xi} v, \quad \triangle \phi\left(x_{1}, t_{1}\right) \leq 0 .
$$

By (3.10) and (3.11), at $\left(x_{1}, t_{1}\right)$ we have

$$
0 \leq-\frac{1}{C_{3}} \xi v^{2}-t_{1} v \triangle \xi+2 t_{1} \frac{|\nabla \xi|^{2}}{\xi} v+C_{3} \xi
$$

Note that the second fundamental form is bounded in $M^{n} \times[0, T]$, the metrics $g_{i j}(\cdot, t)$ are equivalent. Since

$$
\frac{\partial}{\partial t} \Gamma_{i j}^{k}=\left(g^{-1} * \nabla \frac{\partial g}{\partial t}\right)_{i j}^{k}=g^{-2} * \bar{g} * \nabla^{2} X * \nabla^{3} X
$$

we have

$$
\begin{aligned}
\left|\Gamma_{i j}^{k}\left(x_{1}, t_{1}\right)-\Gamma_{0 i j}^{k}\left(x_{1}\right)\right| & \leq C(\bar{n}) \bar{C} \int_{0}^{t_{1}}\left|\nabla^{3} X\right| d t \\
& \leq C(\bar{n}) \bar{C} \int_{0}^{t_{1}}\left(\frac{\phi}{\xi t}\right)^{1 / 2}\left(x_{1}, t\right) d t \\
& \leq C_{3} \frac{\phi\left(x_{1}, t_{1}\right)^{1 / 2}}{\xi\left(x_{1}\right)^{1 / 2}}
\end{aligned}
$$


where we used the fact that $\phi$ achieves its maximum at $\left(x_{1}, t_{1}\right)$. Thus at $\left(x_{1}, t_{1}\right)$, we have

$$
\begin{aligned}
-\triangle \xi & =-g^{i j} \nabla_{i} \nabla_{j} \xi \\
& =-g^{i j}\left(\nabla_{0 i} \nabla_{0 j} \xi+\left(\Gamma_{0 i j}^{k}-\Gamma_{i j}^{k}\right) \nabla_{0 k} \xi\right) \\
& \leq C_{3}+C_{3} \frac{\phi\left(x_{1}, t_{1}\right)^{1 / 2}}{\xi\left(x_{1}\right)^{1 / 2}}|\nabla \xi| .
\end{aligned}
$$

Substituting into (3.12), multiplying by $\xi\left(x_{1}\right)$ and combining with (3.9), we have at $\left(x_{1}, t_{1}\right)$

$$
\begin{aligned}
0 & \leq-\frac{1}{C_{3}} \xi^{2} v^{2}+\left(C_{3}+C_{3} \phi\left(x_{1}, t_{1}\right)^{1 / 2} \frac{|\nabla \xi|}{\xi^{1 / 2}}\right) \xi v+2 \frac{|\nabla \xi|^{2}}{\xi} \xi v+C_{3} \xi^{2} \\
& \leq-\frac{1}{C_{3}} \phi^{2}+C_{3} \phi^{3 / 2}+C_{3} \phi+C_{3} .
\end{aligned}
$$

This implies

$$
\phi\left(x_{1}, t_{1}\right) \leq C_{3}
$$

hence we have

$$
\left|\nabla^{3} X\right| \leq \frac{C_{3}}{t^{1 / 2}}
$$

on $B_{0}\left(x_{0},\left((1 / 2)+\left(1 / 2^{4}\right)\right) a\right) \times[0, T]$. If $x_{1}$ lies on the cut locus of $x_{0}$, then by applying a standard support function technique as in [22], the same estimate is still valid.

For higher-derivatives, we prove by induction. Fix $x_{0} \in M^{n}, a>0$, suppose

$$
\left|\nabla^{k} X\right| \leq \frac{C_{k}}{t^{k-2 / 2}}, \quad k=3, \ldots, m-1
$$

on $B_{0}\left(x_{0},\left((1 / 2)+\left(1 / 2^{k+1}\right)\right) a\right) \times[0, T]$. Now we prove the estimate for $k=m$. 
Uniqueness and pseudolocality theorems of the mean curvature flow 453

By induction hypothesis and Proposition 3.1, we have

$$
\begin{aligned}
\left(\frac{\partial}{\partial t}-\triangle\right)\left|\nabla^{m} X\right|^{2} \leq & -2\left|\nabla^{m+1} X\right|^{2}+\left\langle\sum _ { l = 0 } ^ { m - 1 } \nabla ^ { l } \left[\left(\nabla^{2} X\right)^{2} * \bar{g} * g^{-2}\right.\right. \\
& \left.\left.+\bar{R}_{\bar{M}} *(\nabla X)^{4} * g^{-2} * \bar{g} * \bar{g}^{-1}\right] * \nabla^{m-l} X, \nabla^{m} X\right\rangle \\
& +g^{-(m+2)} * \bar{g}^{2} *\left(\nabla^{2} X\right)^{2} *\left(\nabla^{m} X\right)^{2} \\
\leq & -2\left|\nabla^{m+1} X\right|^{2}+C_{m} \sum_{l=0}^{m-1}\left\{\sum_{l_{1}+l_{2}=l}\left|\nabla^{2+l_{1}} X\right|\left|\nabla^{2+l_{2}} X\right|\right. \\
& \left.+\sum\left|\nabla^{l_{1}+1} X\right|\left|\nabla^{l_{2}+1} X\right|\left|\nabla^{l_{3}+1} X\right|\left|\nabla^{l_{4}+1} X\right|\right\} \\
& \times\left|\nabla^{m-l} X\right|\left|\nabla^{m} X\right| \\
\leq & -2\left|\nabla^{m+1} X\right|^{2}+C_{m}\left[\left|\nabla^{m+1} X\right|\left|\nabla^{m} X\right|\right. \\
& \left.+\left(\left|\nabla^{3} X\right|+1\right)\left|\nabla^{m} X\right|^{2}+t^{-(m-2 / 2)}\left|\nabla^{m} X\right|\right] \\
\leq & -\left|\nabla^{m+1} X\right|^{2}+\frac{C_{m}}{t^{1 / 2}}\left|\nabla^{m} X\right|^{2}+C_{m} t^{-(m-2 / 2)}\left|\nabla^{m} X\right|
\end{aligned}
$$

and

$$
\begin{aligned}
\left(\frac{\partial}{\partial t}-\triangle\right)\left|\nabla^{m-1} X\right|^{2} \leq & -\left|\nabla^{m} X\right|^{2}+\frac{C_{m-1}}{t^{1 / 2}}\left|\nabla^{m-1} X\right|^{2} \\
& +C_{m-1} t^{-(m-3 / 2)}\left|\nabla^{m-1} X\right| \\
\leq & -\left|\nabla^{m} X\right|^{2}+\frac{C_{m-1}}{t^{m-3+(1 / 2)}}
\end{aligned}
$$

on $B_{0}\left(x_{0},\left((1 / 2)+\left(1 / 2^{m}\right)\right) a\right) \times[0, T]$.

Define

$$
\psi(x, t)=\left(A+t^{m-3}\left|\nabla^{m-1} X\right|^{2}\right)\left|\nabla^{m} X\right|^{2} t^{m-2}
$$

for $A$ to be determined later. Combining (3.14) and (3.15), we have for suitable large $A$ as before

$$
\begin{aligned}
\left(\frac{\partial}{\partial t}-\triangle\right) \psi \leq & \frac{2 m-5}{t} \psi+t^{m-3}\left|\nabla^{m} X\right|^{2} t^{m-2}\left(-\left|\nabla^{m} X\right|^{2}+\frac{C_{m-1}}{t^{m-3+(1 / 2)}}\right) \\
& +t^{m-2}\left(A+t^{m-3}\left|\nabla^{m-1} X\right|^{2}\right)\left(-\left|\nabla^{m+1} X\right|^{2}\right. \\
& \left.+\frac{C_{m}}{t^{1 / 2}}\left|\nabla^{m} X\right|^{2}+C_{m} t^{-(m-2 / 2)}\left|\nabla^{m} X\right|\right) \\
& +8 t^{2 m-5}\left|\nabla^{m-1} X\right|\left|\nabla^{m} X\right|^{2}\left|\nabla^{m+1} X\right|
\end{aligned}
$$




$$
\begin{aligned}
\leq & \frac{2 m-5}{t} \psi-\frac{1}{2 t}\left[t^{m-2}\left|\nabla^{m} X\right|^{2}\right]^{2} \\
& +\frac{C_{m}}{t^{1 / 2}}\left[t^{m-2}\left|\nabla^{m} X\right|^{2}\right]+C_{m}\left[t^{m-2}\left|\nabla^{m} X\right|^{2}\right]^{1 / 2} \\
\leq & \frac{1}{t}\left[-\frac{1}{C_{m}} \psi^{2}+C_{m} \psi+C_{m} \psi^{1 / 2}\right] \\
\leq & \frac{1}{t}\left[-\frac{1}{C_{m}} \psi^{2}+C_{m}\right]
\end{aligned}
$$

on $B_{0}\left(x_{0},\left((1 / 2)+\left(1 / 2^{m}\right)\right) a\right) \times[0, T]$. To apply the cut-off function technique to (3.16) as before, we note that by the estimate for $k=3$, we know that

$$
\left|\Gamma-\Gamma_{0}\right| \leq C(\bar{n}) \bar{C} \int_{0}^{T}\left|\nabla^{3} X\right| d t \leq C_{3} \int_{0}^{T} \frac{1}{\sqrt{t}} d t \leq C_{3} .
$$

By calculating the equation of $\xi_{m}\left(d_{0}\left(x_{0}, \cdot\right) / a\right) \psi$ using (3.16), and repeating the same procedure of applying maximum principle as before, we can prove that

$$
\xi_{m}\left(\frac{d_{0}\left(x_{0}, \cdot\right)}{a}\right) \psi \leq C_{m} \text { on } B_{0}\left(x_{0}, a\right) \times[0, T],
$$

which implies

$$
\left|\nabla^{m} X\right|(x, t) \leq \frac{C_{m}}{t^{m-2 / 2}}, \quad \text { for all }(x, t) \in B_{0}\left(x_{0},\left(\frac{1}{2}+\frac{1}{2^{m+1}}\right) a\right) \times[0, T] .
$$

We complete the induction step and the theorem is proved.

Corollary 3.3. Let $\left(\bar{M}^{\bar{n}}, \bar{g}\right)$ be a complete Riemannian manifold satisfying

$$
\left|\bar{\nabla}^{k} \overline{R m}\right|(\cdot) \leq \bar{C}, \text { for } k \leq 2
$$

Let $X_{0}: M^{n} \rightarrow \bar{M}^{\bar{n}}$ be an isometrically immersed complete manifold in $\bar{M}^{\bar{n}}$. Suppose $X(\cdot, t)$ is a solution of $\mathrm{MCF}$ on $M^{n} \times[0, T]$ with $X_{0}$ as initial data and with bounded second fundamental forms $\left|h_{i j}^{\alpha}\right| \leq \bar{C}$ on $[0, T]$. Then there is a constant $C_{1}$ depending only on $\bar{C}, \bar{n}$ and $T$ such that

$$
|\nabla \mathrm{Rm}|(x, t) \leq \frac{C_{1}}{t^{1 / 2}}, \text { for all }(x, t) \in M^{n} \times[0, T] .
$$

Moreover, for any fixed $x_{0} \in M^{n}$ and any ball $B_{0}\left(x_{0}, a\right)$ of radius $a>0$ of initial metric $g_{i j}$, and for any $k \geq 2$, there is a constant $C_{k}$ depending only 
on $a, \bar{C}, \bar{n}, T$ and the bounds of the curvature and its derivatives up to order $k+1$ of the ambient manifold on its geodesic ball $B_{\bar{M}}\left(X_{0}\left(x_{0}\right), a+1+\right.$ $\sqrt{n} \bar{C} T)$, such that

$$
\left|\nabla^{k} R m\right|(x, t) \leq \frac{C_{k}}{t^{k / 2}}, \quad \text { for all }(x, t) \in B_{0}\left(x_{0}, \frac{a}{2}\right) \times[0, T] .
$$

Proof. This follows from Gauss equation and Theorem 3.2.

\section{Harmonic map flow coupled with mean curvature flow}

Let $X_{t}$ be the solution of MCF as in Theorem 1.1, $g_{i j}(x, t)$ the induced Riemannian metrics. Let $f: M^{n} \rightarrow N^{m}$ be a map from $M^{n}$ to a fixed Riemanian manifold $\left(N^{m}, \hat{g}_{\alpha \beta}\right)$. Then the harmonic map flow coupled with $\mathrm{MCF}$ is the following evolution equation of maps

$$
\begin{aligned}
\frac{\partial}{\partial t} f(x, t) & =\triangle f(x, t), \quad \text { for } x \in M^{n}, t>0, \\
f(x, 0) & =f(x), \quad \text { for } x \in M^{n},
\end{aligned}
$$

where the Harmonic map Laplacian $\triangle$ is defined by using the metric $g_{i j}(x, t)$ and $\hat{g}_{\alpha \beta}(y)$, i.e.,

$$
\triangle f^{\alpha}(x, t)=g^{i j}(x, t) \nabla_{i} \nabla_{j} f^{\alpha}(x, t),
$$

and

$$
\nabla_{i} \nabla_{j} f^{\alpha}=\frac{\partial^{2} f^{\alpha}}{\partial x^{i} \partial x^{j}}-\Gamma_{i j}^{k} \frac{\partial f^{\alpha}}{\partial x^{k}}+\hat{\Gamma}_{\beta \gamma}^{\alpha} \frac{\partial f^{\beta}}{\partial x^{i}} \frac{\partial f^{\gamma}}{\partial x^{j}} .
$$

Here we use $\left\{x^{i}\right\}$ and $\left\{y^{\alpha}\right\}$ to denote the local coordinates of $M^{n}$ and $N^{m}$ respectively, $\Gamma_{i j}^{k}$ and $\hat{\Gamma}_{\beta \gamma}^{\alpha}$ the corresponding Christoffel symbols of $g_{i j}$ and $\hat{g}_{\alpha \beta}$.

Now we fix a metric $\hat{g}=g(\cdot, T)$ on $M^{n}$, and let $\left(N^{m}, \hat{g}\right)=\left(M^{n}, \hat{g}\right)$. Note that the ambient manifold $(\bar{M}, \bar{g})$ in Theorem 1.1 satisfies the assumption of Corollary 3.3. By Corollary 3.3 and Theorem 2.1, we know that there are positive constants $\hat{C}_{1}, \hat{\delta}$ depending only on $\bar{C}, T, \bar{n}$ and $\bar{\delta}$ such that

$$
\begin{aligned}
& \left|\hat{R}_{N}\right|+\left|\hat{\nabla} \hat{R}_{N}\right| \leq \hat{C}_{1}, \\
& \operatorname{inj}(N, \hat{g}) \geq \hat{\delta}>0 .
\end{aligned}
$$

Moreover, by (3.18) of Corollary 3.3, for any fixed $y_{0} \in N$, for any $k \geq 2$, there is a constant $\hat{C}_{k}$ depending only on $\bar{C}, \bar{n}, T$ and the bounds of the 
curvature and its derivatives up to order $k+1$ of the ambient manifold on its ball $B_{\bar{M}}\left(X_{0}\left(y_{0}\right), 2 e^{\sqrt{n} \bar{C}^{2} T}+1+\sqrt{n} \bar{C} T\right)$, such that

$$
\left|\hat{\nabla}^{k} \hat{R}_{N}\right|(y) \leq \hat{C}_{k}, \text { for all } y \in \hat{B}\left(y_{0}, 1\right)
$$

In this section, we will establish the existence theorem for the above harmonic map flow coupled with MCF. More precisely, we will prove

Theorem 4.1. There exists $0<T_{0}<T$, depending only on $\bar{C}, T, \bar{n}, \bar{\delta}$, such that the harmonic map flow coupled with mean curvature flow

$$
\begin{aligned}
& \frac{\partial}{\partial t} F(x, t)=\Delta F(x, t), \quad x \in M^{n}, t>0, \\
& F(\cdot, 0)=\text { Identity, } x \in M^{n}
\end{aligned}
$$

has a solution on $M^{n} \times\left[0, T_{0}\right]$ such that the following estimates hold. There is a constant $C_{2}$ depending only on $\bar{C}, \bar{\delta}, \bar{n}$ and $T$ such that

$$
|\nabla F|+\left|\nabla^{2} F\right| \leq C_{2}
$$

For any $k \geq 3, B_{0}\left(x_{1}, 1\right) \subset M^{n}$, there is a constant $C_{k}$ depending only on $\bar{C}, \bar{\delta}, T, \bar{n}$ and $x_{1}$ such that

$$
\left|\nabla^{k} F\right| \leq C_{k} t^{-(k-2 / 2)} \text {, on } B_{0}\left(x_{1}, 1\right) \times\left[0, T_{0}\right]
$$

We will adapt the strategy of [5] by solving the corresponding initialboundary value problem on a sequence of exhausted bounded domains $D_{1} \subseteq$ $D_{2} \subseteq \cdots$ with smooth boundaries and $D_{j} \supseteq B_{0}\left(x_{0}, j+1\right)$,

$$
\begin{aligned}
\frac{\partial}{\partial t} F^{j}(x, t) & =\Delta F^{j}(x, t) \\
F^{j}(x, 0) & =x \text { for all } x \in D_{j}, \\
F^{j}(x, t) & =x \text { for all } x \in \partial D_{j},
\end{aligned}
$$

and taking a convergent subsequence of $F^{j}$ as $j \rightarrow \infty$, where $x_{0}$ is a fixed point in $M^{n}$.

First we need the zero order estimate for the Dirichlet problem (4.6). 
Uniqueness and pseudolocality theorems of the mean curvature flow 457

Lemma 4.2. There exist positive constants $T_{1}>0$ and $C>0$ such that for any $j$, if $F^{j}$ solves problem(4.6) on $\bar{D}_{j} \times\left[0, T_{1}^{\prime}\right]$ with $T_{1}^{\prime} \leq T_{1}$, then we have

$$
\hat{d}\left(x, F^{j}(x, t)\right) \leq C \sqrt{t}
$$

for any $(x, t) \in D_{j} \times\left[0, T_{1}^{\prime}\right]$, where $\hat{d}$ is the distance with respect to the metric $\hat{g}$.

Proof. For simplicity, we drop the superscript $j$. In the following argument, we denote by $C$ various positive constants depending only on the constants $\bar{C}, \bar{\delta}, T$, and $\bar{n}$ in Theorem 1.1. Note that $\hat{d}\left(y_{1}, y_{2}\right)$ is the distance function on the target $\left(M^{n}, \hat{g}\right)$, which can be regarded as a function on $M^{n} \times M^{n}$ with the product metric. Let $\varphi\left(y_{1}, y_{2}\right)=(1 / 2) \hat{d}^{2}\left(y_{1}, y_{2}\right)$ and $\rho(x, t)=\varphi(x, F(x, t))$. We compute

$$
\begin{aligned}
\left(\frac{\partial}{\partial t}-\Delta\right) \rho= & \hat{d}(x, F(x, t))\left(-\frac{\partial \hat{d}}{\partial y_{1}^{\alpha}} \Delta \mathrm{Id}^{\alpha}\right) \\
& -g^{i j}\left\{\frac{\partial^{2} \varphi}{\partial y_{1}^{\alpha} \partial y_{1}^{\beta}}-\left(\hat{\Gamma}_{\alpha \beta}^{\gamma} \circ \mathrm{Id}\right) \frac{\partial \varphi}{\partial y_{1}^{\gamma}}\right\} \frac{\partial \mathrm{Id}^{\alpha}}{\partial x^{i}} \frac{\partial \mathrm{Id}^{\beta}}{\partial x^{j}} \\
& -2 g^{i j} \frac{\partial^{2} \varphi}{\partial y_{1}^{\alpha} \partial y_{2}^{\beta}} \frac{\partial \mathrm{Id}^{\alpha}}{\partial x^{i}} \frac{\partial F^{\beta}}{\partial x^{j}} \\
& -g^{i j}\left\{\frac{\partial^{2} \varphi}{\partial y_{2}^{\alpha} \partial y_{2}^{\beta}}-\left(\hat{\Gamma}_{\alpha \beta}^{\gamma} \circ F\right) \frac{\partial \varphi}{\partial y_{2}^{\gamma}}\right\} \frac{\partial F^{\alpha}}{\partial x^{i}} \frac{\partial F^{\beta}}{\partial x^{j}} \\
= & -\hat{d} \frac{\partial \hat{d}}{\partial y_{1}^{\alpha}} \triangle \operatorname{Id}^{\alpha}-g^{i j} \operatorname{Hess}(\varphi)\left(V_{i}, V_{j}\right),
\end{aligned}
$$

where

$$
V_{i}=\frac{\partial \mathrm{Id}^{\alpha}}{\partial x^{i}} \frac{\partial}{\partial y_{1}^{\alpha}}+\frac{\partial F^{\alpha}}{\partial x^{i}} \frac{\partial}{\partial y_{2}^{\alpha}} .
$$

By Theorem 3.2, there is a constant $C$ depending only on $\bar{C}, T$ and $\bar{n}$ such that

$$
\left|\frac{\partial \Gamma}{\partial t}\right| \leq C\left|\nabla^{3} X\right| \leq \frac{C}{\sqrt{t}} .
$$

Since

$$
\Delta \mathrm{Id}=g^{-1} *(\hat{\Gamma} \circ \operatorname{Id}-\Gamma)=g^{-1} *(\Gamma(\cdot, T)-\Gamma(\cdot, t))
$$


then we have $|\Delta \mathrm{Id}| \leq C$ by (4.7), this implies

$$
\left(\frac{\partial}{\partial t}-\Delta\right) \rho \leq C \hat{d}-g^{i j} \operatorname{Hess}(\varphi)\left(V_{i}, V_{j}\right)
$$

By (4.1), the curvature of $\hat{g}$ is bounded by some constant $\hat{K}$, the injectivity radius of $\hat{g}$ has a uniform positive lower bound $\hat{\delta}$. We claim that if $\hat{d}(x, F(x, t)) \leq \min \{\hat{\delta} / 2,1 / 4 \sqrt{\hat{K}}\}$, then

$$
g^{i j} \operatorname{Hess}(\varphi)\left(V_{i}, V_{j}\right) \geq \frac{1}{2}|\nabla F|^{2}-C .
$$

Firstly, by Theorem 2.2(i), we have $|\operatorname{Hess}(\varphi)| \leq C$ under the assumption of the claim. On the other hand, the Hessian comparison theorem at those points not lying on the cut locus shows that

$$
\begin{aligned}
& \frac{\partial^{2} \varphi}{\partial y_{2}^{\alpha} \partial y_{2}^{\beta}}-\left(\hat{\Gamma}_{\alpha \beta}^{\gamma} \circ F\right) \frac{\partial \varphi}{\partial y_{2}^{\gamma}} \geq \frac{\pi}{4} \hat{g}_{\alpha \beta}, \\
& \frac{\partial^{2} \varphi}{\partial y_{1}^{\alpha} \partial y_{1}^{\beta}}-\left(\hat{\Gamma}_{\alpha \beta}^{\gamma} \circ I d\right) \frac{\partial \varphi}{\partial y_{1}^{\gamma}} \geq \frac{\pi}{4} \hat{g}_{\alpha \beta} .
\end{aligned}
$$

Combining the above inequalities, we have

$$
\begin{aligned}
g^{i j} \operatorname{Hess}(\varphi)\left(V_{i}, V_{j}\right) & \geq \frac{\pi}{4}|\nabla F|^{2}-C|\nabla F|-C \\
& \geq \frac{1}{2}|\nabla F|^{2}-C,
\end{aligned}
$$

which proves the claim. Hence when $\hat{d}(x, F(x, t)) \leq \min \{(\hat{\delta} / 2),(1 / 4 \sqrt{\hat{K}})\}$, we have

$$
\left(\frac{\partial}{\partial t}-\Delta\right) \rho \leq C \hat{d}-\frac{1}{2}|\nabla F|^{2}+C
$$

By maximum principle we have

$$
\hat{d}(x, F(x, t)) \leq C \sqrt{t} \text { whenever } \hat{d}(x, F(x, t)) \leq \min \left\{\frac{\hat{\delta}}{2}, \frac{1}{4 \sqrt{\hat{K}}}\right\} .
$$

Therefore there exists $T_{1} \leq\left(1 / C^{2}\right) \min ^{2}\{(\hat{\delta} / 2),(1 / 4 \sqrt{\hat{K}})\}$ such that

$$
\hat{d}(x, F(x, t)) \leq C \sqrt{t}, \text { for } t \leq T_{1}^{\prime}\left(\leq T_{1}\right),
$$

we have proved the lemma. 
Uniqueness and pseudolocality theorems of the mean curvature flow 459

After proving the above lemma, we can apply the standard parabolic equation theory to get a local existence for the initial-boundary value problem (4.6) as follows. This is similar to [5], we include the proof here for completeness.

Lemma 4.3. There exists a positive constant $T_{2}\left(\leq T_{1}\right)$ depending only on the dimension $n$, the constants $T_{1}$ and $C$ obtained in the previous lemma such that for each $j$, the initial-boundary value problem (4.6) has a smooth solution $F^{j}$ on $\bar{D}_{j} \times\left[0, T_{2}\right]$.

Proof. For an arbitrarily fixed point $\bar{x}$ in $M^{n}$, we consider the normal coordinates $\left\{x^{i}\right\}$ and $\left\{y^{\alpha}\right\}$ of the metric $g_{0 i j}$ and the metric $\hat{g}_{\alpha \beta}$, respectively, around $\bar{x}$. Locally the equation (4.6) is written as a system of equations

$$
\begin{aligned}
\frac{\partial y^{\alpha}}{\partial t}(x, t)= & g^{i j}(x, t)\left[\frac{\partial^{2} y^{\alpha}}{\partial x^{i} \partial x^{j}}-\Gamma_{i j}^{k}(x, t) \frac{\partial y^{\alpha}}{\partial x^{k}}\right. \\
& \left.+\hat{\Gamma}_{\beta \gamma}^{\alpha}\left(y^{1}(x, t), \ldots, y^{n}(x, t)\right) \frac{\partial y^{\beta}}{\partial x^{i}} \frac{\partial y^{\gamma}}{\partial x^{j}}\right] .
\end{aligned}
$$

Note that $\hat{\Gamma}_{\beta \gamma}^{\alpha}(\bar{x})=0$. Since by (4.1) the curvature of metric $\hat{g}$ and it's first covariant derivative are bounded on the whole target manifold, by applying Corollary 4.12 in [14], we know that there is some uniform constant $\hat{C}$ such that if $\hat{d}(y, \bar{x}) \leq(1 / \hat{C})$, then $\left|\hat{\Gamma}_{\beta \gamma}^{\alpha}(y)\right| \leq \hat{C} \hat{d}(y, \bar{x})$. (This fact is proved essentially in [14], although it is not explicitly stated.) By Lemma 4.2, $\hat{d}(x, F(x, t)) \leq C \sqrt{t}$, we conclude that the coefficients of the quadratic terms on the RHS of (4.9) can be as small as we like, provided $T_{2}>0$ is sufficiently small (independent of $\bar{x}$ and $j$ ).

Now for fixed $j$, we consider the corresponding parabolic system of the difference of the map $F^{j}$ and the identity map. Clearly the coefficients of the quadratic terms of the gradients are also very small. Thus, whenever (4.9) has a solution on a time interval $\left[0, T_{2}^{\prime}\right]$ with $T_{2}^{\prime} \leq T_{2}$, we can argue exactly as in the proof of Theorem 6.1 in Chapter VII of the book [19] to bound the norm of $\nabla F^{j}$ on the time interval $\left[0, T_{2}^{\prime}\right]$ by a positive constant depending only on $g_{0 i j}$, and $\hat{g}_{\alpha \beta}$ over the domain $D_{j+1}$, the $L^{\infty}$ bound of $F^{j}$ obtained in the previous lemma, and the boundary $\partial D_{j}$. Hence by the same argument as in the proof of Theorem 7.1 in Chapter VII of the book [19], we deduce that the initial-boundary value problem (4.9) has a smooth solution $F^{j}$ on $\bar{D}_{j} \times\left[0, T_{2}\right]$.

To get a convergent sequence of $F^{j}$, we need the following uniform estimates. 
Lemma 4.4. There exists a positive constant $T_{3}, 0<T_{3} \leq T_{2}$, independent of $j$, such that if $F^{j}$ solves

$$
\begin{aligned}
\frac{\partial}{\partial t} F^{j}(x, t) & =\Delta F^{j}(x, t) \text { on } D_{j} \times\left[0, T_{3}\right] \\
F^{j}(x, 0) & =x \text { on } D_{j} .
\end{aligned}
$$

Then for any $B_{0}\left(x_{1}, 1\right) \subset D_{j}$, there is a positive constant $C=C(\bar{C}, \bar{\delta}, \bar{n}, T)$ such that

$$
\left|\nabla F^{j}\right|+\left|\nabla^{2} F^{j}\right| \leq C
$$

on $B_{0}\left(x_{1},(1 / 2)\right) \times\left[0, T_{3}\right]$, and for any $k \geq 3$ there exist constants $C_{k}=$ $C\left(k, \bar{C}, \bar{\delta}, T, \bar{n}, x_{1}\right)$ satisfying

$$
\left|\nabla^{k} F^{j}\right| \leq C_{k} t^{-(k-2 / 2)}
$$

on $B_{0}\left(x_{1},(1 / 2)\right) \times\left[0, T_{3}\right]$.

Proof. We drop the superscript $j$. We denote by $C$ various constants depending only on $\bar{C}, \bar{\delta}, T, \bar{n}$. We first estimate $|\nabla F|$. By Corollary 2.4, we have

$$
\begin{aligned}
\left(\frac{\partial}{\partial t}-\triangle\right)|\nabla F|^{2} \leq & -2\left|\nabla^{2} F\right|^{2}+\left\langle\left(\left[R_{M} * g^{-2}+\hat{R}_{N} *(\nabla F)^{2} * g^{-1} * \hat{g}^{-1}\right]\right.\right. \\
& \left.\left.+g^{-1} * \frac{\partial g}{\partial t}\right) * \nabla F, \nabla F\right\rangle+g^{-2} \frac{\partial g}{\partial t} *(\nabla F)^{2} * \hat{g} .
\end{aligned}
$$

Note that $(\partial g / \partial t)=\left(\nabla^{2} X\right)^{2} * \bar{g} * g^{-1}, R_{M}=\bar{R}_{\bar{M}} *(\nabla X)^{4}+\left(\nabla^{2} X\right)^{2} * \bar{g}$, the second fundamental form $\nabla^{2} X$ and curvature $\bar{R}_{\bar{M}}$ are bounded by assumption, we know that $|(\partial g / \partial t)|$ and $\left|R_{M}\right|$ are bounded. The above formula gives

$$
\frac{\partial}{\partial t}|\nabla F|^{2} \leq \Delta|\nabla F|^{2}-2\left|\nabla^{2} F\right|^{2}+C|\nabla F|^{2}+C|\nabla F|^{4} .
$$

On the other hand, we know from (4.8) that

$$
\frac{\partial}{\partial t} \rho \leq \Delta \rho-\frac{1}{2}|\nabla F|^{2}+C
$$


Uniqueness and pseudolocality theorems of the mean curvature flow 461

where $\rho(x, t)=(1 / 2) \hat{d}^{2}(x, F(x, t))$. For any $a>0$ to be determined later, we compute

$$
\begin{aligned}
\frac{\partial}{\partial t}\left[(a+\rho)|\nabla F|^{2}\right] \leq & \Delta\left[(a+\rho)|\nabla F|^{2}\right]-2 \nabla \rho \cdot \nabla|\nabla F|^{2} \\
& -2(a+\rho)\left|\nabla^{2} F\right|^{2}+C(a+\rho)|\nabla F|^{2}+C(a+\rho)|\nabla F|^{4} \\
& -\frac{1}{2}|\nabla F|^{4}+C|\nabla F|^{2} .
\end{aligned}
$$

Since

$$
\begin{aligned}
-2 \nabla \rho \cdot \nabla|\nabla F|^{2} & \leq C \hat{d}\left(|\nabla F|+|\nabla F|^{2}\right)\left|\nabla^{2} F\right| \\
& \leq C\left(|\nabla F|^{2}+|\nabla F|^{4}\right) \hat{d}+C \hat{d}\left|\nabla^{2} F\right|^{2}
\end{aligned}
$$

and $\hat{d}(\cdot, F(\cdot, t)) \leq C \sqrt{t}$, by taking $a=(1 / 8 C)$ and $T_{3}$ suitable small, we have

$$
\frac{\partial}{\partial t}\left[(a+\rho)|\nabla F|^{2}\right] \leq \triangle\left[(a+\rho)|\nabla F|^{2}\right]-\frac{1}{8 C}\left|\nabla^{2} F\right|^{2}-\frac{1}{4}|\nabla F|^{4}+C
$$

for $t \leq T_{3}$. Let $u=(a+\rho)|\nabla F|^{2}$, then

$$
\frac{\partial u}{\partial t} \leq \Delta u-\frac{1}{C} u^{2}+C
$$

for $t \leq T_{3}$. Let $\xi(x)=\xi_{1}\left(d_{0}\left(x_{1}, x\right)\right)$ be a cut-off function, where $\xi_{1}$ is the nonincreasing smooth function in (3.3) supported in $[0,1)$ and equal to 1 in $\left[0, \frac{3}{4}\right]$. Note that at $t=0, u=a g^{i j}(\cdot, 0) g_{i j}(\cdot, T) \leq C$. Then by computing the equation of $\xi u$ and applying the maximum principle as before, we have

$$
\xi u(x, t) \leq C \text { on } M^{n} \times\left[0, T_{3}\right],
$$

this implies

$$
|\nabla F| \leq C \text { on } B_{0}\left(x_{1}, \frac{3}{4}\right) \times\left[0, T_{3}\right] .
$$


We now estimate $\left|\nabla^{2} F\right|$. By Corollary 2.4 again

$$
\begin{aligned}
\left(\frac{\partial}{\partial t}-\triangle\right)\left|\nabla^{2} F\right|^{2} \leq & -2\left|\nabla^{3} F\right|^{2}+\left\langle\sum _ { l = 0 } ^ { 1 } \left\{\nabla ^ { l } \left[\left( R_{M} * g^{-2}\right.\right.\right.\right. \\
& \left.\left.+\hat{R}_{N} *(\nabla F)^{2} * g^{-1} * \hat{g}^{-1}\right)\right] \\
& \left.\left.+g^{-1} * \nabla^{l} \frac{\partial g}{\partial t}\right\} * \nabla^{2-l} F, \nabla^{2} F\right\rangle \\
& +g^{-(3)} \frac{\partial g}{\partial t} *\left(\nabla^{2} F\right)^{2} * \hat{g}
\end{aligned}
$$

and by (3.4), (3.17) and (4.1), we know $\sqrt{t}\left|\nabla \frac{\partial g}{\partial t}\right|+\sqrt{t}\left|\nabla R_{M}\right|+\left|\hat{\nabla} \hat{R}_{N}\right|$ $\leq C$, and

$$
\frac{\partial}{\partial t}\left|\nabla^{2} F\right|^{2} \leq \Delta\left|\nabla^{2} F\right|^{2}-2\left|\nabla^{3} F\right|^{2}+C\left|\nabla^{2} F\right|^{2}+\frac{C}{\sqrt{t}}\left|\nabla^{2} F\right|
$$

on $B_{0}\left(x_{1}, \frac{3}{4}\right) \times\left[0, T_{3}\right]$. This implies

$$
\frac{\partial}{\partial t}\left|\nabla^{2} F\right| \leq \Delta\left|\nabla^{2} F\right|+C\left|\nabla^{2} F\right|+\frac{C}{\sqrt{t}} .
$$

By (4.10) we have

$$
\frac{\partial}{\partial t}|\nabla F|^{2} \leq \Delta|\nabla F|^{2}-2\left|\nabla^{2} F\right|^{2}+C
$$

Let

$$
u=\left|\nabla^{2} F\right|+|\nabla F|^{2}-2 C \sqrt{t}+2 C \sqrt{T}
$$

then

$$
\frac{\partial}{\partial t} u \leq \Delta u-u^{2}+C \text { on } B_{0}\left(x_{1}, \frac{3}{4}\right) \times\left[0, T_{3}\right] .
$$

Define the cut-off function $\xi(x)=\xi_{2}\left(d_{0}\left(x_{1}, x\right)\right)$. Note that at $t=0,\left|\nabla^{2} F\right|=$ $\left|\Gamma_{0}-\hat{\Gamma}\right| \leq C$, then $\left.u\right|_{t=0} \leq C$. Using the similar maximum principle argument as before, we get

$$
\xi u \leq C \text { on } B_{0}\left(x_{1}, \frac{1}{2}+\frac{1}{2^{2}}\right) \times\left[0, T_{3}\right],
$$

which implies

$$
\left|\nabla^{2} F\right| \leq C \text { on } B_{0}\left(x_{1}, \frac{1}{2}+\frac{1}{2^{3}}\right) \times\left[0, T_{3}\right]
$$


Uniqueness and pseudolocality theorems of the mean curvature flow 463

To derive the higher-derivative estimates we prove by induction on $k$. We denote by $C_{k}$ various constants, depending only on $\bar{C}, T, \bar{\delta}, \bar{n}$, and the bounds of the ambient manifold $\bar{M}$ curvature and its covariant derivatives up to order $k$ on its ball $B_{\bar{M}}\left(X_{0}\left(x_{1}\right), C\right)$ for suitable $C$.

Now suppose we have proved

$$
\left|\nabla^{l} F\right| \leq \frac{C_{l}}{t^{(l-2) / 2}}, \quad l=2, \ldots, k-1
$$

on $B_{0}\left(x_{1},\left((1 / 2)+\left(1 / 2^{k}\right)\right)\right) \times\left[0, T_{3}\right]$. By Corollaries 2.4 and 3.3, Theorem 3.2 and using (4.15), we get

$$
\frac{\partial}{\partial t}\left|\nabla^{k} F\right|^{2} \leq \Delta\left|\nabla^{k} F\right|^{2}-2\left|\nabla^{k+1} F\right|^{2}+C_{k}\left|\nabla^{k} F\right|^{2}+\frac{C_{k}}{t^{(k-1) / 2}}\left|\nabla^{k} F\right|,
$$

which implies

$$
\frac{\partial}{\partial t}\left|\nabla^{k} F\right| \leq \Delta\left|\nabla^{k} F\right|+C_{k}\left|\nabla^{k} F\right|+\frac{C_{k}}{t^{(k-1) / 2}} .
$$

We also have

$$
\frac{\partial}{\partial t}\left|\nabla^{k-1} F\right|^{2} \leq \Delta\left|\nabla^{k-1} F\right|^{2}-2\left|\nabla^{k} F\right|^{2}+\frac{C_{k-1}}{t^{k-(5 / 2)}} .
$$

Let

$$
u=t^{(k-2) / 2}\left|\nabla^{k} F\right|+t^{k-3}\left|\nabla^{k-1} F\right|^{2} .
$$

By combining (4.17) and (4.18), we obtain

$$
\frac{\partial}{\partial t} u \leq \Delta u-\frac{1}{t}\left(u^{2}+C_{k}\right)
$$

on $B_{0}\left(x_{1},\left((1 / 2)+\left(1 / 2^{k}\right)\right)\right) \times\left[0, T_{3}\right]$. Using the cut-off function $\xi(x)=\xi_{k}\left(d_{0}\right.$ $\left.\left(x_{1}, x\right)\right),(4.19)$ and applying maximum principle as before, we conclude with

$$
\left|\nabla^{k} F\right| \leq \frac{C_{k}}{t^{(k-2) / 2}} \text { on } B_{0}\left(x_{1},\left(\frac{1}{2}+\frac{1}{2^{k+1}}\right)\right) \times\left[0, T_{3}\right] .
$$

Therefore we complete the proof of Lemma 4.4.

Proof of Theorem 4.1. Now we combine the above three lemmas to prove Theorem 4.1. We have known that there is a $T_{3}>0$ such that for each $j$, 
the equation

$$
\begin{aligned}
\frac{\partial}{\partial t} F^{j}(x, t) & =\Delta F^{j}(x, t) \\
F^{j}(x, 0) & =x \text { for all } x \in D_{j}, \\
F^{j}(x, t) & =x \text { for all } x \in \partial D_{j}
\end{aligned}
$$

has a smooth solution $F^{j}$ on $\bar{D}_{j} \times\left[0, T_{3}\right]$. Since $D_{j} \supset B_{0}\left(x_{0}, j+1\right)$, by choosing any $x_{1} \in B_{0}\left(x_{0}, j\right)$ in Lemma 4.4 we have

$$
\left|\nabla F^{j}\right|+\left|\nabla^{2} F^{j}\right| \leq C
$$

on $B_{0}\left(x_{0}, j\right) \times\left[0, T_{3}\right]$, where $C$ depends only on $\bar{C}, \bar{n}, \bar{\delta}, T$. Moreover for any $x_{1} \in B_{0}\left(x_{0}, j\right), k \geq 3$, there is a $C_{k}$ depending on $\bar{C}, \bar{\delta}, T, \bar{n}$ and $x_{1}$ such that

$$
\left|\nabla^{k} F^{j}\right|\left(x_{1}, t\right) \leq C_{k} t^{-((k-2) / 2)} .
$$

Then we can take a convergent subsequence of $F^{j}($ as $j \rightarrow \infty)$ to get the desired $F$ with the desired estimates. So the proof of Theorem 4.1 is completed.

For later purpose, now we need to derive some preliminary estimate of $g_{i j}(x, t)$ with respect to $F^{*} \hat{g}$. Let $\hat{g}_{i j}=\left(F^{*} \hat{g}\right)_{i j}$.

Proposition 4.5. Under the assumption of Theorem 4.1, there exist $0<$ $T_{4} \leq T_{3}$ and $C>0$ depending only on $\bar{C}, \bar{n}, \bar{\delta}$ and $T$ such that for all $(x, t) \in$ $M^{n} \times\left[0, T_{4}\right]$, we have

$$
\frac{1}{C} \hat{g}_{i j}(x, t) \leq g_{i j}(x, t) \leq C \hat{g}_{i j}(x, t)
$$

Proof. Note that $|\nabla F|^{2}=\hat{g}_{i j} g^{i j} \leq C$, which implies $\hat{g}_{i j}(x, t) \leq C g_{i j}(x, t)$. For the reverse inequality, since the curvature of $g_{i j}(\cdot, t)$ is bounded, we compute the equation of $\hat{g}_{i j}(x, t)$ on the domain,

$$
\begin{aligned}
\frac{\partial}{\partial t} \hat{g}_{i j}= & \Delta \hat{g}_{i j}-R_{i k} F_{l}^{\alpha} F_{j}^{\beta} \hat{g}_{\alpha \beta} g^{k l}-R_{j k} F_{l}^{\alpha} F_{i}^{\beta} \hat{g}_{\alpha \beta} g^{k l} \\
& +2 \hat{R}_{\alpha \beta \gamma \delta} F_{i}^{\alpha} F_{k}^{\beta} F_{j}^{\gamma} F_{l}^{\delta} g^{k l}-2 \hat{g}_{\alpha \beta} F_{k i}^{\alpha} F_{l j}^{\beta} g^{k l} \\
\geq & \Delta \hat{g}_{i j}-R_{i k} \hat{g}_{j l} g^{k l}-R_{j k} \hat{g}_{i l} g^{k l}-C|\nabla F|^{2} \hat{g}_{i j}-2\left|\nabla^{2} F\right|^{2} g_{i j} \\
\geq & \Delta \hat{g}_{i j}-C g_{i j} .
\end{aligned}
$$


Uniqueness and pseudolocality theorems of the mean curvature flow 465

Note that for suitable large constant $C$, we have

$$
\frac{\partial}{\partial t} g_{i j} \leq C g_{i j}, \quad 0<t<T,
$$

and $\hat{g}_{i j} \geq \frac{1}{C} g_{i j}$ at time 0 . Thus for $t \leq 1 / C^{3}$, we have

$$
\left(\frac{\partial}{\partial t}-\triangle\right)\left(\hat{g}_{i j}+\left(C^{2} t-\frac{1}{C}\right) g_{i j}\right) \geq\left[-C+C^{2}+C\left(C^{2} t-\frac{1}{C}\right)\right] g_{i j} \geq 0 .
$$

Note that

$$
\left.\left(\hat{g}_{i j}+\left(C^{2} t-\frac{1}{C}\right) g_{i j}\right)\right|_{t=0} \geq 0 .
$$

Since $\left|\nabla^{2} X\right|+\sqrt{t}\left|\nabla^{3} X\right| \leq C$ and the curvature is bounded, then there is a smooth proper function $\varphi$ with $\varphi(x) \geq 1+d_{0}\left(x_{0}, x\right),|\nabla \varphi|+\left|\nabla^{2} \varphi\right| \leq C$. So Hamilton's maximum principle for tensors on complete manifolds is applicable, we get

$$
\hat{g}_{i j}+\left(C^{2} t-\frac{1}{C}\right) g_{i j} \geq 0 \text { for } t \leq \min \left\{T_{3}, C^{-3}\right\},
$$

which implies

$$
g_{i j} \leq 2 C \hat{g}_{i j}
$$

for $t \leq T_{4}=\min \left\{T_{3}, 1 / 2 C^{3}\right\}$.

The proof of the proposition is completed.

As a consequence, we know that the solution of the harmonic map flow coupled with the MCF is a family of diffeomorphisms.

Corollary 4.6. Let $F(x, t)$ be assumed as in the previous proposition. Then $F(\cdot, t)$ are diffeomorphisms from $M$ to $N$ for all $t \in\left[0, T_{4}\right]$.

Proof. Note that (4.20) implies that $F$ are local diffeomorphisms. For any $x_{1} \neq x_{2}$, we claim that $F\left(x_{1}, t\right) \neq F\left(x_{2}, t\right)$ for all $t \in\left[0, T_{4}\right]$. Suppose not; then there is the first time $t_{0}>0$ such that $F\left(x_{1}, t_{0}\right)=F\left(x_{2}, t_{0}\right)$. Choose small $\sigma>0$ so that there exist a neighborhood $\hat{O}$ of $F\left(x_{1}, t_{0}\right)$ and a neighborhood $O$ of $x_{1}$ such that $F^{-1}(\cdot, t)$ is a diffeomorphism from $\hat{O}$ to $O$ for each $t \in\left[t_{0}-\sigma, t_{0}\right]$, and let $\hat{\gamma}$ be a shortest geodesic (parametrized by arc 
length) on the target (with respect to the metric $\hat{g}$ ) with $\hat{\gamma}(0)=F\left(x_{1}, t\right)$, $\hat{\gamma}(l)=F\left(x_{2}, t\right)$ and $\hat{\gamma} \subset \hat{O}$. We compute

$$
\frac{\partial}{\partial t} \hat{d}\left(F\left(x_{1}, t\right), F\left(x_{2}, t\right)\right)=\left\langle V\left(F\left(x_{2}, t\right)\right), \hat{\gamma}^{\prime}(l)\right\rangle_{\hat{g}}-\left\langle V\left(F\left(x_{1}, t\right)\right), \hat{\gamma}^{\prime}(0)\right\rangle_{\hat{g}},
$$

where $V(F(x, t))=(\partial / \partial t) F(x, t)$. Now we pull back everything by $F^{-1}$ to $O$,

$$
\begin{aligned}
\frac{\partial}{\partial t} \hat{d}\left(F\left(x_{1}, t\right), F\left(x_{2}, t\right)\right) & =\left\langle P_{-\hat{\gamma}} V-V, \hat{\gamma}^{\prime}(0)\right\rangle_{F^{*} \hat{g}} \\
& \geq-\sup _{x \in F^{-1} \hat{\gamma}}|\hat{\nabla} V|(x, t) \hat{d}\left(F\left(x_{1}, t\right), F\left(x_{2}, t\right)\right),
\end{aligned}
$$

where $P_{\hat{\gamma}}$ is the parallel translation along $F^{-1} \hat{\gamma}$ using the connection defined by $F^{*} \hat{g}$. Since

$$
\hat{\nabla}_{k} V^{l}=\nabla_{k} V^{\alpha} \frac{\partial x^{l}}{\partial y^{\alpha}}
$$

where $\nabla_{k} V^{\alpha}$ is the covariant derivative of the section $V^{\alpha}$ of the bundle $F^{-1} T N$. Thus by (4.20) in proposition 4.5 , we have

$$
\left|\hat{\nabla}_{k} V^{l}\right|=\left[\nabla_{k} V^{\alpha} \nabla_{l} V^{\beta} \hat{g}_{\alpha \beta} \hat{g}^{k l}\right]^{1 / 2} \leq C\left|\nabla^{3} F\right| \leq \frac{C}{\sqrt{t}},
$$

where the constant $C$ depends on the $x_{1}$ and $x_{2}$ and is independent of $t$ by (4.5) of Theorem 4.1. Therefore, for $t \in\left[t_{0}-\sigma, t_{0}\right]$, we have

$$
\hat{d}\left(F\left(x_{1}, t\right), F\left(x_{2}, t\right)\right) \leq \mathrm{e}^{C\left(\sqrt{t_{0}}-\sqrt{t_{0}-\sigma}\right)} \hat{d}\left(F\left(x_{1}, t_{0}\right), F\left(x_{2}, t_{0}\right)\right)=0,
$$

which contradicts with the choice of $t_{0}$. The corollary is proved.

\section{Mean curvature De Turck flow}

From the previous section, we know that the harmonic map flow coupled with MCF with identity as initial data has a short-time solution $F(x, t)$ which maintains being a diffeomorphism with good estimates. Let $\bar{X}=$ $X \circ F^{-1}$ be a family of maps defined from $\left(N, \hat{g}_{\alpha \beta}\right)$ to $\bar{M}^{\bar{n}}$, then $\bar{X}$ satisfies the following mean curvature De Turck flow

$$
\frac{\partial}{\partial t} \bar{X}=g^{\alpha \beta} \hat{\nabla}_{\alpha} \hat{\nabla}_{\beta} \bar{X} \quad \text { for } y \in N
$$


Uniqueness and pseudolocality theorems of the mean curvature flow 467

where $g^{\alpha \beta}$ is the inverse matrix of $g_{\alpha \beta}(\cdot, t)=\left(\left(F^{-1}\right)^{*} g(\cdot, t)\right)_{\alpha \beta}, \hat{\nabla}$ is the covariant derivative with respect to $\hat{g}_{\alpha \beta}$. We denote the local coordinates of $\bar{M}$ by $\left\{\bar{z}^{\bar{\alpha}}\right\}$. It is not hard to see

$$
\begin{aligned}
g_{\alpha \beta}(y, t) & =g_{i j}(x, t) \frac{\partial x^{i}}{\partial y^{\alpha}} \frac{\partial x^{j}}{\partial y^{\beta}} \\
& =\bar{g}_{\bar{\alpha} \bar{\beta}} \frac{\partial X^{\bar{\alpha}}}{\partial x^{i}} \frac{\partial X^{\bar{\beta}}}{\partial x^{j}} \frac{\partial x^{i}}{\partial y^{\alpha}} \frac{\partial x^{j}}{\partial y^{\beta}}=\frac{\partial \bar{X} \bar{\gamma}}{\partial y^{\alpha}} \cdot \frac{\partial \bar{X}^{\bar{\delta}}}{\partial y^{\beta}} \bar{g}_{\bar{\gamma} \bar{\delta}}(\bar{X}(y, t)),
\end{aligned}
$$

this implies that the metric $g_{\alpha \beta}(y, t)$ is just the induced metric from the ambient space by the map $\bar{X}$. Since

$$
\hat{\Gamma}_{\alpha \beta}^{\gamma}(y)-\Gamma_{\alpha \beta}^{\gamma}(y, t)=\left(\nabla^{2} F\right)_{i j}^{\gamma} \frac{\partial x^{i}}{\partial y^{\alpha}} \frac{\partial x^{j}}{\partial y^{\beta}},
$$

we have

$$
\begin{aligned}
& \frac{1}{C} \hat{g}_{\alpha \beta}(y) \leq g_{\alpha \beta}(y, t) \leq C \hat{g}_{\alpha \beta}(y), \\
& \left|\hat{\Gamma}_{\alpha \beta}^{\gamma}(y)-\Gamma_{\alpha \beta}^{\gamma}(y, t)\right| \leq C,
\end{aligned}
$$

by Theorem 4.1 and Proposition 4.5.

Let $X_{1}$ and $X_{2}$ be two solutions of MCF with bounded second fundamental form and with the same initial value $X_{0}$ assumed as in the Theorem 1.1. Let $g_{i j}^{1}(x, t)$ and $g_{i j}^{2}(x, t)$ be the corresponding induced metrics. As in Section 4, we solve the harmonic map flows coupled with MCF with the same target $\left(M^{n}, \hat{g}_{\alpha \beta}\right)$, where $\hat{g}=g^{1}(T)$, respectively,

$$
\frac{\partial}{\partial t} F_{1}=\Delta_{g^{1}, \hat{g}} F_{1}
$$

$$
\left.F_{1}\right|_{t=0}=\text { Identity on } M^{n} \text {, }
$$

and

$$
\frac{\partial}{\partial t} F_{2}=\Delta_{g^{2}, \hat{g}} F_{2}
$$

$$
\left.F_{2}\right|_{t=0}=\text { Identity on } M^{n} \text {, }
$$


where $\Delta_{g^{k}, \hat{g}}$ is the harmonic map Laplacian defined by the metric $g_{i j}^{k}(x, t)$, and $\hat{g}_{\alpha \beta}$ for $k=1,2$, respectively. By Section 4, we obtain two solutions $F_{1}(x, t)$ and $F_{2}(x, t)$ such that Theorem 4.1 holds with $F=F_{1}$ and $F=F_{2}$. Corollary 4.6 says that $F_{1}(x, t)$ and $F_{2}(x, t)$ are diffeomorphisms for any $t \in\left[0, T_{4}\right]$. Let $g_{1_{\alpha \beta}}(y, t)=\left(\left(F_{1}^{-1}\right)^{*} g^{1}\right)_{\alpha \beta}(y, t)$ and $g_{2_{\alpha \beta}}(y, t)=\left(\left(F_{2}^{-1}\right)^{*} g^{2}\right)_{\alpha \beta}$ $(y, t)$. Then $\bar{X}_{1}=X_{1} \circ F_{1}^{-1}$ and $\bar{X}_{2}=X_{2} \circ F_{2}^{-1}$ are two solutions to the mean curvature De Turck flow (5.1) with the same initial value $X_{0}$,

$$
\begin{aligned}
& \frac{\partial}{\partial t} \bar{X}_{1}=g_{1}^{\alpha \beta} \hat{\nabla}_{\alpha} \hat{\nabla}_{\beta} \bar{X}_{1}, \text { on } M^{n} \times\left[0, T_{4}\right], \\
& \left.\bar{X}_{1}\right|_{t=0}=X_{0}, \quad \text { on } M^{n}, \\
& \frac{\partial}{\partial t} \bar{X}_{2}=g_{2}^{\alpha \beta} \hat{\nabla}_{\alpha} \hat{\nabla}_{\beta} \bar{X}_{2}, \quad \text { on } M^{n} \times\left[0, T_{4}\right], \\
& \left.\bar{X}_{2}\right|_{t=0}=X_{0}, \quad \text { on } M^{n},
\end{aligned}
$$

where $g_{1 \alpha \beta}$ and $g_{2 \alpha \beta}$ are the corresponding induced metrics from the target $\left(\bar{M}^{\bar{n}}, \bar{g}_{\bar{\alpha} \bar{\beta}}\right)$ by the maps $\bar{X}_{1}$ and $\bar{X}_{2}$ by $(5.2)$.

Proposition 5.1. Under the assumptions of Theorem 1.1, there is some $T_{5}>0$ depending only on $\bar{C}, \bar{\delta}, T$ and $\bar{n}$ such that

$$
\bar{X}_{1}(y, t)=\bar{X}_{2}(y, t) \text { on } M^{n} \times\left[0, T_{5}\right]
$$

for the two solutions of mean-De Turck flow constructed above.

Proof. Let $\psi\left(\overline{z_{1}}, \overline{z_{2}}\right)=d_{\bar{M}}^{2}\left(\bar{z}_{1}, \bar{z}_{2}\right)$ be the square of the distance function on $\bar{M}$ which is viewed as a function of $\left(\bar{z}_{1}, \bar{z}_{2}\right) \in \bar{M} \times \bar{M}$. Set $u(y, t)=$ $d_{\bar{M}}^{2}\left(\bar{X}_{1}(y, t), \bar{X}_{2}(y, t)\right)$. Let $\Delta_{k}=g_{k}^{\alpha \beta} \hat{\nabla}_{\alpha} \hat{\nabla}_{\beta}$ for $k=1,2$. By direct computation, we have

$$
\begin{aligned}
\frac{\partial}{\partial t} u(y, t)= & 2 d_{\bar{M}}\left(\bar{X}_{1}, \bar{X}_{2}\right) \frac{\partial d}{\partial \overline{z_{1}} \bar{\xi}} \triangle_{1} \bar{X}_{1}^{\bar{\xi}}+2 d_{\bar{M}}\left(\bar{X}_{1}, \bar{X}_{2}\right) \frac{\partial d}{\partial \overline{z_{2}} \bar{\zeta}} \triangle_{2} \bar{X}_{2}^{\bar{\zeta}}, \\
g_{1}^{\alpha \beta} \hat{\nabla}_{\alpha} \hat{\nabla}_{\beta} u(y, t)= & 2 d_{\bar{M}}\left(\bar{X}_{1}, \bar{X}_{2}\right)\left[\frac{\partial d}{\partial \overline{z_{1}}} \triangle_{1} \bar{X}_{1}^{\bar{\xi}}+\frac{\partial d}{\partial \overline{z_{2}} \bar{\zeta}} \triangle_{1} \bar{X}_{2}^{\bar{\zeta}}\right] \\
& +\operatorname{Hess}(\psi)\left(Z_{\alpha}, Z_{\beta}\right) g_{1}^{\alpha \beta}
\end{aligned}
$$

where $Z_{\alpha}=\left(\partial \bar{X}_{1}^{\bar{\xi}} / \partial y^{\alpha}\right)\left(\partial / \partial \bar{z}_{1}^{\bar{\xi}}\right)+\left(\partial \bar{X}_{2}^{\bar{\zeta}} / \partial y^{\alpha}\right)\left(\partial / \partial \bar{z}_{2}^{\bar{\zeta}}\right) \in T_{\left(\bar{X}_{1}, \bar{X}_{2}\right)} \bar{M} \times \bar{M}$, $\alpha=1,2, \ldots, n$ are vector fields on $\bar{M} \times \bar{M}$. Combining these two formulas, 
Uniqueness and pseudolocality theorems of the mean curvature flow 469

we have

$$
\begin{aligned}
{\left[\frac{\partial}{\partial t}-g_{1}^{\alpha \beta} \hat{\nabla}_{\alpha} \hat{\nabla}_{\beta}\right] u(y, t)=} & -2 d_{\bar{M}}\left(\bar{X}_{1}, \bar{X}_{2}\right) \frac{\partial d}{\partial \bar{z}_{2}^{\bar{\zeta}}}\left(\left(\triangle_{1}-\triangle_{2}\right) \bar{X}_{2}\right)^{\bar{\zeta}} \\
& -\operatorname{Hess}(\psi)\left(Z_{\alpha}, Z_{\beta}\right) g_{1}^{\alpha \beta} .
\end{aligned}
$$

Note that

$$
\begin{aligned}
\left(\triangle_{1}-\triangle_{2}\right) \bar{X}_{2} & =g_{1}^{\alpha \beta} \hat{\nabla}_{\alpha} \hat{\nabla}_{\beta} \bar{X}_{2}-g_{2}^{\alpha \beta} \hat{\nabla}_{\alpha} \hat{\nabla}_{\beta} \bar{X}_{2} \\
& =g_{1}^{\alpha \gamma} g_{2}^{\beta \delta}\left(g_{2 \delta \gamma}-g_{1 \delta \gamma}\right) \hat{\nabla}_{\alpha} \hat{\nabla}_{\beta} \bar{X}_{2}, \\
\hat{\nabla}_{\alpha} \hat{\nabla}_{\beta} \bar{X}_{2} & =\nabla_{2 \alpha} \nabla_{2 \beta} \bar{X}_{2}+\left(\hat{\Gamma}-\Gamma_{2}\right) * \nabla \bar{X}_{2},
\end{aligned}
$$

where $\Gamma_{2}$ and $\nabla_{2}$ are the Christoffel symbol and the covariant derivative of the metric $g_{2_{\alpha \beta}}(y, t)$.

For each $y \in M^{n}$ and $t \in[0, T]$, if $\bar{X}_{1}(y, t) \neq \bar{X}_{2}(y, t)$, denote the minimal geodesic on $\bar{M}$ from $\bar{X}_{1}(y, t)$ to $\bar{X}_{2}(y, t)$ by $\sigma$, and denote the parallel translation of $\bar{M}$ along $\sigma$ by $P_{\sigma}$, then we have

$$
\begin{aligned}
g_{1 \delta \gamma}(y, t)-g_{2 \delta \gamma}(y, t)= & \left\langle\overline{X_{1 *}}\left(\frac{\partial}{\partial y^{\delta}}\right), \overline{X_{1 *}}\left(\frac{\partial}{\partial y^{\gamma}}\right)\right\rangle_{\bar{g}} \\
& -\left\langle\overline{X_{2 *}}\left(\frac{\partial}{\partial y^{\delta}}\right), \overline{X_{2 *}}\left(\frac{\partial}{\partial y^{\gamma}}\right)\right\rangle_{\bar{g}} \\
= & \left\langle\overline{X_{1 *}}\left(\frac{\partial}{\partial y^{\delta}}\right), \overline{X_{1 *}}\left(\frac{\partial}{\partial y^{\gamma}}\right)\right\rangle_{\bar{g}} \\
& -\left\langle P_{\sigma}^{-1}\left(\overline{X_{2 *}}\left(\frac{\partial}{\partial y^{\delta}}\right)\right), P_{\sigma}^{-1}\left(\overline{X_{2 *}}\left(\frac{\partial}{\partial y^{\gamma}}\right)\right)\right\rangle_{\bar{g}} \\
= & \left\langle\overline{X_{1 *}}\left(\frac{\partial}{\partial y^{\delta}}\right)-P_{\sigma}^{-1}\left(\overline{X_{2 *}}\left(\frac{\partial}{\partial y^{\delta}}\right)\right), \overline{X_{1 *}}\left(\frac{\partial}{\partial y^{\gamma}}\right)\right\rangle_{\bar{g}} \\
& +\left\langle P_{\sigma}^{-1}\left(\overline{X_{2 *}}\left(\frac{\partial}{\partial y^{\delta}}\right)\right), \overline{X_{1 *}}\left(\frac{\partial}{\partial y^{\gamma}}\right)\right. \\
& \left.-P_{\sigma}^{-1}\left(\overline{X_{2 *}}\left(\frac{\partial}{\partial y^{\gamma}}\right)\right)\right\rangle_{\bar{g}} .
\end{aligned}
$$

If $\bar{X}_{1}(y, t)=\bar{X}_{2}(y, t), P_{\sigma}=$ Identity, the above formula still holds. 
In the following argument, we compute norms by using the metrics $g_{1}$ and $\bar{g}$. For example,

$$
\left|\hat{\Gamma}-\Gamma_{2}\right|^{2}=\left(\hat{\Gamma}-\Gamma_{2}\right)_{\alpha \beta}^{\gamma}\left(\hat{\Gamma}-\Gamma_{2}\right)_{\alpha^{\prime} \beta^{\prime}}^{\gamma^{\prime}} g_{1 \gamma \gamma^{\prime}} g_{1}^{\alpha \alpha^{\prime}} g_{1}^{\beta \beta^{\prime}}
$$

and

$$
\left|\nabla_{2}^{2} \bar{X}_{2}\right|^{2}=\bar{g}_{\bar{\xi} \bar{\zeta}} g_{1}^{\alpha \alpha^{\prime}} g_{1}^{\beta \beta^{\prime}} \nabla_{2 \alpha} \nabla_{2 \beta} \bar{X}_{2}^{\bar{\xi}} \nabla_{2 \alpha^{\prime}} \nabla_{2 \beta^{\prime}} \bar{X}_{2}^{\bar{\zeta}} .
$$

We denote by $C$ various constants depending only on the constants $\bar{C}, T, \bar{n}$ and $\bar{\delta}$ in the main Theorem 1.1. Then by (5.3), we have

$$
\begin{aligned}
& \left|\hat{\Gamma}-\Gamma_{2}\right| \leq C, \\
& \left|\hat{\nabla}^{2} \bar{X}_{2}\right| \leq C\left|\hat{\Gamma}-\Gamma_{2}\right|+C\left|\nabla_{2}^{2} \bar{X}_{2}\right| \leq C, \\
& \left|g_{2}\right|+\left|g_{2}^{-1}\right| \leq C,
\end{aligned}
$$

where $\left|\nabla_{2}^{2} \bar{X}_{2}\right|$ is just the norm of the second fundamental form of $X_{2}$ : $M^{n} \rightarrow \bar{M}^{\bar{n}}$ which is bounded by $\bar{C}$. Combining (5.9) (5.10) and (5.11), we have

$$
\begin{aligned}
\left|\left(\triangle_{1}-\triangle_{2}\right) \bar{X}_{2}\right|^{2} \leq & C g_{1}^{\delta \gamma}\left\langle\overline{X_{1 *}}\left(\frac{\partial}{\partial y^{\delta}}\right)-P_{\sigma}^{-1}\left(\overline{X_{2 *}}\left(\frac{\partial}{\partial y^{\delta}}\right)\right), \overline{X_{1 *}}\left(\frac{\partial}{\partial y^{\gamma}}\right)\right. \\
& \left.-P_{\sigma}^{-1}\left(\overline{X_{2 *}}\left(\frac{\partial}{\partial y^{\gamma}}\right)\right)\right\rangle_{\bar{g}} .
\end{aligned}
$$

By choosing an orthonormal frame at $y$ so that $g_{1 \alpha \beta}=\delta_{\alpha \beta}$, then we have

$$
\operatorname{Hess}(\psi)\left(Z_{\alpha}, Z_{\beta}\right) g_{1}^{\alpha \beta}=\sum_{\alpha=1}^{n} \operatorname{Hess}(\psi)\left(Z_{\alpha}, Z_{\alpha}\right) .
$$

Note that

$$
Z_{\alpha}=Z_{\alpha 1}+Z_{\alpha 2}, \text { for } \alpha=1,2, \ldots, n,
$$

where $Z_{\alpha 1}=\left(\partial \bar{X}_{1}^{\bar{\xi}} / \partial y^{\alpha}\right)\left(\partial / \partial \bar{z}_{1}^{\bar{\xi}}\right)=\overline{X_{1 *}}\left(\partial / \partial y^{\alpha}\right) \quad$ and $\quad Z_{\alpha 2}=\left(\partial \bar{X}_{2}^{\bar{\zeta}} / \partial y^{\alpha}\right)$ $\left(\partial / \partial \bar{z}_{2}^{\bar{\zeta}}\right)=\overline{X_{2 *}}\left(\partial / \partial y^{\alpha}\right)$.

Recall that by Theorem 2.2(ii), there is a constant $C$ such that if $d_{\bar{M}}\left(\bar{z}_{1}\right.$, $\left.\bar{z}_{2}\right) \leq \min \{(1 / 4 \sqrt{\bar{C}}),(\bar{\delta} / 2)\}$, we have

$$
\left(\nabla^{2} d^{2}\right)(Z, Z) \geq 2\left|Z_{1}-P_{\sigma}^{-1} Z_{2}\right|^{2}-C|Z|^{2} d^{2} \text { for all } Z \in T_{\left(\bar{z}_{1}, \bar{z}_{2}\right)} \bar{M}^{\bar{n}} \times \bar{M}^{\bar{n}},
$$


Uniqueness and pseudolocality theorems of the mean curvature flow 471

where $Z=Z_{1}+Z_{2}, \quad Z_{1} \in T_{\bar{z}_{1}} \bar{M}^{\bar{n}}, \quad Z_{2} \in T_{\bar{z}_{2}} \bar{M}^{\bar{n}}$. Hence if $d_{\bar{M}}\left(\bar{X}_{1}, \bar{X}_{2}\right) \leq$ $\min \{(1 / 4 \sqrt{\bar{C}}),(\bar{\delta} / 2)\}$, then

$$
\begin{aligned}
\sum_{\alpha=1}^{n} \operatorname{Hess}(\psi)\left(Z_{\alpha}, Z_{\alpha}\right) \geq & \sum_{\alpha=1}^{n} 2\left|\overline{X_{1 *}}\left(\frac{\partial}{\partial y^{\alpha}}\right)-P_{\sigma}^{-1} \bar{X}_{2 *}\left(\frac{\partial}{\partial y^{\alpha}}\right)\right|^{2} \\
& -C d_{\bar{M}}\left(\bar{X}_{1}, \bar{X}_{2}\right)^{2}
\end{aligned}
$$

since $\left|Z_{\alpha}\right| \leq C$.

Combining (5.8), (5.12) and (5.13), if $u^{1 / 2} \leq \min \{(1 / 4 \sqrt{\bar{C}}),(\bar{\delta} / 2)\}$, then we have

$$
\begin{aligned}
\left(\frac{\partial}{\partial t}-g_{1}^{\alpha \beta} \hat{\nabla}_{\alpha} \hat{\nabla}_{\beta}\right) u(y, t) \leq & C d_{\bar{M}}\left(\bar{X}_{1}, \bar{X}_{2}\right) \sum_{\alpha=1}^{n} 2 \mid \overline{X_{1 *}}\left(\frac{\partial}{\partial y^{\alpha}}\right) \\
& -P_{\sigma}^{-1} \bar{X}_{2 *}\left(\frac{\partial}{\partial y^{\alpha}}\right) \mid \\
& -2 \sum_{\alpha=1}^{n}\left|\overline{X_{1 *}}\left(\frac{\partial}{\partial y^{\alpha}}\right)-P_{\sigma}^{-1} \overline{X_{2 *}}\left(\frac{\partial}{\partial y^{\alpha}}\right)\right|^{2} \\
& +C d_{\bar{M}}^{2}\left(\bar{X}_{1}, \bar{X}_{2}\right) \\
\leq & C u .
\end{aligned}
$$

Now we show that $u^{1 / 2} \leq \min \{(1 / 4 \sqrt{\bar{C}}),(\bar{\delta} / 2)\}$ on some time interval $\left[0, T_{5}\right]$.

For any $(y, t) \in \hat{M} \times\left[0, T_{4}\right]$, we have

$$
\begin{aligned}
u^{1 / 2}(y, t) \leq & d_{\bar{M}}\left(X_{1} \circ F_{1}^{-1}(y, t), X_{1} \circ F_{1}^{-1}(y, 0)\right) \\
& +d_{\bar{M}}\left(X_{1} \circ F_{1}^{-1}(y, 0), X_{2} \circ F_{2}^{-1}(y, 0)\right) \\
& +d_{\bar{M}}\left(X_{2} \circ F_{2}^{-1}(y, t), X_{2} \circ F_{2}^{-1}(y, 0)\right) \\
\triangleq & I_{1}+I_{2}+I_{3} .
\end{aligned}
$$

By the mean curvature flow equation (1.1), we know

$$
I_{2} \leq d_{\bar{M}}\left(X_{1}(y, t), X_{1}(y, 0)\right)+d_{\bar{M}}\left(X_{2}(y, t), X_{2}(y, 0)\right) \leq 2 \sqrt{n} \bar{C} t .
$$

By (4.4) and (4.23), for any $x_{1}, x_{2} \in M^{n}$, we get

$$
\frac{\partial}{\partial t} \hat{d}\left(F_{1}\left(x_{1}, t\right), F_{1}\left(x_{2}, t\right)\right) \geq-C
$$

this implies

$$
\hat{d}\left(x_{1}, x_{2}\right) \leq \hat{d}\left(F_{1}\left(x_{1}, t\right), F_{1}\left(x_{2}, t\right)\right)+C t .
$$


By (5.16) and Lemma 4.2, it follows

$$
\begin{aligned}
I_{1} & =d_{\bar{M}}\left(X_{1} \circ F_{1}^{-1}(y, t), X_{1} \circ F_{1}^{-1}(y, 0)\right) \\
& \leq d_{\left(M, g^{1}(\cdot, t)\right)}\left(F_{1}^{-1}(y, t), y\right) \\
& \leq C \hat{d}\left(F_{1}^{-1}(y, t), y\right) \\
& \leq C t+C \hat{d}\left(y, F_{1}(y, t)\right) \\
& \leq C \sqrt{t}
\end{aligned}
$$

The estimate of $I_{3}$ is similar. Therefore, we have

$$
u^{1 / 2}(y, t) \leq C \sqrt{t}
$$

for some constant $C$ depending only on $\bar{C}, \bar{\delta}, T$ and $\bar{n}$.

Although $g_{1}^{\alpha \beta} \hat{\nabla}_{\alpha} \hat{\nabla}_{\beta}$ is not the standard Laplacian, the maximum principle is still applicable. For completeness, we include the proof in the following.

Since the curvature of $(M, \hat{g})$ is bounded, it is well-known that there is a function $\varphi$ such that

$$
\begin{gathered}
\frac{1}{C}\left(1+d_{\hat{g}}\left(y_{0}, y\right)\right) \leq \varphi(y) \leq C\left(1+d_{\hat{g}}\left(y_{0}, y\right)\right) \\
|\hat{\nabla} \varphi|+\left|\hat{\nabla}^{2} \varphi\right| \leq C
\end{gathered}
$$

Note $g_{1}$ is equivalent to $\hat{g}$. For any small $\varepsilon>0$ and large $A>0$, we have

$$
\left(\frac{\partial}{\partial t}-g_{1}^{\alpha \beta} \hat{\nabla}_{\alpha} \hat{\nabla}_{\beta}\right)\left(\mathrm{e}^{-C t} u(y, t)-\varepsilon \mathrm{e}^{A t} \varphi\right) \leq-\frac{\varepsilon A}{2} \mathrm{e}^{A t} \varphi<0 .
$$

Then the classical maximum principle implies that for any fixed $t_{0}$ the maximal value of $\left(\mathrm{e}^{-C t} u(y, t)-\varepsilon \mathrm{e}^{A t} \varphi\right)$ on $M \times\left[0, t_{0}\right]$ cannot be achieved for any point $(y, t)$ with $0<t \leq t_{0}$. Hence $\mathrm{e}^{-C t} u(y, t)-\varepsilon \mathrm{e}^{A t} \varphi \leq 0$ for any $t \in\left[0, T_{5}\right]$ for some $T_{5}>0$. Let $\varepsilon \rightarrow 0$, we conclude that $u \equiv 0$ on $\left[0, T_{5}\right]$. This implies $\bar{X}_{1}=\bar{X}_{2}$, on $M \times\left[0, T_{5}\right]$. We complete the proof of Proposition 5.1.

\section{Proof of the uniqueness Theorem 1.1}

Now we are ready to prove Theorem 1.1. Let $X_{1}(x, t)$ and $X_{2}(x, t)$ be two solutions of MCF with bounded second fundamental form and with the same initial data. We solve the corresponding harmonic map flow (5.4) and (5.5) (with the same target $(M, \hat{g}), \hat{g}=g_{1}(T)$ ), respectively, to obtain 
two solutions $F_{1}(x, t)$ and $F_{2}(x, t)$ on some common time interval. Then $\bar{X}_{1}=X_{1} \circ F_{1}^{-1}$ and $\bar{X}_{2}=X_{2} \circ F_{2}^{-1}$ are two solutions to the mean curvature De Turck flow with the same initial value. By Proposition 5.1 we know $\bar{X}_{1} \equiv \bar{X}_{2}$ on $\left[0, T_{5}\right]$. So in order to prove $X_{1}(x, t) \equiv X_{2}(x, t)$, we only need to show $F_{1} \equiv F_{2}$.

We know

$$
\begin{aligned}
& \Delta_{1} F_{1}^{\alpha}=g_{1}^{\beta \gamma}\left(\hat{\Gamma}_{\beta \gamma}^{\alpha}-\Gamma_{1 \beta \gamma}^{\alpha}\right) \circ F_{1}, \\
& \Delta_{2} F_{2}^{\alpha}=g_{2}^{\beta \gamma}\left(\hat{\Gamma}_{\beta \gamma}^{\alpha}-\Gamma_{2 \beta \gamma}^{\alpha}\right) \circ F_{2} .
\end{aligned}
$$

Since $\bar{X}_{1} \equiv \bar{X}_{2}$, we know $g_{1 \alpha \beta}(y, t)=g_{2 \alpha \beta}(y, t)$ on $\left[0, T_{5}\right]$, and the vector fields $V_{1} \equiv V_{2}$ on the target, where

$$
\begin{aligned}
& V_{1}^{\alpha}=g_{1}^{\beta \gamma}\left(\hat{\Gamma}_{\beta \gamma}^{\alpha}-\Gamma_{1 \beta \gamma}^{\alpha}\right), \\
& V_{2}^{\alpha}=g_{2}^{\beta \gamma}\left(\hat{\Gamma}_{\beta \gamma}^{\alpha}-\Gamma_{2 \beta \gamma}^{\alpha}\right) .
\end{aligned}
$$

Therefore, the two families of maps $F_{1}$ and $F_{2}$ satisfy the same ODE with the same initial value:

$$
\begin{aligned}
& \frac{\partial}{\partial t} F_{1}=V \circ F_{1} \\
& F_{1}(\cdot, 0)=\text { Identity }
\end{aligned}
$$

and

$$
\begin{aligned}
& \frac{\partial}{\partial t} F_{2}=V \circ F_{2} \\
& F_{2}(\cdot, 0)=\text { Identity. }
\end{aligned}
$$

So for any $x \in M^{n}$, letting $\gamma$ be a shortest geodesic (parametrized by arc length) on the target with $\gamma(0)=F_{1}(x, t)$ and $\gamma(l)=F_{2}(x, t)$, we have

$$
\begin{aligned}
\frac{\partial}{\partial t} \hat{d}\left(F_{1}(x, t), F_{2}(x, t)\right) & =\left\langle V, \gamma^{\prime}(l)\right\rangle-\left\langle V, \gamma^{\prime}(0)\right\rangle \\
& =\left\langle P_{\gamma}^{-1} V-V, \gamma^{\prime}(0)\right\rangle \\
& \leq \sup _{y \in \gamma}|\hat{\nabla} V|(y, t) \hat{d}\left(F_{1}(x, t), F_{2}(x, t)\right),
\end{aligned}
$$

where $P_{\gamma}^{-1} V$ is the parallel transport of $V\left(F_{2}(x, t), t\right)$ along the geodesic $\gamma$ back to the tangent space of the point $F_{1}(x, t)$. We have seen in the proof of Corollary 4.6 that $\sup _{y \in \gamma}|\hat{\nabla} V|(y, t) \leq \frac{C}{\sqrt{t}}$ for some $C$ depending on $x$ but 
independent of $t$. Since $\hat{d}\left(F_{1}(x, 0), F_{2}(x, 0)\right) \equiv 0$, we conclude that

$$
F_{1}(x, t) \equiv F_{2}(x, t)
$$

So we have proved $X_{1}(x, t)=X_{2}(x, t)$, for all $x \in M$ and $t \in\left[0, T_{5}\right]$. Clearly, we can extend the interval $\left[0, T_{5}\right]$ to the whole $[0, T]$ by applying the same argument on $\left[T_{5}, T\right]$.

The proof of Theorem 1.1 is completed.

Corollary 1.2 is a direct consequence of Theorem 1.1. Indeed, let $\bar{\sigma}$ and $\sigma$ be two isometries of $\left(\bar{M}^{\bar{n}}, \bar{g}\right)$ and $\left(M^{n}, g\right)$, respectively, such that $\left(\bar{\sigma} \circ X_{0}\right)(x)=\left(X_{0} \circ \sigma\right)(x)$ for any $x \in M^{n}$. Since $\bar{\sigma} \circ X_{t}$ and $X_{t} \circ \sigma$ are two solutions to the MCF (1.1) with bounded second fundamental form on $M^{n} \times$ $[0, T]$ and with the same initial value, then by Theorem 1.1 , we have

$$
\left(\bar{\sigma} \circ X_{t}\right)(x)=\left(X_{t} \circ \sigma\right)(x)
$$

for any $x \in M^{n}$ and $t \in[0, T]$. The proof of the Corollary 1.2 is completed.

\section{Pseudolocality theorem}

We begin with a few terminologies for the sake of convenience. An $n$-dimensional submanifold $M \subset \bar{M}$ is said to be a local $\delta$-Lipschitz graph of radius $r_{0}$ at $P \in M$, if there is a normal coordinate system $\left(y^{1}, \ldots, y^{\bar{n}}\right)$ of $\bar{M}$ around $P$ with $T_{P} M=\operatorname{span}\left\{\left(\partial / \partial y^{1}\right), \ldots,\left(\partial / \partial y^{n}\right)\right\}$, a vector valued function $F:\left\{y^{\prime}=\left(y^{1}, \ldots, y^{n}\right) \mid\left(y^{1}\right)^{2}+\cdots+\left(y^{n}\right)^{2}<r_{0}^{2}\right\} \rightarrow \mathbb{R}^{\bar{n}-n}$ with $F(0)=$ $0, \quad|D F|(0)=0$ such that $M \cap\left\{\left|y^{\prime}\right|<r_{0}\right\}=\left\{\left(y^{\prime}, F\left(y^{\prime}\right)\right)|| y^{\prime} \mid<r_{0}\right\}$ and $|D F|^{2}\left(y^{\prime}\right)=\sum_{i, \beta}\left(\partial F^{\beta} / \partial y^{i}\right)\left(\partial F^{\beta} / \partial y^{i}\right)<\delta^{2}$. The submanifold $M_{0}$ is said to be graphic in the ball $B_{\bar{M}}\left(x_{0}, r_{0}\right)$, if the above holds for $\delta=\infty$.

We say a submanifold $M \subset \bar{M}$ is properly embedded in a ball $B_{\bar{M}}\left(x_{0}, r_{0}\right)$ if either $M$ is closed or $\partial M$ has distance $\geq r_{0}$ from $x_{0}$. We say a submanifold $M \subset \bar{M}$ is properly embedded in $\bar{M}$ if either $M$ is closed or there is an $x_{0} \in \bar{M}$ such that $M$ is properly embedded in $B_{\bar{M}}\left(x_{0}, r_{0}\right)$ for any $r_{0}>0$. It is clear that if $\bar{M}$ is complete and $M$ is properly embedded in $\bar{M}$, then $M$ is complete. A properly embedded submanifold $M$ is said to be uniform graphic with radius $r_{0}$ if for any $x_{0} \in M$ it is graphic in the ball $B_{\bar{M}}\left(x_{0}, r_{0}\right)$.

The following lemma says that if the second fundamental form is controlled, then (a piece of) the submanifold is a local $\delta$-Lipschitz graph of suitable radius. 
Uniqueness and pseudolocality theorems of the mean curvature flow 475

Lemma 7.1. Let $\bar{M}$ be an $\bar{n}$-dimensional complete Riemannian manifold satisfying

$$
|\overline{\mathrm{R}} \mathrm{m}|+|\bar{\nabla} \overline{\mathrm{R}} \mathrm{m}|(x) \leq \bar{C}, \quad \operatorname{inj}(\bar{M}) \geq i_{0}>0 .
$$

There exists a constant $C_{1}>0$ with the following property. Let $\left\{x^{1}, \ldots, x^{\bar{n}}\right\}$ be normal coordinates of $\bar{M}$ of radius $r_{0}$ around $x_{0}$ with $T_{x_{0}} M=$ span $\left\{\left(\partial / \partial x^{1}\right), \ldots,\left(\partial / \partial x^{n}\right)\right\}$, where $M$ is an n-dimensional submanifold properly embedded in $B_{\bar{M}}\left(x_{0}, r_{0}\right), x_{0} \in M, r_{0} \leq\left(1 / C_{1}\right)$, and the second fundamental form $|A| \leq\left(1 / r_{0}\right)$. Then there exists a map $F:\left\{\left(x^{1}, \ldots, x^{n}\right) \mid\left(x^{1^{2}}+\right.\right.$ $\left.\left.\cdots+x^{n 2}\right)^{1 / 2}<\left(r_{0} / 96\right)\right\} \rightarrow \mathbb{R}^{\bar{n}-n}$ with $F(0)=0,|D F|(0)=0$ such that the connected component containing $x_{0}$ of $M \cap\left\{\left(x^{1}, \ldots, x^{\bar{n}}\right) \mid\left(x^{1^{2}}+\cdots+\right.\right.$ $\left.\left.x^{n 2}\right)^{1 / 2}<\left(r_{0} / 96\right)\right\}$ can be written as a graph $\left\{\left(x^{\prime}, F\left(x^{\prime}\right)\right)|| x^{\prime} \mid=\left(x^{1^{2}}+\cdots+\right.\right.$ $\left.\left.x^{n 2}\right)^{1 / 2}<\left(r_{0} / 96\right)\right\}$ and

$$
|D F|\left(x^{\prime}\right) \leq \frac{36}{r_{0}}\left|x^{\prime}\right|
$$

$x^{\prime}=\left(x^{1}, \ldots, x^{n}\right) \in B_{\mathbb{R}^{n}}\left(0,\left(r_{0} / 96\right)\right)$, where $|D F|\left(x^{\prime}\right)^{2}=\sum_{i=1}^{n} \sum_{\alpha=n+1}^{\bar{n}}\left(\partial F^{\alpha} /\right.$ $\left.\partial x^{i}\right)\left(\partial F^{\alpha} / \partial x^{i}\right)\left(x^{\prime}\right)$.

Proof. Let $X=\left(X^{1}, \ldots, X^{\bar{n}}\right)=\left(x^{\prime}, F\left(x^{\prime}\right)\right), x^{\prime}=\left(x^{1}, \ldots, x^{n}\right)$, be a graph representation of the local isometric embedding of the connected component containing $x_{0}$ of $M \cap\left\{\left(x^{1}, \ldots, x^{\bar{n}}\right) \mid\left(x^{1^{2}}+\cdots+x^{n 2}\right)^{1 / 2}<r_{1}\right\}$ (for some $\left.r_{1} \leq\left(r_{0} / 96\right)\right)$ into $\bar{M}$ under the exponential map.

Define

$$
|\nabla F|^{2}=\sum_{i, j=1}^{n} \sum_{\alpha=n+1}^{\bar{n}} \frac{\partial F^{\alpha}}{\partial x^{i}} \frac{\partial F^{\alpha}}{\partial x^{j}} g^{i j}, \quad|D F|^{2}=\sum_{i=1}^{n} \sum_{\alpha=n+1}^{\bar{n}} \frac{\partial F^{\alpha}}{\partial x^{i}} \frac{\partial F^{\alpha}}{\partial x^{i}} .
$$

By choosing $C_{1}$ large, we have

$$
\frac{1}{2} \delta_{\alpha \beta} \leq \bar{g}_{\alpha \beta} \leq 2 \delta_{\alpha \beta}, \quad\left|\bar{\Gamma}_{\alpha \beta}^{\gamma}\right| \leq 1, \quad \frac{1}{2} \delta_{i j} \leq g_{i j} \leq 2\left(1+|D F|^{2}\right) \delta_{i j} .
$$

For $\alpha \geq n+1, i, j \leq n$, recall the coefficients of the second fundamental form is given by

$$
A_{i j}^{\alpha}=\frac{\partial X^{\alpha}}{\partial x^{i} \partial x^{j}}-\Gamma_{i j}^{k} \frac{\partial X^{\alpha}}{\partial x^{k}}+\bar{\Gamma}_{\beta \gamma}^{\alpha} \frac{\partial X^{\beta}}{\partial x^{i}} \frac{\partial X^{\gamma}}{\partial x^{j}}=\nabla_{i j}^{2} F^{\alpha}+\bar{\Gamma}_{\beta \gamma}^{\alpha} \frac{\partial X^{\beta}}{\partial x^{i}} \frac{\partial X^{\gamma}}{\partial x^{j}} .
$$


Note that

$$
\begin{aligned}
\left|\bar{\Gamma}_{\beta \gamma}^{\alpha} \frac{\partial X^{\beta}}{\partial x^{i}} \frac{\partial X^{\gamma}}{\partial x^{j}}\right|^{2} & =\bar{\Gamma}_{\beta^{\prime} \gamma^{\prime}}^{\alpha^{\prime}} \frac{\partial X^{\beta^{\prime}}}{\partial x^{i}} \frac{\partial X^{\gamma^{\prime}}}{\partial x^{j}} \bar{\Gamma}_{\beta \gamma}^{\alpha} \frac{\partial X^{\beta}}{\partial x^{k}} \frac{\partial X^{\gamma}}{\partial x^{l}} g^{i k} g^{j l} \bar{g}_{\alpha \alpha^{\prime}} \leq C(\bar{n}) \\
\left|\nabla^{2} F\right|^{2} & =\sum_{\alpha, \beta \geq n+1 ; i, j, k, l \leq n} \nabla_{i j}^{2} F^{\alpha} \nabla_{k l}^{2} F^{\beta} \delta_{\alpha \beta} g^{i k} g^{j l} \\
& \leq 4\left(|A|^{2}+C(\bar{n})\right) \\
& \leq 4 r_{0}^{-2}+C(\bar{n})
\end{aligned}
$$

and

$$
|\nabla| \nabla F|| \leq\left|\nabla^{2} F\right|
$$

This implies

$$
|\nabla F|(\cdot) \leq 3 r_{0}^{-1} d_{M}\left(x_{0}, \cdot\right)
$$

Since $g_{i j} \leq 2\left(\delta_{i j}+\frac{\partial F^{\alpha}}{\partial x^{i}} \frac{\partial F^{\alpha}}{\partial x^{j}}\right) \leq 2\left(1+|D F|^{2}\right) \delta_{i j}$, it follows that

$$
|\nabla F|^{2} \geq \frac{1}{4} \frac{|D F|^{2}}{1+|D F|^{2}}
$$

and

$$
|D F|^{2} \leq \frac{4|\nabla F|^{2}}{1-4|\nabla F|^{2}}
$$

Combining (7.2)and (7.3), it follows that

$$
|D F|(\cdot) \leq 9 r_{0}^{-1} d_{M}\left(x_{0}, \cdot\right) \text { on } B_{M}\left(x_{0}, \frac{r_{0}}{24}\right) .
$$

Since $d_{M}\left(x_{0}, \cdot\right) \leq 2 d_{\bar{M}}\left(x_{0}, \cdot\right)$ by $(2.5)$, we have

$$
|D F|(\cdot) \leq 18 r_{0}^{-1} \sup _{B_{M}\left(0,\left(r_{0} / 24\right)\right)}(1+|D F|)\left|x^{\prime}\right| \leq 36 r_{0}^{-1}\left|x^{\prime}\right|,
$$

and we conclude that

$$
|D F|\left(x^{\prime}\right) \leq 36 r_{0}^{-1}\left|x^{\prime}\right|, \text { whenever }\left|x^{\prime}\right| \leq \frac{r_{0}}{96} .
$$

The above argument shows that there is $C_{1}>0$ such that under the exponential map, once the connected component of $M$ can be expressed as a 
graph $\left(x^{\prime}, F\left(x^{\prime}\right)\right)$ on $B_{\mathbb{R}^{n}}\left(0, r_{1}\right)$, for $r_{1} \leq\left(r_{0} / 96\right)$, then the estimate $(7.1)$ holds. Hence the connected component of $M$ can be expressed as a graph on the ball $B_{\mathbb{R}^{n}}\left(0,\left(r_{0} / 96\right)\right)$.

For future applications in pseudolocality theorem, we need a local graph representation for mean curvature flow.

Lemma 7.2. Fix $k \geq 1$. Let $\bar{M}$ be an $\bar{n}$-dimensional complete manifold satisfying

$$
\sum_{i=0}^{k+1}\left|\bar{\nabla}^{i} \overline{\operatorname{R}} \mathrm{m}\right|(x) \leq \bar{C}, \quad \operatorname{inj}(\bar{M}) \geq i_{0}>0 .
$$

There exists a constant $C_{1}>0$ with the following property. Suppose $M_{s}$, $s \in\left[-r_{0}^{2}, 0\right]$ is a solution of MCF properly embedded in $B_{\bar{M}}\left(x_{0}, r_{0}\right), x_{0} \in M_{0}$, $r_{0} \leq\left(1 / C_{1}\right)$, with $\sum_{i=0}^{k}\left|\nabla^{i} A\right| r_{0}^{i+1} \leq 1$ on $B_{\bar{M}}\left(x_{0}, r_{0}\right)$. Denote by $x_{0}^{s} \in M_{s}$ the orbit of $x_{0}$. Let $\left\{x^{1}, \ldots, x^{\bar{n}}\right\}$ be normal coordinates of $\bar{M}$ of radius $r_{0}$ around $x_{0}$ with $T_{x_{0}} M_{0}=\operatorname{span}\left\{\left(\partial / \partial x^{1}\right), \ldots,\left(\partial / \partial x^{n}\right)\right\}$. Then there exist $a$ family of smooth maps $F_{s}:\left\{\left(x^{1}, \ldots, x^{n}\right) \mid\left(x^{1^{2}}+\cdots+x^{n 2}\right)^{1 / 2}<\left(r_{0} / C_{1}\right)\right\} \rightarrow$ $\mathbb{R}^{\bar{n}-n}$ with $F_{0}(0)=0,\left|D_{0} F\right|(0)=0$, e $\bar{x} p_{x_{0}}\left(\left(0, F_{s}(0)\right)\right)=x_{0}^{s}$ such that the connected component of $M_{s} \cap\left\{\left(x^{1}, \ldots, x^{\bar{n}}\right) \mid\left(x^{1^{2}}+\cdots+x^{n 2}\right)^{1 / 2}<\left(r_{0} /\right.\right.$ $\left.\left.C_{1}\right)\right\}$ (under the exponential map $\operatorname{ex}_{x_{0}}$ ) containing $x_{0}^{s}$ can be written as a graph $\left\{\left(x^{\prime}, F_{s}\left(x^{\prime}\right)\right)|| x^{\prime} \mid=\left(x^{1^{2}}+\cdots+x^{n 2}\right)^{1 / 2}<\left(r_{0} / C_{1}\right)\right\} ;$ moreover we have $\sum_{i=1}^{k+2} r_{0}^{i+1}\left|D^{i} F_{s}\right| \leq C_{1}$.

Actually, by the MCF equation $(\partial / \partial s) X=\triangle X$, where $X=\left(x^{\prime}, F_{s}\left(x^{\prime}\right)\right)$ is the graph representations on $B\left(0, r_{1}\right)$ for some $r_{1}<\left(r_{0} / C_{1}\right)$, we have information on $\left|(\partial / \partial s) F_{s}\right| r_{0}+\left|(\partial / \partial s) D F_{s}\right| r_{0}^{2} \leq C_{1}$. This gives $\left|F_{s}(0)\right| \leq C s r_{0}^{-1}$ and $\left|D F_{s}\right|(0) \leq C s r_{0}^{-2}$. Similarly, by integrating $|\nabla| \nabla F|| \leq\left|\nabla^{2} F\right|$, we know the graph representation holds in a ball of uniform radius $\left(r_{1} / C_{1}\right)$. The higher-derivative $D^{i} F$ can be estimated by $\sum_{j \leq i}\left|\nabla^{j} F\right|$ by definitions.

Now we state the pseudolocality theorem for the MCF.

Theorem 7.3. Let $\bar{M}$ be an $\bar{n}$-dimensional complete manifold satisfying $\sum_{i=0}^{3}\left|\bar{\nabla}^{i} \overline{\mathrm{R}} \mathrm{m}\right| \leq c_{0}^{2}$ and $\operatorname{inj}(\bar{M}) \geq i_{0}>0$. Then for every $\alpha>0$ there exist $\varepsilon>0, \delta>0$ with the following property. Suppose we have a smooth solution to the mean curvature flow $M_{t} \subset \bar{M}$ properly embedded in $B_{\bar{M}}\left(x_{0}, r_{0}\right)$ for $t \in[0, T]$ with $0<T \leq \varepsilon^{2} r_{0}^{2}$, and assume that at time zero, $M_{0}$ is a local $\delta$-Lipschitz graph of radius $r_{0}$ at $x_{0} \in M_{0}$ with $r_{0} \leq\left(i_{0} / 2\right)$. Then we have 
an estimate of the second fundamental form

$$
|A|(x, t)^{2} \leq \frac{\alpha}{t}+\left(\varepsilon r_{0}\right)^{-2}
$$

on $B_{\bar{M}}\left(x_{0}, \varepsilon r_{0}\right) \cap M_{t}$, for any $t \in[0, T]$.

Proof. We argue by contradiction. By scaling we may assume $r_{0}=1$. Suppose there exist fixed $c_{0}>0, i_{0}>0, \alpha>0$, and a sequence of $\varepsilon, \delta \rightarrow 0$ and smooth solutions to the mean curvature flow $M_{t} \subset \bar{M}$ for $t \in[0, T] \subseteq\left[0, \varepsilon^{2}\right]$ such that at time zero, $M_{0}$ is a local $\delta$-Lipschitz graph of radius 1 at $x_{0} \in M$. But there is some $\left(x_{1}, t_{1}\right)$ satisfying $0 \leq t_{1} \leq T$ and $x_{1} \in B_{\bar{M}}\left(x_{0}, \varepsilon\right)$ such that

$$
|A|\left(x_{1}, t_{1}\right)^{2}>\frac{\alpha}{t_{1}}+\varepsilon^{-2} .
$$

Denote by $E_{\alpha}$ the set of points $(x, t)$ satisfying $|A|(x, t)^{2} \geq(\alpha / t)$. Now we use the Perelman's point-picking technique [20] to choose another point which controls nearby points in its scale.

Lemma 7.4. For any $K>0$ with $K \varepsilon<(1 / 100 n)$, let $M_{t}$ be assumed as in the theorem, suppose $|A|\left(x_{1}, t_{1}\right)^{2} \geq\left(\alpha / t_{1}\right)+\varepsilon^{-2}$ for some $\left(x_{1}, t_{1}\right)$ satisfying $0 \leq t_{1} \leq T \leq \varepsilon^{2}$ and $x_{1} \in B_{\bar{M}}\left(x_{0}, \varepsilon\right)$, then one can find $(\bar{x}, \bar{t}) \in E_{\alpha}$ with $0<\bar{t} \leq T, d_{\bar{M}}\left(x_{0}, \bar{x}\right) \leq(2 K+1) \varepsilon$ such that

$$
|A|(x, t) \leq 4 Q
$$

whenever $\bar{t}-(3 / 4) \alpha Q^{-2} \leq t \leq \bar{t}, d_{\bar{M}}(x, \bar{x}) \leq K Q^{-1}$, where $Q=|A|(\bar{x}, \bar{t})$.

Firstly, we claim that there exists $(\bar{x}, \bar{t}) \in E_{\alpha}$ with $0<\bar{t} \leq T, d_{\bar{M}}\left(x_{0}, \bar{x}\right) \leq$ $(2 K+1) \varepsilon$ such that

$$
|A|(x, t) \leq 4|A|(\bar{x}, \bar{t})
$$

whenever $(x, t) \in E_{\alpha}, 0 \leq t \leq \bar{t}, d_{\bar{M}}\left(x_{0}, x\right) \leq d_{\bar{M}}\left(x_{0}, \bar{x}\right)+K|A|(\bar{x}, \bar{t})^{-1}$.

The argument is by contradiction. If $\left(x_{1}, t_{1}\right)$ can not be chosen for $(\bar{x}, \bar{t})$, one can find $\left(x_{2}, t_{2}\right) \in E_{\alpha}$ with $0 \leq t_{2} \leq t_{1}, d_{\bar{M}}\left(x_{0}, x_{2}\right) \leq d_{\bar{M}}\left(x_{0}, x_{1}\right)+K|A|$ $\left(x_{1}, t_{1}\right)^{-1},|A|\left(x_{2}, t_{2}\right)>4|A|\left(x_{1}, t_{1}\right)$. Inductively, we have a sequence of $\left(x_{k}, t_{k}\right) \in E_{\alpha}$ with $0 \leq t_{k} \leq t_{k-1}, d_{\bar{M}}\left(x_{0}, x_{k}\right) \leq d_{\bar{M}}\left(x_{0}, x_{k-1}\right)+K|A|\left(x_{k-1}\right.$, $\left.t_{k-1}\right)^{-1},|A|\left(x_{k}, t_{k}\right)>4|A|\left(x_{k-1}, t_{k-1}\right)$. Therefore, we have

$$
|A|\left(x_{k}, t_{k}\right)>4^{k-1}|A|\left(x_{1}, t_{1}\right) \geq 4^{k-1} \varepsilon^{-1}
$$

and $\quad d_{\bar{M}}\left(x_{0}, x_{k}\right) \leq d_{\bar{M}}\left(x_{0}, x_{1}\right)+K \sum_{i=1}^{\infty}\left(4^{i-1}|A|\left(x_{1}, t_{1}\right)\right)^{-1} \leq(2 K+1) \varepsilon<$ $1 / 2$. Since the solution is smooth, we get a contradiction as $k$ large enough. 
Uniqueness and pseudolocality theorems of the mean curvature flow 479

For the chosen $(\bar{x}, \bar{t})$, if $(x, t) \notin E_{\alpha}, \bar{t}-(3 / 4) \alpha Q^{-2} \leq t \leq \bar{t}$, then

$$
|A|^{2}(x, t) \leq \frac{\alpha}{t} \leq \frac{\alpha}{\bar{t}-(3 / 4) \alpha Q^{-2}} \leq 4 Q^{2}
$$

If $(x, t) \in E_{\alpha}$ and $d_{\bar{M}}(x, \bar{x}) \leq K|A|(\bar{x}, \bar{t})^{-1}$, by above claim we still have the estimate. The lemma is proved.

Continuing the proof of Theorem 7.3.

Choose $K=(1 / \sqrt{\varepsilon})$. Let $(\bar{x}, \bar{t})$ be the point obtained in Lemma 7.4. Consider the auxiliary functions

$$
\varphi(x, t)=(4 \pi(\bar{t}-t))^{-(n / 2)} \exp \left[-\left(1+\frac{1}{\varepsilon}(t-\bar{t})\right) \frac{d_{\bar{M}}^{2}(\bar{x}, x)}{4(\bar{t}-t)}-\frac{n}{2 \varepsilon} t\right],
$$

and

$$
\psi(x, t)=\left(1-\frac{d_{\bar{M}}(\bar{x}, x)^{2}+3 n t}{\rho^{2}}\right)_{+}^{3}
$$

on $\bar{M} \times[0, \bar{t}]$, where $\rho=\min \left\{(1 / 2),\left(1 / c_{0} \sqrt{e}\right), i_{0}, \sqrt{\varepsilon}\right\}$. They are also functions on $M$ by composing the inclusion maps. We will compute their equations on $M$. Since the sectional curvature of $\bar{M}$ satisfies $-c_{0}^{2} \leq \sec \leq c_{0}^{2}$, by comparison theorem and mean curvature flow equation, we have

$$
\begin{aligned}
\left(\frac{\partial}{\partial t}+\triangle\right) d_{\bar{M}}(\bar{x}, \cdot)^{2} & =4 d_{\bar{M}} \bar{\nabla} d_{\bar{M}} \cdot H+\operatorname{tr}\left(\left.\operatorname{Hess}\left(d_{\bar{M}}^{2}(\bar{x}, \cdot)\right)\right|_{T M}\right) \\
& \geq 4 d_{\bar{M}} \bar{\nabla} d_{\bar{M}} \cdot H+2 n \frac{c_{0} d_{\bar{M}}(\bar{x}, \cdot)}{\tan c_{0} d_{\bar{M}}(\bar{x}, \cdot)} \\
& \geq 4 d_{\bar{M}} \bar{\nabla} d_{\bar{M}} \cdot H+2 n\left(1-\frac{1}{2} c_{0}^{2} d_{\bar{M}}^{2}(\bar{x}, \cdot)\right), \\
\left(\frac{\partial}{\partial t}-\triangle\right) d_{\bar{M}}(\bar{x}, \cdot)^{2} & =-\operatorname{tr}\left(\left.\operatorname{Hess}\left(d_{\bar{M}}^{2}(\bar{x}, \cdot)\right)\right|_{T M}\right) \\
& \geq-2 n c_{0} d_{\bar{M}}(\bar{x}, \cdot) \operatorname{coth}\left(c_{0} d_{\bar{M}}(\bar{x}, \cdot)\right) \geq-3 n
\end{aligned}
$$

whenever $d_{\bar{M}}(\bar{x}, \cdot)^{2}<\min \left\{\left(1 / c_{0}^{2} e\right), i_{0}^{2}\right\}, t \in[0, \bar{t}]$. Hence we have

$$
\left(\frac{\partial}{\partial t}-\triangle\right) \psi \leq 0
$$


and

$$
\begin{aligned}
& \left(\frac{\partial}{\partial t}+\triangle-|H|^{2}\right) \varphi=\varphi\left[\frac{n}{2(\bar{t}-t)}-\frac{1+(1 / \varepsilon)(t-\bar{t})}{4(\bar{t}-t)}\left(\frac{\partial}{\partial t}+\triangle\right) d_{\bar{M}}(\bar{x}, \cdot)^{2}\right. \\
& -\frac{(1+(1 / \varepsilon)(t-\bar{t})) d_{\bar{M}}(\bar{x}, \cdot)^{2}}{4(\bar{t}-t)^{2}} \\
& +\frac{(1+(1 / \varepsilon)(t-\bar{t}))^{2}\left|\nabla d_{\bar{M}}(\bar{x}, \cdot)^{2}\right|^{2}}{16(\bar{t}-t)^{2}} \\
& \left.-\frac{(1 / \varepsilon) d_{\bar{M}}(\bar{x}, \cdot)^{2}}{4(\bar{t}-t)}-\frac{n}{2 \varepsilon}-|H|^{2}\right] \\
& \leq \varphi\left[-\frac{1+(1 / \varepsilon)(t-\bar{t})}{(\bar{t}-t)} d_{\bar{M}} \bar{\nabla} d_{\bar{M}} \cdot H\right. \\
& -\frac{(1+(1 / \varepsilon)(t-\bar{t})) d_{\bar{M}}(\bar{x}, \cdot)^{2}}{4(\bar{t}-t)^{2}} \\
& +\frac{(1+(1 / \varepsilon)(t-\bar{t}))^{2}\left|\nabla d_{\bar{M}}(\bar{x}, \cdot)^{2}\right|^{2}}{16(\bar{t}-t)^{2}} \\
& \left.-\frac{\left[(1 / \varepsilon)-(1+(1 / \varepsilon)(t-\bar{t})) n c_{0}^{2}\right] d_{\bar{M}}(\bar{x}, \cdot)^{2}}{4(\bar{t}-t)}-|H|^{2}\right] \\
& \leq-\left|H+\left(1+\frac{1}{\varepsilon}(t-\bar{t})\right) \frac{d_{\bar{M}}(\bar{x}, \cdot) \bar{\nabla}^{\perp} d_{\bar{M}}(\bar{x}, \cdot)}{2(\bar{t}-t)}\right|^{2} \varphi
\end{aligned}
$$

whenever $d_{\bar{M}}(\bar{x}, \cdot)<\rho, t \in[0, \bar{t}]$. We used $0<1+(1 / \varepsilon)(t-\bar{t}) \leq 1$. In the above and following argument, we regard the mean curvature flow $M_{t}$ is a smooth family of $F_{t}: M \rightarrow \bar{M},(\varphi \psi) \circ F_{t}$ is a $C^{2}$ function on $M \times[0, \bar{t}]$ with compact support in $M$. So $\int_{M_{t}} \varphi \psi=\int_{M} \varphi \psi d v_{t}$ is a $C^{2}$ function in $t$. Combining (7.6) and (7.7), we get the monotonicity formula

$$
\frac{d}{d t} \int_{M_{t}} \varphi \psi \leq-\int_{M_{t}}\left|H+\left(1+\frac{1}{\varepsilon}(t-\bar{t})\right) \frac{d_{\bar{M}}(\bar{x}, \cdot) \bar{\nabla}^{\perp} d_{\bar{M}}(\bar{x}, \cdot)}{2(\bar{t}-t)}\right|^{2} \varphi \psi
$$

on $[0, \bar{t}]$. This implies

$$
\begin{aligned}
& \int_{\bar{t}-(1 / 2) \alpha Q^{-2}}^{\bar{t}}\left[\int_{M_{t}}\left|H+\left(1+\frac{1}{\varepsilon}(t-\bar{t})\right) \frac{d_{\bar{M}}(\bar{x}, \cdot) \overline{\nabla^{\perp}} d_{\bar{M}}(\bar{x}, \cdot)}{2(\bar{t}-t)}\right|^{2} \varphi \psi\right] d t \\
& (7.9) \leq \int_{M_{\bar{t}-(1 / 2) \alpha Q^{-2}}} \varphi \psi-\int_{M_{\bar{t}}} \varphi \psi .
\end{aligned}
$$


Since the solution is smooth and properly embedded, $\psi$ is compactly sup-

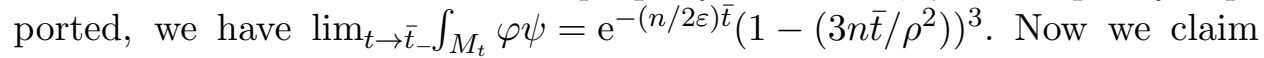
that there is $\beta>0$ such that as $\varepsilon, \delta \rightarrow 0$, we have

$$
\int_{M_{\bar{t}-(1 / 2) \alpha Q^{-2}}} \varphi \psi \geq(1+\beta) \mathrm{e}^{-(n / 2 \varepsilon) \bar{t}}\left(1-\frac{3 n \bar{t}}{\rho^{2}}\right)^{3} .
$$

We still argue by contradiction. Suppose not, then there is a subsequence of $\varepsilon, \delta \rightarrow 0$ and

$$
\begin{aligned}
& \int_{\bar{t}-(1 / 2) \alpha Q^{-2}}^{\bar{t}}\left[\int_{M_{t}}\left|H+\left(1+\frac{1}{\varepsilon}(t-\bar{t})\right) \frac{d_{\bar{M}}(\bar{x}, \cdot) \bar{\nabla}^{\perp} d_{\bar{M}}(\bar{x}, \cdot)}{2(\bar{t}-t)}\right|^{2} \varphi \psi d v\right] d t \\
& \quad \leq \beta \rightarrow 0 .
\end{aligned}
$$

Parabolic scaling the solution around $(\bar{x}, \bar{t})$ with the factor $Q$ and shifting the $\bar{t}$ to 0 and $\bar{x}$ to origin $O$, i.e., let $(\tilde{M}, \tilde{g})=\left(\bar{M}, Q^{2} \bar{g}\right)$ be the new target manifold, $\tilde{M}_{s}=M_{\bar{t}+Q^{-2} s},-(3 / 4) \alpha \leq s \leq 0$ be the new family of submanifolds, which is still solution of MCF. By (7.5), the normalized second fundamental form satisfies $|\tilde{A}| \leq 4$ on $B_{\tilde{M}}(\bar{x}, K),-(3 / 4) \alpha \leq s \leq 0$. By Theorem 3.2, we have $|\tilde{\nabla} \tilde{A}|+\left|\tilde{\nabla}^{2} \tilde{A}\right| \leq$ Const. on $B_{\tilde{M}}(\bar{x},(K / 2)),-(5 / 8) \alpha \leq s \leq 0$. Note that $K \rightarrow \infty$.

Now we are going to consider the convergence of the MCF on changing target manifolds. We clarify the meaning of the convergence in the following.

Denote the orbit of $\bar{x}$ under MCF by $\bar{x}^{s} \in \tilde{M}_{s}$ such that $\bar{x}^{0}=\bar{x}$. Note the injectivity radius of the new target $(\tilde{M}, \tilde{g})$ tends to infinity as $\varepsilon \rightarrow 0$. Let $\left\{x^{1}, \ldots, x^{\bar{n}}\right\}$ be normal coordinates of $\tilde{M}$ of radius $\gg 1$ around $\bar{x}$ with $T_{\bar{x}} \tilde{M}_{0}=\operatorname{span}\left\{\left(\partial / \partial x^{1}\right), \ldots,\left(\partial / \partial x^{n}\right)\right\}$, and $\tilde{g}_{\alpha \beta}$ be the metric coefficients of $\tilde{M}$ in this coordinates. By [14], we have $\left|\tilde{g}_{\alpha \beta}-\delta_{\alpha \beta}\right|(x) \leq C Q^{-2}|x|^{2}$ and $\left|\partial \tilde{g}_{\alpha \beta}\right|+\left|\partial^{2} \tilde{g}_{\alpha \beta}\right| \leq C$. By Arzela-Ascoli theorem, after taking a subsequence of $\varepsilon \rightarrow 0, \tilde{g}_{\alpha \beta}$ tends to $\delta_{\alpha \beta}$ in $C^{2-\gamma}$ topology for any $0<\gamma<1$.

By Lemma 7.2, there exist a family of maps $F_{s}:\left\{\left(x^{1}, \ldots, x^{n}\right) \mid\right.$ $\left.\left(x^{1^{2}}+\cdots+x^{n 2}\right)^{1 / 2}<1\right\} \rightarrow \mathbb{R}^{\bar{n}-n}$ with $F_{0}(0)=0,\left|D F_{0}\right|(0)=0$, such that the connected component containing $\bar{x}^{s}$ of $\tilde{M}_{s} \cap\left\{\left(x^{1}, \ldots, x^{\bar{n}}\right) \mid\left(x^{1^{2}}+\cdots+\right.\right.$ $\left.\left.x^{n 2}\right)^{1 / 2}<1\right\}$ can be written as a graph $\left\{\left(x^{\prime}, F_{s}\left(x^{\prime}\right)\right)|| x^{\prime} \mid=\left(x^{1^{2}}+\cdots+\right.\right.$ $\left.\left.x^{n 2}\right)^{1 / 2}<1\right\}$. Moreover, we can show

$$
\sum_{i=1}^{4}\left|D^{i} F\right|+\sum_{i=1}^{2}\left(\left|\frac{\partial^{i}}{\partial s^{i}} F\right|+\left|D^{i} \frac{\partial F}{\partial s}\right|\right) \leq C,
$$


where $D$ and the norm are the natural differential and norm in Euclidean ordinates of $N \subset \mathbb{R}^{n}$ and the garget $\mathbb{R}^{\bar{n}}$. By Arzela-Ascoli theorem, $F\left(x^{\prime}, s\right)$ will converge to $F^{\infty}\left(x^{\prime}, s\right)$ in the topology of $\mathcal{C}^{3 / 2}(\overline{B(0,(1 / 2))} \times[-(5 \alpha / 8), 0]$, $\left.\mathbb{R}^{\bar{n}}\right) \cap \mathcal{C}^{3}\left(\overline{B(0,(1 / 2))}, \mathbb{R}^{\bar{n}}\right)$.

If we set $X=\left(x^{\prime}, F\left(x^{\prime}\right)\right)$ being the map from $N:=B(0,1)$ to $\tilde{M}$, then the MCF equation can be written as

$$
\frac{\partial X}{\partial s}=\triangle X
$$

where $\triangle$ is the harmonic Laplacian defined by using the induced metric $X^{*} \tilde{g}$ and the target metric $\tilde{g}$. Since $X^{*} \tilde{g}$ is defined by $D F$ and $\tilde{g}$, after taking a subsequence of $\varepsilon \rightarrow 0$, we know $X^{*} \tilde{g}$ converges in $\mathcal{C}^{1-\gamma}(\overline{B(0,(1 / 2))} \times$ $[-(5 \alpha / 8), 0])$ topology.

Denote by $\hat{M}_{s}=\tilde{M}_{s} \cap \exp _{\bar{x}}\left\{\left|x^{\prime}\right|<1\right\}$, and $\hat{M}=\cup_{s \in[-(\alpha / 2), 0]} \hat{M}_{s}$. By summing up the above discussion, the piece $\hat{M}$ of $\tilde{M}$ containing $(\bar{x}, 0)$ will converge to a solution of the MCF (in the classical sense) which is embedded on the Euclidean space $\mathbb{R}^{\bar{n}}$ with $\left|\hat{A}_{\infty}\right|(O, 0)=1$ and $\left|\hat{A}_{\infty}\right|(\cdot, s) \leq 4$ on $[-(\alpha / 2), 0]$.

On the other hand, let $\tilde{\varphi}=Q^{-n} \varphi=(4 \pi(-s))^{-(n / 2)} \exp \left[-\left(1+\left(s / Q^{2} \varepsilon\right)\right)\right.$ $\left.\left(d_{\tilde{M}}^{2}(\bar{x}, \cdot) / 4(-s)\right)-(n / 2 \varepsilon)\left(\bar{t}+Q^{-2} s\right)\right]$, note that

$$
\begin{aligned}
Q^{-2}\left|H+\left(1+\frac{1}{\varepsilon}(t-\bar{t})\right) \frac{d_{\bar{M}}(\bar{x}, \cdot) \bar{\nabla}{ }^{\perp} d_{\bar{M}}(\bar{x}, \cdot)}{2(\bar{t}-t)}\right|_{\bar{g}}^{2} \\
=\left|\tilde{H}-\left(1+\frac{s}{Q^{2} \varepsilon}\right) \frac{d_{\tilde{M}}(\bar{x}, \cdot) \tilde{\nabla}^{\perp} d_{\tilde{M}}(\bar{x}, \cdot)}{2 s}\right|_{\tilde{g}}^{2}, \\
\psi=\left(1-\frac{Q^{-2} d_{\tilde{M}}(\bar{x}, \cdot)^{2}+3 n \bar{t}+3 n Q^{-2} s}{\rho^{2}}\right)_{+}^{3} \longrightarrow 1, \\
\tilde{\varphi} \rightarrow(4 \pi(-s))^{-(n / 2)} \mathrm{e}^{-\left(|\cdot|^{2}\right) / 4(-s)} \text { and } \varphi \psi d v=\tilde{\varphi} \psi d \tilde{v} .
\end{aligned}
$$

Since $\hat{M}_{s} \subset \tilde{M}_{s}$, by passing (7.11) to limit, we have

$$
\int_{-(1 / 2) \alpha}^{0}\left[\int_{\hat{M}_{s}^{\infty}}\left|\hat{H}_{\infty}-\frac{x^{\perp}}{2 s}\right|^{2}(4 \pi(-s))^{-(n / 2)} \mathrm{e}^{-\left(|x|^{2} / 4(-s)\right)}\right] d s=0
$$


Uniqueness and pseudolocality theorems of the mean curvature flow 483

where we denote the limit of $\hat{M}_{s}$ by $\hat{M}_{s}^{\infty}, \hat{H}_{\infty}$ the mean curvature on the limit. This implies

$$
\hat{H}_{\infty}=\frac{x^{\perp}}{2 s} \quad \text { for } s \in\left[-\frac{\alpha}{2}, 0\right] .
$$

The boundedness of the second fundamental form on $\hat{M}_{0}^{\infty}$ implies $x^{\perp} \equiv 0$ on $\hat{M}_{0}^{\infty}$. Since the second fundamental form and its twice covariant derivative of $\hat{M}_{s}^{\infty}$ are bounded for $s \in[-(\alpha / 2), 0], \hat{M}_{s}^{\infty}$ are $C^{4-\gamma}$ submanifolds for any $\gamma>0$. Moreover by the higher-derivative estimates in Theorem 3.2 (in Euclidean space), $\hat{M}_{0}^{\infty}$ is smooth.

Note $0 \in \hat{M}_{0}^{\infty}$, after a orthogonal transformation, we may assume $T_{0} \hat{M}_{0}^{\infty}$ $\left\{\left(x_{1}, x_{2}, \ldots, x_{n}, 0, \ldots, 0\right)\right\}$. Clearly we still have the condition $x^{\perp} \equiv 0$ on $\hat{M}_{0}^{\infty}$. We may write $\hat{M}_{0}^{\infty}$ as a graph (at least locally near 0$)\left(x^{\prime}, f_{1}\left(x^{\prime}\right), \ldots\right.$, $\left.f_{\bar{n}-n}\left(x^{\prime}\right)\right)$ where $x^{\prime}=\left(x_{1}, \ldots, x_{n}\right)$. Now $x^{\perp}=\left(x^{\prime}, f_{1}\left(x^{\prime}\right), \ldots, f_{\bar{n}-n}\left(x^{\prime}\right)\right)^{\perp} \equiv 0$ implies $\sum_{p=1}^{n}\left(\partial f_{i} / \partial x_{p}\right) x_{p}=f_{i}\left(x^{\prime}\right)$. So $f_{i}$ is homogenous of degree 1. Since $D f_{i}(0)=0$, we conclude $f_{i} \equiv 0$. Hence we know $\hat{M}_{0}^{\infty}$ is an $n$-dimensional linear subspace $\mathbb{R}^{n}$ of $\mathbb{R}^{\bar{n}}$.

This contradicts $\left|\hat{A}_{\infty}\right|(O, 0)=1$ and we complete the proof of (7.10).

Note that $B_{\bar{M}}(\bar{x}, \rho) \subseteq B_{\bar{M}}\left(x_{0}, \rho+(2 K+1) \varepsilon\right) \subseteq B_{\bar{M}}\left(x_{0}, 4 \sqrt{\varepsilon}\right)$. Combining (7.10) and monotonicity formula (7.8), we know

$$
\begin{aligned}
& \int_{M_{0} \cap B_{\bar{M}}\left(x_{0}, 4 \sqrt{\varepsilon}\right)}(4 \pi \bar{t})^{-(n / 2)} \exp \left[-\left(1-\frac{\bar{t}}{\varepsilon}\right) \frac{d_{\bar{M}}^{2}(\bar{x}, x)}{4 \bar{t}}\right] d v \\
& \geq\left.\int_{M_{t}} \varphi \psi d v\right|_{t=\bar{t}-(1 / 2) \alpha Q^{-2}} \geq(1+\beta) e^{-(n / 2 \varepsilon) \bar{t}}\left(1-\frac{3 n \bar{t}}{\rho^{2}}\right)^{3} .
\end{aligned}
$$

By assumption, there is a normal coordinate system $\left(y^{1}, \ldots, y^{\bar{n}}\right)$ of $\bar{M}$ around $x_{0}$ with $T_{x_{0}} M_{0}=\operatorname{span}\left\{\left(\partial / \partial y^{1}\right), \ldots,\left(\partial / \partial y^{n}\right)\right\}$ and a vector valued function $F:\left\{y^{\prime}=\left(y^{1}, \ldots, y^{n}\right) \mid\left(y^{1}\right)^{2}+\cdots+\left(y^{n}\right)^{2}<1\right\} \rightarrow \mathbb{R}^{\bar{n}-n} \quad$ with $\quad F(0)=0$, $|D F|(0)=0,|D F|^{2}\left(y^{\prime}\right)=\sum_{i, \gamma}\left(\partial F^{\gamma} / \partial y^{i}\right)\left(\partial F^{\gamma} / \partial y^{i}\right) \leq \delta^{2}$ such that $M_{0} \cap$ $\left\{\left|y^{\prime}\right|<1\right\}=\left\{\left(y^{\prime}, F\left(y^{\prime}\right)\right)|| y^{\prime} \mid<1\right\}$. Let $P: \mathbb{R}^{\bar{n}} \rightarrow \mathbb{R}^{n}$ be the orthogonal projection into the first $n$-components. Let $\exp _{x_{0}}(\bar{y})=\bar{x}$ and $\bar{y}^{\prime}=P \bar{y}$. For $x \in B_{\bar{M}}\left(x_{0}, 4 \sqrt{\varepsilon}\right)$, let $\exp _{x_{0}}(y)=x$ and $y^{\prime}=P y$. Since the curvature of $\bar{M}$ is bounded by $c_{0}^{2}$, by comparison theorem on the ball $B_{T_{x_{0}} \bar{M}}(o, 4 \sqrt{\varepsilon})$, we have

$$
d_{\bar{M}}(\bar{x}, x) \geq \frac{\sin \left(4 c_{0} \sqrt{\varepsilon}\right)}{4 c_{0} \sqrt{\varepsilon}}|\bar{y}-y| \geq\left(1-3 c_{0}^{2} \varepsilon\right)|\bar{y}-y| \geq\left(1-3 c_{0}^{2} \varepsilon\right)\left|\bar{y}^{\prime}-y^{\prime}\right| .
$$


On the other hand, also by comparison theorem, the Riemannian volume element $d v$ of $M_{0}$ satisfies

$$
\exp _{x_{0}}^{*} d v \leq\left[\frac{\sinh \left(c_{0} d_{\bar{M}}\left(x_{0}, \cdot\right)\right)}{c_{0} d_{\bar{M}}\left(x_{0}, \cdot\right)}\right]^{n} d v_{\exp _{x_{0}}^{-1} M_{0}} \leq\left[1+16 c_{0}^{2} \varepsilon\right]^{n} d v_{\exp _{x_{0}}^{-1} M_{0}}
$$

whenever $x \in M_{0} \cap B_{\bar{M}}\left(x_{0}, 4 \sqrt{\varepsilon}\right)$. By definition, it is clear that

$$
d v_{\exp _{x_{0}}^{-1} M_{0}} \leq\left(1+|D F|^{2}\right)^{n / 2} d y^{1} \ldots d y^{n} \leq\left(1+\delta^{2}\right)^{n / 2} d y^{1} \ldots d y^{n} .
$$

Combining (7.13), (7.14) and (7.15), we have

$$
\begin{aligned}
& \int_{M_{0} \cap B_{\bar{M}}\left(x_{0}, 4 \sqrt{\varepsilon}\right)}(4 \pi \bar{t})^{-(n / 2)} \exp \left[-\left(1-\frac{\bar{t}}{\varepsilon}\right) \frac{d_{\bar{M}}^{2}(\bar{x}, x)}{4 \bar{t}}\right] d v \\
& \leq\left(1+\delta^{2}\right)^{n / 2}\left(1+16 c_{0}^{2} \varepsilon\right)^{n}(1-\varepsilon)^{-(n / 2)}\left(1-3 c_{0}^{2} \varepsilon\right)^{-n} \\
& \times \int_{\left(\left|y^{1}\right|^{2}+\cdots+\left|y^{n}\right|^{2}\right)^{1 / 2}<4 \sqrt{\varepsilon}}\left[\frac{4 \pi \bar{t}}{(1-\varepsilon)\left(1-3 c_{0}^{2} \varepsilon\right)^{2}}\right]^{-(n / 2)} \\
& \times \exp \left[-\left|\bar{y}^{\prime}-y^{\prime}\right|^{2} / \frac{4 \bar{t}}{(1-\varepsilon)\left(1-3 c_{0}^{2} \varepsilon\right)^{2}}\right] d y^{1} \ldots d y^{n} \\
& \leq\left(1+\delta^{2}\right)^{n / 2}\left(1+16 c_{0}^{2} \varepsilon\right)^{n}(1-\varepsilon)^{-(n / 2)}\left(1-3 c_{0}^{2} \varepsilon\right)^{-n} \text {. }
\end{aligned}
$$

By (7.12) and the fact $\bar{t} \leq \varepsilon^{2}$, we conclude that

$\left(1+\delta^{2}\right)^{(n / 2)}\left(1+16 c_{0}^{2} \varepsilon\right)^{n}(1-\varepsilon)^{-(n / 2)}\left(1-3 c_{0}^{2} \varepsilon\right)^{-n}(1-3 n \varepsilon)^{-3} \mathrm{e}^{n \varepsilon / 2} \geq(1+\beta)$,

which is a contradiction as $\varepsilon, \delta \rightarrow 0$. We complete the proof of the Theorem.

Theorem 7.5. Let $\bar{M}$ be an $\bar{n}$-dimensional manifold satisfying $\sum_{i=0}^{3} \mid$ $\bar{\nabla}^{i} \overline{\mathrm{R}} \mathrm{m} \mid \leq c_{0}^{2}$ and $\operatorname{inj}(\bar{M}) \geq i_{0}>0$. Then there is $\varepsilon>0$ with the following property. Suppose we have a smooth solution $M_{t} \subset \bar{M}$ to the MCF properly embedded in $B_{\bar{M}}\left(x_{0}, r_{0}\right)$ for $t \in[0, T]$, where $r_{0}<\left(i_{0} / 2\right), 0<T \leq \varepsilon^{2} r_{0}^{2}$. We assume that at time zero, $x_{0} \in M_{0}$, and the second fundamental form satisfies $|A|(x) \leq r_{0}^{-1}$ on $M_{0} \cap B_{\bar{M}}\left(x_{0}, r_{0}\right)$ and assume $M_{0}$ is graphic in the ball $B_{\bar{M}}\left(x_{0}, r_{0}\right)$. Then we have

$$
|A|(x, t) \leq\left(\varepsilon r_{0}\right)^{-1}
$$

for any $x \in B_{\bar{M}}\left(x_{0}, \varepsilon r_{0}\right) \cap M_{t}, t \in[0, T]$. 
Uniqueness and pseudolocality theorems of the mean curvature flow 485

Proof. By scaling we may assume $r_{0}=1$. By Lemma 7.1, for any $\delta>0$, there is $0<r_{\delta}<1$ such that the connected component of $M_{0} \cap B_{\bar{M}}\left(x_{0},(1 / 96)\right)$ containing $x_{0}$ contains a $\delta$-Lipschitz graph of radius $2 r_{\delta}$ at $x_{0}$. By our graphic assumption, we conclude that $M_{0} \cap B_{\bar{M}}\left(x_{0}, r_{\delta}\right)$ is a $\delta$-Lipschitz graph. So Theorem 7.3 is applicable with radius $r_{\delta}$.

Consequently, for any $\alpha>0$, there exists an $\varepsilon_{\alpha}>0$ such that

$$
|A|(x, t)^{2} \leq \frac{\alpha}{t}+\varepsilon_{\alpha}^{-2}
$$

whenever $x \in M_{t} \cap B_{\bar{M}}\left(x_{0}, \varepsilon_{\alpha}\right), t \in\left[0, \varepsilon_{\alpha}^{2}\right] \cap[0, T]$. Let $\alpha$ be a fixed small constant to be determined later. It turns out that we only need to choose $\alpha=\alpha\left(c_{0}, \bar{n}, n\right)$ finally. Choose $\varepsilon=\min \left\{\sqrt{\alpha} \varepsilon_{\alpha}, 10^{-1}\right\}$. Then by (7.17) we have

$$
|A|(x, t)^{2} \leq \frac{2 \alpha}{t}
$$

whenever $x \in M_{t} \cap B_{\bar{M}}\left(x_{0}, \varepsilon_{\alpha}\right), t \in\left[0, \varepsilon^{2}\right] \cap[0, T]$.

Claim. $|A|(x, t) \leq \varepsilon^{-1}$ holds on $M_{t} \cap B_{\bar{M}}\left(x_{0}, \varepsilon\right), t \in\left[0, \varepsilon^{2}\right] \cap[0, T]$.

Suppose $|A|\left(x_{1}, t_{1}\right)>\varepsilon^{-1}$ holds for some point $\left(x_{1}, t_{1}\right), x_{1} \in M_{t_{1}} \cap$ $B_{\bar{M}}\left(x_{0}, \varepsilon\right), t_{1} \in\left[0, \varepsilon^{2}\right] \cap[0, T]$. We can choose another point $(\bar{x}, \bar{t}), \bar{x} \in M_{\bar{t}} \cap$ $B_{\bar{M}}\left(x_{0}, 4 \varepsilon\right), \bar{t} \in\left[0, \varepsilon^{2}\right] \cap[0, T]$ such that $Q=|A|(\bar{x}, \bar{t}) \geq \varepsilon^{-1}$ and

$$
|A|(x, t) \leq 4 Q
$$

whenever $x \in M_{t}, d_{\bar{M}}(\bar{x}, x) \leq Q^{-1}, 0 \leq t \leq \bar{t}$.

Actually $(\bar{x}, \bar{t})$ can be constructed as the limit of a finite sequence $\left(x_{i}, t_{i}\right)$ satisfying $0 \leq t_{k} \leq t_{k-1}, d_{\bar{M}}\left(x_{0}, x_{k}\right) \leq d_{\bar{M}}\left(x_{0}, x_{k-1}\right)+|A|\left(x_{k-1}, t_{k-1}\right)^{-1},|A|$ $\left(x_{k}, t_{k}\right) \geq 4|A|\left(x_{k-1}, t_{k-1}\right)$. Since

$$
|A|\left(x_{k}, t_{k}\right) \geq 4^{k-1}|A|\left(x_{1}, t_{1}\right) \geq 4^{k-1} \varepsilon^{-1},
$$

$d_{\bar{M}}\left(x_{0}, x_{k}\right) \leq d_{\bar{M}}\left(x_{0}, x_{1}\right)+\sum_{i=1}^{\infty}\left(4^{i-1}|A|\left(x_{1}, t_{1}\right)\right)^{-1} \leq 3 \varepsilon<(1 / 2)$, and the solution is smooth, the sequence must be finite and the last element fits.

Note that $3 n \bar{t} Q^{2} \leq 6 n \alpha \leq(1 / 2)$ by choosing $\alpha \leq(1 / 12 n)$. Let $\psi=(1-$ $\left.\left(d_{\bar{M}}^{2}(\bar{x}, \cdot)+3 n t / Q^{-2}\right)\right)_{+}^{3}$, then we have

$$
\left(\frac{\partial}{\partial t}-\triangle\right) \psi \leq 0
$$


whenever $d_{\bar{M}}(\bar{x}, \cdot)^{2}<\min \left\{\left(1 / c_{0}^{2} e\right), i_{0}^{2}\right\}, t \in[0, \bar{t}]$. On the other hand, by (3.2), the second fundamental form satisfies

$$
\left(\frac{\partial}{\partial t}-\triangle\right)|A|^{2} \leq-|\nabla A|^{2}+C(\bar{n})|A|^{4}+C(\bar{n})\left(1+c_{0}^{2}\right)\left(|A|^{2}+|A|\right) .
$$

Hence

$$
\begin{aligned}
\left(\frac{\partial}{\partial t}-\triangle\right)\left(\psi|A|^{2}\right) \leq & -|\nabla A|^{2} \psi+C(\bar{n})|A|^{4} \psi+C(\bar{n})\left(1+c_{0}^{2}\right)\left(|A|^{2}+|A|\right) \psi \\
& +4|\nabla A||A||\nabla \psi| \\
\leq & C(\bar{n})|A|^{4} \psi+C(\bar{n})\left(1+c_{0}^{2}\right)\left(|A|^{2}+|A|\right) \psi+4 \frac{|\nabla \psi|^{2}}{\psi}|A|^{2} \\
\leq & C(\bar{n})|A|^{4} \psi+C(\bar{n})\left(1+c_{0}^{2}\right)\left(|A|^{2}+|A|\right) \psi \\
& +144 Q^{2}|A|^{2} \psi^{1 / 3}
\end{aligned}
$$

on $[0, \bar{t}]$. By $(7.19)$ and $(7.20)$, we have

$$
\left(\frac{\partial}{\partial t}-\triangle\right)\left(\psi|A|^{2}\right) \leq C(\bar{n}) Q^{4}+C(\bar{n})\left(1+c_{0}^{2}\right)\left(Q+Q^{2}\right) .
$$

From the maximum principle, it follows

$$
\begin{aligned}
\left.\left(\psi|A|^{2}\right)_{\max }\right|_{t=\bar{t}} & \leq 1+C(\bar{n}) Q^{4} \bar{t}+C(\bar{n})\left(1+c_{0}^{2}\right)\left(Q+Q^{2}\right) \bar{t} \\
& \leq 1+2 \alpha C(\bar{n}) Q^{2}+C(\bar{n})\left(1+c_{0}^{2}\right)(\sqrt{2 \alpha \bar{t}}+2 \alpha) .
\end{aligned}
$$

Note that

$$
\left.\left(\psi|A|^{2}\right)_{\max }\right|_{t=\bar{t}} \geq \psi|A|^{2}(\bar{x}, \bar{t}) \geq\left(1-3 n Q^{2} \bar{t}\right)^{3} Q^{2} \geq(1-18 n \alpha) Q^{2},
$$

hence we have

$$
(1-18 n \alpha) Q^{2} \leq 1+2 \alpha C(\bar{n}) Q^{2}+C(\bar{n})\left(1+c_{0}^{2}\right)(\sqrt{2 \alpha \bar{t}}+2 \alpha) .
$$

This implies

$$
Q^{2} \leq \frac{1+C(\bar{n})\left(1+c_{0}^{2}\right)(\sqrt{2 \alpha}+2 \alpha)}{1-(18 n+2 C(\bar{n})) \alpha} .
$$

Choosing suitable small $\alpha=\alpha\left(c_{0}, \bar{n}, n\right)$, we have $Q^{2} \leq 2$, which is a contradiction with $Q^{2}>\varepsilon^{-2}$. So the Claim is proved. 
Uniqueness and pseudolocality theorems of the mean curvature flow 487

We remark that in the above theorem the condition that $M_{0}$ is graphic in the ball $B_{\bar{M}}\left(x_{0}, r_{0}\right)$ can be replaced by any one of the following conditions:

(i) $d_{\bar{g}}(x, y) \geq\left(d_{g_{0}}(x, y) / C\right)$ for any $x, y \in M_{0} \cap B_{\bar{M}}\left(x_{0}, r_{0}\right)$;

(ii) there is a $\epsilon_{0}>0$ such that $B_{\bar{M}}\left(x_{0}, \epsilon r_{0}\right) \cap M_{0}$ is connected for any $\epsilon \leq \epsilon_{0}$.

Corollary 7.6. Let $\bar{M}$ be an $\bar{n}$-dimensional complete manifold satisfying $\sum_{i=0}^{3}\left|\bar{\nabla}^{i} \overline{\mathrm{R}} \mathrm{m}\right| \leq c_{0}^{2} \quad$ and $\operatorname{inj}(\bar{M}) \geq i_{0}>0$. Let $X_{0}: M \rightarrow \bar{M}$ be an $n$-dimensional isometrically properly embedded submanifold with bounded second fundamental form $|A| \leq c_{0}$ in $\bar{M}$. We assume $M_{0}=X_{0}(M)$ is uniform graphic with some radius $r>0$. Suppose $X(x, t)$ is a smooth solution to the mean curvature flow (1.1) on $M \times\left[0, T_{0}\right]$ properly embedded in $\bar{M}$ with $X_{0}$ as initial data. Then there is $T_{1}>0$ depending upon $c_{0}, i_{0}, r$ and the dimension $\bar{n}$ such that

$$
|A|(x, t) \leq 2 c_{0}
$$

for all $x \in M, 0 \leq t \leq \min \left\{T_{0}, T_{1}\right\}$.

Proof. By Theorem 7.5, there is $\epsilon>0$ such that for any $x_{0} \in M$, we have

$$
|A|(x, t) \leq \epsilon^{-1}
$$

on $B_{\bar{M}}\left(x_{0}, \epsilon\right), t \in\left[0, \epsilon^{2}\right] \cap[0, T]$. Let $[0, \gamma) \subset\left[0, \epsilon^{2}\right] \cap[0, T]$ be the maximal time interval so that the orbit of $x_{0}, x_{0}^{t} \in B_{\bar{M}}\left(x_{0}, \epsilon\right)$ for $t \in[0, \gamma]$. Then by the MCF equation, we know

$$
\frac{d}{d t} d_{\bar{M}}\left(x_{0}, x_{0}^{t}\right) \leq C \epsilon^{-1},
$$

for any $t \in[0, \gamma]$. This implies $\gamma \geq\left(\epsilon^{2} / C\right)$ for some $C=C(n, \bar{n})$. Choosing $\varepsilon=(\epsilon / \sqrt{C}), T=\min \left\{T_{0}, \varepsilon^{2}\right\}$, we conclude that the second fundamental forms are uniformly bounded by the constant $\varepsilon^{-1}$ on $M \times[0, T]$. Once the second fundamental form is bounded, since we assumed $\sum_{i=0}^{3}\left|\nabla^{i} \overline{\mathrm{R}} \mathrm{m}\right| \leq c_{0}^{2}$, we have gradient estimate $|\nabla A| \leq(C / \sqrt{t})$, and hence suitable linear growth function with bounded first and second derivatives can be constructed. Therefore we can apply the maximum principle to the equation of $|A|$ to conclude a uniform estimate $|A| \leq 2 c_{0}$, for any $t \in\left[0,\left(1 / C(\bar{n}) c_{0}^{2}\right)\right]$. Set $T_{1}=\min \left\{T,\left(1 / C(\bar{n}) c_{0}^{2}\right)\right\}$. The proof is completed.

Theorem 1.3 follows as a corollary of Theorem 1.1 and Corollary 7.6. 
In [16], Huisken established his monotonicity formula, which was later generalized by Ecker-Huisken [11], Huisken [17], and to parabolic flows on Riemannian manifolds by Hamilton [15]. The remarkable localized monotonicity formula was discovered by Ecker [9].

Finally, we are acknowledged kindly by Prof. Klaus Ecker that part of our arguments of using monotonicity was also carried out before by B. White [25] (see [10]) in different situations. The choice of $\psi$ in inequality (7.6) appeared first in [1] for the case of Euclidean space, see Remark 4.8 of the book [10].

\section{Acknowledgment}

We are grateful to Professor Xi-Ping Zhu for useful conversations and encouragement. We are also thankful for beneficial comments from Professor Klaus Ecker who brought our attention to some references. The second author would like to thank Professor Nai-Chung Leung and Professor Luen-Fai Tam for their constant teaching and encouragement, and Professor Mu-Tao Wang for very helpful discussions. The first author is partially supported by Grants 2005-34000-3171404 and 2006-34000-1131040.

\section{References}

[1] K. A. Brakke, The motion of a surface by its mean curvature, Mathematical Notes, vol. 20, Princeton University Press, Princeton, NJ, 1978.

[2] H. D. Cao and X. P. Zhu, A complete proof of the Poincaré and geometrization conjectures - application of the Hamilton-Perelman theory of the Ricci flow, Asian J. Math. 10 (2006), no. 2, 165-492.

[3] J. Cheeger and D. Ebin, Comparison theorems in Riemannian geometry, North-Holland, Amsterdam, 1975.

[4] B. L. Chen and X. P. Zhu, Ricci flow with surgery on four manifolds with positive isotropic curvature, J. Diff. Geom. 74 (2006), 177-264.

[5] B. L. Chen and X. P. Zhu, Uniqueness of the Ricci flow on complete noncompact manifolds, J. Diff. Geom. 74 (2006), 119-154.

[6] K. S. Chou and X. P. Zhu, Shortening complete plane curves, J. Diff. Geom. 50 (1998), 471-504. 
Uniqueness and pseudolocality theorems of the mean curvature flow 489

[7] D. De Turck, Deforming metrics in the direction of their Ricci tensors, J. Diff. Geom. 18 (1983), 157-162.

[8] W. Y. Ding and F. H. Lin, A generalization of Eells-Sampson's theorem, J. Partial Diff. Equations 5 (1992), no. 4, 13-22.

[9] K. Ecker, A local monotonicity formula for mean curvature flow, Ann. Math. 154 (2001), 503-525.

[10] K. Ecker, Regularity theory for mean curvature flow, Progress in Nonlinear Differential Equations and their Applications, vol. 57, Birkäuser Boston, Inc., Boston, MA, 2004.

[11] K. Ecker and G. Huisken, Mean curvature evolution of entire graphs, Ann. Math. 130 (1989), 453-471.

[12] K. Ecker and G. Huisken, Interior estimates for hypersurfaces moving by mean curvature, Invent. Math. 105 (1991), 547-569.

[13] J. Eells and J. Sampson, Harmonic mappings of Riemannian manifolds, Amer. J. Math. 86 (1964), 109-160.

[14] R. S. Hamilton, A compactness property for solution of the Ricci flow, Amer. J. Math. 117 (1995), 545-572.

[15] R. S. Hamilton, Monotonicity formulas for parabolic flows on manifolds, Comm. Anal. Geom. 1 (1993), 127-137.

[16] G. Huisken, Asymptotic behaviour for singularities of the mean curvature flow, J. Diff. Geom. 31 (1990), 285-299.

[17] G. Huisken, Local and global behaviour of hypersurfaces moving by mean curvature flow, Proceedings of the Centre for Mathematics and its Applications, vol. 26, Australian National University, 1991.

[18] H. B. Lawson, Jr. and R. Osserman, Non existence, non uniquness and irregularity of solutions to minimal surface system, Acta Math. 139 (1977), nos 1-2 1-17.

[19] O. A. Ladyzenskaja, V. A. Solonnikov and N. N. Uralceva, Linear and quasilinear equations of parabolic type, Translations of Mathematical Monographs, vol. 23, AMS, Providence, R.I., 1967, xi +648 pp.

[20] G. Perelman, The entropy formula for the Ricci flow and its geometric applications, Preprint, arXiv:math. DG/0211159 v1. 
[21] G. Perelman, Ricci flow with surgery on three manifolds, Preprint, 2003 arXiv:math. DG/0303109 v1.

[22] R. Schoen and S. T. Yau, Lectures on differential geometry, Conference proceedings and Lecture Notes in Geometry and Topology, vol. 1, International Press Publications, 1994.

[23] R. Schoen and S. T. Yau, Module space of harmonic maps, compact group actions and the topology of manifolds with non-positive curvature, in 'Lectures on harmonic maps', International Press, 1997.

[24] M .T. Wang, The mean curvature flow smoothes Lipschitz submanifolds, Comm. Anal. Geom. 12 (2004), no 3, 581-599.

[25] B. White, A local regularity theorem for classical mean curvature flow, Preprint 2000.

Department of Mathematics

Sun YAT-SEN UNIVERSITY

GuANGZHOU 510275

P.R. China

E-mail address: mcscbl@mail.sysu.edu.cn

Institute of Mathematical Sciences and Department of Mathematics The Chinese University of Hong Kong

HONG KONG

P.R. ChinA

E-mail address: lyin@math.cuhk.edu.hk

Received February 20, 2006 